quatrième série-tome 41 fascicule 2 mars-avril 2008

$$
\begin{aligned}
& \text { ANNALES } \\
& \text { SCIENTIFIQUES } \\
& \text { de } \\
& \text { L'ECOLE } \\
& \text { NORMALE } \\
& \text { SUPÉRIEURE }
\end{aligned}
$$

Georges COMTE \& Michel MERLE

$$
\text { Équisingularité réelle II : }
$$

invariants locaux et conditions de régularité 


\title{
ÉQUISINGULARITÉ RÉELLE II : INVARIANTS LOCAUX ET CONDITIONS DE RÉGULARITÉ
}

\author{
PAR Georges COMTE et Michel MERLE \\ (Appendice de G. Comte, P. Graftieaux \& M. Merle)
}

RÉSumÉ. - On définit, pour un germe d'ensemble sous-analytique, deux nouvelles suites finies d'invariants numériques. La première a pour termes les localisations des courbures de Lipschitz-Killing classiques, la seconde est l'équivalent réel des caractéristiques évanescentes complexes introduites par M. Kashiwara. On montre que chaque terme d'une de ces suites est combinaison linéaire des termes de l'autre, puis on relie ces invariants à la géométrie des discriminants des projections du germe sur des plans de toutes les dimensions. Il apparaît alors que ces invariants sont continus le long de strates de Verdier d'une stratification sous-analytique d'un fermé.

Abstract. - For germs of subanalytic sets, we define two finite sequences of new numerical invariants. The first one is obtained by localizing the classical Lipschitz-Killing curvatures, the second one is the real analogue of the vanishing Euler characteristics introduced by M. Kashiwara. We show that each invariant of one sequence is a linear combination of the invariants of the other sequence. We then connect our invariants to the geometry of the discriminants of all dimension. Finally we prove that these invariants are continuous along Verdier strata of a closed subanalytic set.

\section{Introduction}

Désignons par $X$ un ensemble sous-analytique de $\mathbb{R}^{n}$ et pour $y \in X$, notons $X_{y}$ le germe de $X$ en $y$ puis $d$ sa dimension.

Dans [11] il est prouvé que la fonction densité locale $y \mapsto \Theta_{d}\left(X_{y}\right)$ (la localisation du volume) est une fonction continue le long de strates de Verdier ou même $\left(b^{*}\right)$-régulières de $X$ (voir [81], [82] pour une preuve du caractère lipschitzien de la densité le long de strates de Verdier et pour la continuité le long de strates de Whitney). Il s'agissait dans [11] de ramener l'étude de la densité à celle d'un invariant $\sigma_{d}\left(X_{y}\right)$ associé aux discriminants des projections du germe $X_{y}$ sur des plans de dimension $d$, via une formule du type Cauchy-Crofton localisée égalant $\Theta_{d}\left(X_{y}\right)$ et $\sigma_{d}\left(X_{y}\right)$ ([10], [11, Théorèmes 1.10 et 1.16]). Ce type de résultats 
reliant le comportement d'un invariant et la géométrie des discriminants s'inscrit dans le programme de l'équisingularité inauguré par Zariski pour les ensembles algébriques complexes (voir [85], [86], [87], [88], [89], [90]). La condition géométrique portant sur les discrimants $(d-1)$-dimensionnels qui assure la continuité de la densité le long d'une strate $Y$ est que ceux-ci aient le long de $Y$ un cône normal dont la dimension des fibres soit majorée par la dimension générique, i.e. $d-\operatorname{dim}(Y)-1$. Il est prouvé dans [11, Proposition 3.7], que cette condition est satisfaite le long de strates de Verdier. Dans le cadre analytique complexe densité locale et multiplicité coïncident ([13]), de même que conditions de Whitney et de Verdier ([31], [74]). Ce faisant, le résultat de [11] étend à la géométrie réelle celui de [34] selon lequel la condition de Whitney assure l'équimultiplicité.

Dans le présent article, nous étendons l'étude faite dans [11] à toutes les dimensions de projection : nous présentons ici des invariants polaires et de courbures dont le comportement traduit la géométrie des discriminants de $X_{y}$ associés à des projections sur des plans de dimension $i$, pour $0 \leq i \leq d$. Pour cela, d'une part nous considérons le volume comme le dernier invariant de la suite :

$$
\Lambda_{*}(X)=\left(\Lambda_{0}(X)=\chi(X), \cdots, \Lambda_{d}(X)\right)
$$

des courbures de Lipschitz-Killing de $X$, puisque l'égalité $\Lambda_{d}(X)=\operatorname{Vol}_{d}(X)$ est la formule de Cauchy-Crofton classique. La localisation en $y$ de $\Lambda_{*}(X)$ permet alors de définir une suite de nouveaux invariants attachés au germe $X_{y}$ :

$$
\Lambda_{*}^{\mathrm{loc}}\left(X_{y}\right)=\left(\Lambda_{0}^{\mathrm{loc}}\left(X_{y}\right)=1, \ldots, \Lambda_{d}^{\mathrm{loc}}\left(X_{y}\right)=\Theta_{d}\left(X_{y}\right)\right) .
$$

Nous appelons ces invariants les courbures de Lipschitz-Killing locales. D'autre part la définition de l'invariant $\sigma_{d}\left(X_{y}\right)$ se généralise à toutes les dimensions, ce qui donne lieu à la suite des invariants polaires:

$$
\sigma_{*}\left(X_{y}\right)=\left(\sigma_{0}\left(X_{y}\right)=1, \cdots, \sigma_{d}\left(X_{y}\right)\right) .
$$

L'égalité $\sigma_{d}\left(X_{y}\right)=\Lambda_{d}^{\text {loc }}\left(X_{y}\right)\left(=\Theta_{d}\left(X_{y}\right)\right)$ est la formule de Cauchy-Crofton locale de [11]. Celle-ci pose la question de la dépendance des termes d'une des deux suites $\Lambda_{*}^{\text {loc }}$ et $\sigma_{*}$ en fonction des termes de l'autre. Cette possibilité est notamment appuyée par le problème posé dans [27] (Problème 14 ou [9, 14.3]) consistant à se demander si le théorème de Hadwiger possède un analogue en géométrie convexe sphérique. En effet, si pour un convexe $K$ de la sphère unité $S^{n-1}$ on note $\widehat{K}$ le cône de sommet 0 supporté par $K$, les suites $\sigma_{*}$ et $\Lambda_{*}^{\text {loc }}$ définissent les valuations $K \mapsto \sigma_{*}\left(\widehat{K}_{0}\right)$ et $K \mapsto \Lambda_{*}^{\text {loc }}\left(\widehat{K}_{0}\right)$ sur les convexes sphériques. Celles-ci sont continues relativement à la métrique de Hausdorff et invariantes sous les rotations de la sphère. Dans le cas euclidien il est connu depuis [28] (voir aussi [39]) que les valuations sur les convexes de $\mathbb{R}^{n}$ continues et invariantes sous les isométries forment un espace vectoriel de dimension $n+1$ dont une base est la famille $\Lambda_{*}$. Le problème toujours en suspens (résolu seulement pour $n \leq 3$ ) posé dans [27] est celui de la validité (d'une formulation équivalente) de ce théorème de finitude dans le cas sphérique. La solution positive de ce problème aurait pour conséquence immédiate que les invariants de la suite $\Lambda_{*}^{\text {loc }}$ sont des combinaisons linéaires de ceux de $\sigma_{*}$. 
Dans cette direction, pour $X_{y}$ un germe d'ensemble sous-analytique quelconque, nous montrons que chaque élément d'une des suites de $\Lambda_{*}^{\text {loc }}\left(X_{y}\right)$ ou $\sigma_{*}\left(X_{y}\right)$ est en effet combinaison linéaire (à coefficients universels) des éléments de l'autre. Plus précisément (Théorème 3.1) il existe une matrice triangulaire supérieure $\mathcal{M}$ n'ayant que des 1 sur sa diagonale, telle que :

$$
\Lambda_{*}^{\mathrm{loc}}=\mathcal{M} \cdot \sigma_{*} .
$$

Puisque la dernière ligne de cette égalité matricielle redonne la formule de Cauchy-Crofton locale $\Theta_{d}=\sigma_{d}$, on peut voir $\Lambda_{*}^{\text {loc }}=\mathcal{M} \cdot \sigma_{*}$ comme une formule de Cauchy-Crofton locale multi-dimensionnelle.

Pour une présentation plus large des questions liées aux valuations définies sur les convexes ainsi que pour le calcul des coefficients de la matrice $\mathcal{M}$, nous renvoyons le lecteur à l'appendice.

En tant que moyenne, sur les projections génériques, d'intégrales (relativement à la densité locale) de fonctions constructibles sur les projetés de $X_{y}$, les termes de la suite $\sigma_{*}\left(X_{y}\right)$ sont les analogues réels des caractéristiques évanescentes introduites dans le cadre analytique complexe par M. Kashiwara dans [37] et étudiées dans [16], [17], [8], [76], [77], [78] (voir aussi [56]). Dans le cas des hypersurfaces à singularité isolée il s'agit (à coefficients près) de la suite $\mu_{*}$ des nombres de Milnor des sections planes de $X_{y}$. Il est montré dans [5] que pour une famille analytique d'hypersurfaces analytiques complexes $\left(X_{y}\right)_{y \in \mathbb{C}}$ à singularité isolée et vérifiant la condition de Whitney le long de l'axe des paramètres $y$, la suite $\mu_{*}$ est constante. Dans le cas général, et non plus seulement dans celui des hypersurfaces, d'après [17], [76], [78], chaque terme de la suite $\widetilde{\sigma}_{*}\left(X_{y}\right)$ des invariants polaires complexes est combinaison linéaire des éléments de la suite $\widetilde{m}_{*}\left(X_{y}\right)$ des multiplicités des variétés polaires de $X_{y}$. Ces dernières étant constantes le long de strates de Whitney ([31], [58], [74]), la suite $\widetilde{\sigma}_{*}\left(X_{y}\right)$ est elle-même constante le long de strates de Whitney d'un ensemble analytique complexe.

Nous donnons ici la version réelle de ce résultat (Théorèmes 4.9 et 4.10), en montrant que le long d'une strate de Verdier $Y$ d'un ensemble sous-analytique fermé $X$, l'application $Y \ni y \mapsto \sigma_{*}\left(X_{y}\right)$ est continue, généralisant à toutes les dimensions la continuité, prouvée dans [11], de la seule fonction $Y \ni y \mapsto \sigma_{d}\left(X_{y}\right)$. En particulier, chaque courbure localisée $\Lambda_{i}^{\text {loc }}$ étant combinaison linéaire des invariants $\sigma_{j}$, nous en déduisons la continuité de $y \mapsto \Lambda_{*}^{\text {loc }}\left(X_{y}\right)$ le long des strates $Y$ d'une stratification de Verdier de $X$.

Notons que bien qu'en géométrie complexe il y ait équivalence entre la constance des caractéristiques évanescentes le long d'une strate et le fait que celle-ci soit une strate d'une stratification de Whitney, on ne peut espérer une telle réciproque en géométrie réelle. Par exemple dans $\mathbb{R}^{3}$ si $Y$ est l'axe $O y$ et si $X$ est le semi-algébrique suivant (une fronce le long de l'axe $O y$, pincée sur l'axe $O z$ ) :

$$
X=\left\{(x, y, z) \in \mathbb{R}^{3} ; y z^{3}=x^{3}-3 z^{3} x, z \geq 0\right\},
$$

avec les notations de la définition 2.7 on vérifie que :

- $\forall y \in Y, \sigma_{1}\left(X_{y}\right)=1$. Ceci est clair pour $y \neq 0$. Mais pour $y=0$ on observe, suivant les notations du théorème 2.6, que pour des projections génériques sur des droites $P$ de $\mathbb{R}^{3}$ (celles telles que $O z \not \subset P^{\perp}$ ), $n_{P}=2, \chi_{1}^{P}=\chi_{2}^{P}=1$ et $\Theta_{1}\left(\mathcal{K}_{1}^{P}\right)=\Theta_{1}\left(\mathcal{K}_{2}^{P}\right)=1 / 2$. C'est-à-dire que $\sigma_{1}\left(X_{0}\right)=1$. 
- $\forall y \in Y, \sigma_{2}\left(X_{y}\right)=\Theta_{2}\left(X_{y}\right)=1 / 2$. Encore une fois ceci est clair pour $y \neq 0$, puisque dans ce cas $X_{y}$ est une hypersurface à bord de bord $O y$. Pour $y=0$, on calcule $\Theta_{2}\left(X_{0}\right)$ en calculant la 2-densité des composantes du cône tangent pur de $X_{0}$ affectés de leur multiplicité. On obtient $\Theta_{2}\left(X_{0}\right)=1 .(1 / 4)+1 .(1 / 4)=1 / 2$ (cf. [44]).

Les suites $\Lambda_{*}^{\text {loc }}$ et $\sigma_{*}$ sont par conséquent continues (et même constantes) le long de $Y$, sans pour autant que $X^{\text {rég }}$ soit $(w)$ ou $(b)$-régulier le long de $Y$, puisque pas même $(a)$-régulier. Notons de plus que sur cet exemple les discriminants généraux (de dimension 1 ) ne satisfont pas la condition géométrique du théorème 4.9 , dont on montre qu'elle suffit à la continuité de nos invariants locaux.

Contenu. - Cet article s'organise de la façon suivante :

Dans la section 1, nous montrons comment les courbures de Lipschitz-Killing $\Lambda_{i}(X)$ d'un ensemble $X$ définissable dans une structure o-minimale sur les réels se localisent en des invariants $\Lambda_{i}^{\text {loc }}\left(X_{x}\right)$ attachés au germe de $X$ en $x$. Nous traitons explicitement le cas des ensembles sous-analytiques à l'aide du théorème d'isotopie de Thom-Mather, ce qui n'est pas restrictif puisque les ensembles définissables dans une structure o-minimale sur $(\mathbb{R},+,$.$) ad-$ mettent des stratifications de Whitney ([52], [70] par exemple), mais on pourrait aussi bien invoquer le théorème de décomposition cellulaire ([14], [62], [40]), dont les conséquences en termes de finitude uniforme suffisent pour nos preuves.

Dans la section 2, nous définissons les invariants polaires $\sigma_{i}\left(X_{x}\right)$ (Section 2.1). Il s'agit essentiellement de remarquer que les directions de projection générales ne rencontrent pas les variétés polaires du germe $X_{x}$ qu'elles définissent (Proposition 2.2), pas plus que les lieux critiques du link de $X_{x}$ associés à ces projections (Proposition 2.5). Nous interprétons ensuite (Section 2.2) les invariants $\sigma_{i}$ dans le cas analytique complexe et nous rappelons que leur constance le long des strates d'une stratification équivaut à la $(b)$-régularité de celle-ci (Théorème 2.10).

Dans la section 3, nous montrons le théorème 3.1 évoqué en introduction, c'est-à-dire l'existence d'une matrice triangulaire $\mathcal{M}$ telle que $: \Lambda_{*}^{\text {loc }}=\mathcal{M} \cdot \sigma_{*}$. Nous commençons par montrer cette égalité pour les ensembles définissables (sous-analytiques dans le texte) coniques (Section 3.1) en utilisant les techniques de calcul de [6]. Nous montrons que dans le cas conique $\Lambda_{i}^{\text {loc }}\left(X_{x}\right)$ et $\sigma_{i}\left(X_{x}\right)$ sont des combinaisons linéaires des courbures de LipschitzKilling $\Lambda_{j}(L)$ du link $L=X \cap S_{(x, 1)}$ de $X$. Le cas général (Section 3.2) s'en déduit par déformation transverse sur le cône tangent.

Dans la section 4, nous établissons un lien entre la variation de $\Lambda_{*}^{\text {loc }}$ et $\sigma_{*}$ le long d'une strate d'une stratification de $X$ et la régularité de cette stratification : la $(w)$-régularité assure la continuité des invariants polaires. Pour cela nous montrons dans un premier temps comment la condition $\left(b^{*}\right)$ permet de calculer $\sigma_{i}\left(X_{x}\right)$, pour $x$ au voisinage de 0 et le long d'une strate, à l'aide, pour chaque direction de projection générique, d'un bon représentant du seul germe $X_{0}$ en 0 (Proposition 4.4). Au passage, l'existence de tels bons voisinages assure immédiatement dans le cas complexe la constance des caractéristiques évanescentes le long de strates de Whitney (Corollaire 4.5) et ce sans mentionner la constance des multiplicités des variétés polaires et la formule qui les relie aux caractéristiques évanescentes (voir [17], [78]). Dans un second temps, et en s'appuyant sur l'existence de bons voisinages, nous montrons qu'une condition suffisant à la continuité des invariants polaires réels $\sigma_{i}$ porte sur le contrôle 
de la dimension des fibres des cônes normaux aux discriminants (Théorème 4.9) : il s'agit de la version géométrique de l'équimultiplicité des variétés polaires et des discriminants, encore pertinente en géométrie réelle, et le théorème 4.10 montre que cette condition est en géométrie réelle une conséquence de la $(w)$-régularité, comme c'est déjà le cas en complexe.

Notations. - Nous noterons $B_{(x, r)}$ ou au besoin $B_{(x, r)}^{n}$ la boule fermée de centre $x$ et de rayon $r$ dans $\mathbb{R}^{n}, S_{(x, r)}^{n-1}$ sa frontière, $\mathcal{H}^{i}$ la mesure $i$-dimensionnelle de Hausdorff, $\alpha_{i}=\mathcal{H}^{i}\left(B_{(0,1)}^{i}\right)$, avec la convention $\alpha_{0}=1, C_{i}^{j}=\frac{j !}{(j-i) ! i !}$ pour deux entiers $0 \leq i \leq j$, $\chi$ la caractéristique d'Euler-Poincaré, $1_{E}$ la fonction caractéristique d'un sous-ensemble $E$ de $\mathbb{R}^{n}, e(X, x)$ la multiplicité en $x$ d'un ensemble analytique complexe $X, \mathcal{O}_{n}(\mathbb{R})$ le groupe orthogonal de $\mathbb{R}^{n}, U_{n}(\mathbb{C})$ le groupe unitaire de $\mathbb{C}^{n}, G(i, n)$ la grassmannienne des $i$-plans vectoriels de $\mathbb{R}^{n}, \bar{G}(i, n)$ la grassmannienne des $i$-plans affines de $\mathbb{R}^{n}, \widetilde{G}(i, n)$ la grassmannienne des $i$-plans vectoriels (complexes) de $\mathbb{C}^{n}, \gamma_{i, n}$ la mesure unitaire naturelle sur $G(i, n)$, $\bar{\gamma}_{i, n}$ la mesure naturelle sur $\bar{G}(i, n)$, et pour $P$ un $i$-plan de $\mathbb{R}^{n}$ ou $\mathbb{C}^{n}, \pi_{P}$ la projection orthogonale sur $P$.

\section{Invariants de Lipschitz-Killing locaux}

Dans cette partie nous montrons comment les propriétés de finitude uniforme locale des ensembles sous-analytiques (ou plus largement des ensembles définissables dans une structure o-minimale [15], [70]) permettent de localiser les courbures de Lipschitz-Killing. Nous mentionnons [2, Section 5], pour une notion comparable de localisation des courbures de Lipschitz-Killing : les localisations de [2] sont des combinaisons linéaires des nôtres, comme cela est indiqué dans [2]. Nous rappelons auparavant les définitions et propriétés essentielles de ces courbures. Sur ce sujet nous renvoyons sans plus le répéter dans la suite, entre autres références à [1], [2], [3], [6], [9], [19], [21], [22], [24], [23], [25], [48], [47], [45], [55], [69], [68], [72], [71], [84].

Considérons $X$ un ensemble sous-analytique compact (dans toute cette section) de $\mathbb{R}^{n}$ et notons, pour $r$ un réel $>0, \mathcal{T}_{r}(X)$ le voisinage tubulaire de rayon $r$ de $X$, c'est-à-dire :

$$
\mathcal{T}_{r}(X)=\bigcup_{x \in X} B_{(x, r)} .
$$

Nous définissons alors la quantité $\mathcal{V}_{X}(r)$ de la façon suivante :

$$
\mathcal{V}_{X}(r)=\int_{x \in \mathcal{T}_{r}(X)} \chi\left(X \cap B_{(x, r)}\right) d \mathcal{H}^{n}(x) .
$$

Bien sûr, lorsque $X$ est lisse et pour $r$ suffisamment petit :

$$
\mathcal{V}_{X}(r)=\int_{x \in \mathcal{T}_{r}(X)} d \mathcal{H}^{n}(x)=\mathcal{H}^{n}\left(\mathcal{T}_{r}(X)\right)
$$

On appelle $\mathcal{V}_{X}(r)$ le volume modifié de $\mathcal{T}_{r}(X)$. D'après [23], [2], [6], quel que soit $r \geq 0, \mathcal{V}_{X}(r)$ est un polynôme de degré $n$ en la variable $r$, que nous notons :

$$
\mathcal{V}_{X}(r)=\Lambda_{n}(X)+\Lambda_{n-1}(X) \cdot \alpha_{1} \cdot r+\cdots+\Lambda_{1}(X) \cdot \alpha_{n-1} \cdot r^{n-1}+\Lambda_{0}(X) \cdot \alpha_{n} \cdot r^{n} .
$$

On dispose de plus d'une formule de représentation intégrale pour chaque $\Lambda_{i}(X)$ : 
ThÉORÈme 1.1. - Soit $X$ un ensemble sous-analytique compact de $\mathbb{R}^{n}$. Pour tout $i \in\{0, \ldots, n\}$, on a l'égalité :

$$
\Lambda_{i}(X)=\int_{\bar{P} \in \bar{G}(n-i, n)} \chi(X \cap \bar{P}) \frac{d \bar{\gamma}_{n-i, n}(\bar{P})}{\beta(n, i)},
$$

où $\beta(n, i)$ est la constante universelle $\Gamma\left(\frac{n-i+1}{2}\right) \Gamma\left(\frac{i+1}{2}\right) / \Gamma\left(\frac{n+1}{2}\right) \Gamma\left(\frac{1}{2}\right)$ et $\Gamma$ est la fonction d'Euler.

DéFInItion 1.2. - Les quantités $\Lambda_{i}(X)$ sont appelées les courbures de Lipschitz-Killing de $X$.

Supposons que l'origine de $\mathbb{R}^{n}$ soit dans $X$, et notons $X_{0}$ le germe à l'origine que définit $X$. Nous allons montrer dans cette section (Théorème 1.3) que l'on peut localiser les courbures

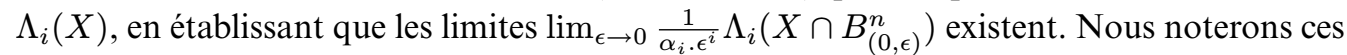
limites $\Lambda_{i}^{\text {loc }}\left(X_{0}\right)$, et nous les appellerons les invariants de Lipschitz-Killing locaux, ou les courbures de Lipschitz-Killing locales du germe $X_{0}$. Pour certaines valeurs de $i$ et de $d$, l'existence des limites $\Lambda_{i}^{\text {loc }}$ est claire. En effet, lorsque :

- $i=0: \Lambda_{i}\left(X \cap B_{(0, \epsilon)}^{n}\right)=\chi\left(X \cap B_{(0, \epsilon)}^{n}\right)=1$, pour $\epsilon$ suffisamment petit, du fait de la structure conique de $X_{0}$. De sorte que l'on peut poser $\Lambda_{0}^{\text {loc }}\left(X_{0}\right)=1$.

- $i>\operatorname{dim}\left(X_{0}\right):$ l'intersection $X \cap \bar{P}$ est génériquement vide, pour $\bar{P} \in \bar{G}(n-i, n)$, ce qui donne : $\Lambda_{i}\left(X \cap B_{(0, \epsilon)}^{n}\right)=0$, et donc $\Lambda_{i}^{\text {loc }}\left(X_{0}\right)=0$.

- $i=d=\operatorname{dim}\left(X_{0}\right)$ : l'intersection $X \cap \bar{P}$ est, pour $\bar{P} \in \bar{G}(n-d, n)$ général, un nombre fini de points $N(\bar{P}, X)=N(P, X, y)$, lorsque $\bar{P}=\pi_{P}^{-1}(y)$. On a alors :

$$
\begin{aligned}
\Lambda_{d}\left(X \cap B_{(0, \epsilon)}^{n}\right) & =\int_{\bar{P} \in \bar{G}(n-d, n)} N\left(\bar{P}, X \cap B_{(0, \epsilon)}^{n}\right) \frac{d \bar{\gamma}_{n-d, n}(\bar{P})}{\beta(n, d)} \\
& =\int_{P \in G(d, n)} \int_{y \in P} N\left(P, X \cap B_{(0, \epsilon)}^{n}, y\right) d \mathcal{H}^{d}(y) \frac{d \gamma_{d, n}(P)}{\beta(n, d)},
\end{aligned}
$$

ce qui donne, d'après la formule classique de Cauchy-Crofton pour le volume ([18, 5.11], [20, 2.10.15], [64, 14.69]) :

$$
\begin{gathered}
\Lambda_{d}\left(X \cap B_{(0, \epsilon)}^{n}\right)=\mathcal{H}^{d}\left(X \cap B_{(0, \epsilon)}^{n}\right) \text { et donc } \\
\lim _{\epsilon \rightarrow 0} \frac{1}{\alpha_{d} \cdot \epsilon^{d}} \Lambda_{d}\left(X \cap B_{(0, \epsilon)}^{n}\right)=\lim _{\epsilon \rightarrow 0} \frac{1}{\alpha_{d} \cdot \epsilon^{d}} \mathcal{H}^{d}\left(X \cap B_{(0, \epsilon)}^{n}\right) .
\end{gathered}
$$

Or d'après le théorème de Kurdyka et Raby ([44], [43]), qui sera par conséquent obtenu comme corollaire du théorème 1.3 (Corollaire 1.4), cette limite existe bien, il s'agit de la $d$-densité du germe $X_{0}$ (cf. [20], [49] pour le cas analytique complexe). On la note traditionnellement :

$$
\Theta_{d}\left(X_{0}\right)=\lim _{\epsilon \rightarrow 0} \frac{1}{\alpha_{d} \cdot \epsilon^{d}} \mathcal{H}^{d}\left(X \cap B_{(0, \epsilon)}^{n}\right) .
$$

En résumé, dans le théorème 1.3, en localisant les courbures de Lipschitz-Killing de $X$, nous définissons une suite finie d'invariants du germe $X_{0}$ :

$$
\left(\Lambda_{0}^{\text {loc }}\left(X_{0}\right)=1, \Lambda_{1}^{\text {loc }}\left(X_{0}\right), \ldots, \Lambda_{d}^{\text {loc }}\left(X_{0}\right)=\Theta_{d}\left(X_{0}\right), 0, \ldots, 0\right),
$$

dont le terme de rang $d$ est la $d$-densité de $X_{0}$, c'est-à-dire la localisation habituelle du volume $d$-dimensionnel $\Lambda_{d}=\mathcal{H}^{d}$. 
ThÉORÈme 1.3. - Soit $X$ un ensemble sous-analytique compact de $\mathbb{R}^{n}$, représentant quelconque du germe $X_{0}$. Avec les notations précédentes, quel que soit $i \in\{0, \ldots, n\}$, la limite suivante existe :

$$
\lim _{\epsilon \rightarrow 0} \frac{1}{\alpha_{i} \cdot \epsilon^{i}} \Lambda_{i}\left(X \cap B_{(0, \epsilon)}^{n}\right) .
$$

Nous notons ces limites $\left(\Lambda_{i}^{\mathrm{loc}}\left(X_{0}\right)\right)_{i \in\{0, \ldots, n\}}$ et nous les appelons les invariants de LipschitzKilling locaux ou les courbures de Lipschitz-Killing locales du germe $X_{0}$. De plus :

$$
\Lambda_{0}^{\text {loc }}\left(X_{0}\right)=1, \Lambda_{d}^{\text {loc }}\left(X_{0}\right)=\Theta_{d}\left(X_{0}\right), \text { pour } d=\operatorname{dim}\left(X_{0}\right) \text { et } \Lambda_{i}^{\text {loc }}\left(X_{0}\right)=0, \text { pour } i>d \text {. }
$$

Démonstration. - Soit $i \in\{0, \ldots, n\}$. Commençons par déformer $X$ sur son cône tangent dans un produit. Notons :

$$
X_{\epsilon}=\frac{1}{\epsilon} .\left(X \cap B_{(0, \epsilon)}^{n}\right) \subset B_{(0,1)}^{n} \text { et }[\bar{G}(n-i, n)]_{1}=\left\{\bar{P} \in \bar{G}(n-i, n) ; \bar{P} \cap B_{(0,1)}^{n} \neq \varnothing\right\} .
$$

Le sous-analytique $[\bar{G}(n-i, n)]_{1}$ de $\bar{G}(n-i, n)$ est compact. Considérons maintenant les sous-analytiques suivants de $G=[\bar{G}(n-i, n)]_{1} \times[0,1] \times B_{(0,1)}^{n-i}$ (on identifie $\bar{P} \cap B^{n}(0,1)$ et $\left.B^{n-i}(0,1)\right)$ :

$$
\begin{aligned}
& \left.\left.E=\left\{(\bar{P}, \epsilon, x) ; \bar{P} \in[\bar{G}(n-i, n)]_{1}, \epsilon \in\right] 0,1\right], x \in X_{\epsilon} \cap \bar{P}\right\} \\
& \left.\left.F=\left\{(\bar{P}, \epsilon, x) ; \bar{P} \in[\bar{G}(n-i, n)]_{1}, \epsilon \in\right] 0,1\right], x \in B_{(0,1)}^{n-i} \backslash\left(X_{\epsilon} \cap \bar{P}\right)\right\} .
\end{aligned}
$$

On a bien sûr : $G=\operatorname{adh}(E \cup F)$.

La projection naturelle $p: \bar{E} \longrightarrow[\bar{G}(n-i, n)]_{1} \times[0,1]$ est un morphisme sous-analytique propre. La fibre de $p$ au-dessus de $(\bar{P}, 0)$ est l'intersection $\mathcal{C}_{0} X \cap \bar{P}$ du cône tangent à l'origine de $X$ et de $\bar{P}$, autrement dit au-dessus de $\{\bar{P}\} \times[0,1], p$ est la déformation de $X \cap \bar{P}$ sur $\mathcal{C}_{0} X \cap \bar{P}$.

Le morphisme $p$ est stratifiable d'après [75], [29], [30], [35] ou [26] : il existe une stratification de Whitney sous-analytique $\Sigma$ de $G$ compatible avec $E$ et $F$, une stratification sousanalytique $\Sigma^{\prime}$ de $[\bar{G}(n-i, n)]_{1} \times[0,1]$, telles que la préimage par $p$ de toute strate $\sigma^{\prime}$ de $\Sigma^{\prime}$ soit réunion de strates de $\Sigma$, et la restriction de $p$ aux strates de $p^{-1}\left(\sigma^{\prime}\right)$ soit une submersion au-dessus de $\sigma^{\prime}$.

Dans ces conditions le premier lemme d'isotopie de Thom-Mather ([75], [54], [30]) assure que $p$ est topologiquement triviale au-dessus de chaque strate $\sigma^{\prime}$ de $\Sigma^{\prime}$, de façon compatible avec $\Sigma$. Comme $G$ est compact, le nombre de strates de $\Sigma^{\prime}$ est fini, et au-dessus de chacune d'elles, deux fibres quelconques $p^{-1}(\{(\bar{P}, \epsilon)\}), p^{-1}(\{(\bar{Q}, \eta)\})$ sont homéomorphes par un homéomorphisme respectant les fibres de $p$ dans $E$ et $F$. Or ces fibres dans $E$ sont précisément $X_{\epsilon} \cap \bar{P}$ et $X_{\eta} \cap \bar{Q}$; leur caractéristique d'Euler-Poincaré est ainsi la même.

- On en conclut que la famille d'entiers $\left(\chi\left(X_{\epsilon} \cap \bar{P}\right)\right)_{\left.\left.\bar{P} \in[\bar{G}(n-i, n)]_{1}, \epsilon \in\right] 0,1\right]}$ est une famille finie.

De plus, pour $\bar{P}$ fixé dans $[\bar{G}(n-i, n)]_{1}$, le segment $\{\bar{P}\} \times[0,1]$ rencontre chaque strate de $\Sigma^{\prime}$ un nombre fini de fois.

- On en conclut que la fonction $] 0,1] \ni \epsilon \longrightarrow \chi\left(X_{\epsilon} \cap \bar{P}\right) \in \mathbb{Z}$ converge. 
Pour terminer la preuve remarquons que par homogénéité de $\Lambda_{i}$, on a :

$$
\begin{aligned}
\frac{1}{\epsilon^{i}} \Lambda_{i}\left(X \cap B_{(0, \epsilon)}^{n}\right) & =\frac{1}{\epsilon^{i}} \int_{\bar{P} \in \bar{G}(n-i, n)} \chi\left(X \cap B_{(0, \epsilon)}^{n} \cap \bar{P}\right) \frac{d \bar{\gamma}_{n-i, n}(\bar{P})}{\beta(n, i)} \\
& =\int_{\bar{P} \in[\bar{G}(n-i, n)]_{1}} \chi\left(X_{\epsilon} \cap \bar{P}\right) \frac{d \bar{\gamma}_{n-i, n}(\bar{P})}{\beta(n, i)},
\end{aligned}
$$

or on vient de voir que la famille de fonctions $\left([\bar{G}(n-i, n)]_{1} \ni \bar{P} \longrightarrow \chi\left(X_{\epsilon} \cap \bar{P}\right)\right)_{\epsilon \in] 0,1]}$ est dominée par une fonction bornée et converge simplement. Le théorème de convergence dominée permet de conclure.

Le résultat de [44] devient ainsi un corollaire du théorème précédent (voir [50] pour la première mise en œuvre de la formule de Crofton dans la preuve de l'existence de la densité en tout point d'un ensemble semi-pfaffien).

Corollaire 1.4. - Soit $X$ un ensemble sous-analytique de dimension d dans $\mathbb{R}^{n}$. La densité d-dimensionnelle de $X$ en tout point de $\mathbb{R}^{n}$ existe.

Démonstration. - On applique le théorème 1.3 , avec $i=d$, en remarquant d'après la formule de Cauchy-Crofton pour le volume, que $\Lambda_{d}\left(X \cap B_{(0, \epsilon)}^{n}\right)=\mathcal{H}^{d}\left(X \cap B_{(0, \epsilon)}^{n}\right)$.

\section{Les invariants polaires $\sigma_{i}\left(X_{0}\right)$}

\subsection{Les invariants polaires réels}

Comme précédemment $X$ est un ensemble sous-analytique de $\mathbb{R}^{n}$ qui contient l'origine et qui représente le germe $X_{0}$ de dimension $d$. Si $P$ est un $i$-plan vectoriel de $\mathbb{R}^{n}$, nous identifierons au besoin $\pi_{P}(0)$ et 0 . Notons qu'il n'est pas sûr que la projection de $X_{0}$ sur un $i$-plan vectoriel $P$ de $\mathbb{R}^{n}$ soit bien définie ; c'est-à-dire que le germe en 0 du projeté de $X \cap B_{(0, r)}^{n}$ sur $P$ n'est peut-être pas indépendant de $r$. Il suffit de penser par exemple à l'éclatement $X$ de $\mathbb{R}^{2}$ de centre l'origine, projeté suivant la direction $P^{\perp}=\mathbb{P}^{1}(\mathbb{R}): \operatorname{si} B_{(x, r)}^{3}$ est une boule de $\mathbb{R}^{3}$ centrée en un point $x$ de l'axe $P^{\perp}$ de l'hélice $X$, la projection sur $P \operatorname{de} X \cap B_{(x, r)}^{3}$ n'est pas indépendante de $r$. Cependant cette situation n'est pas générique; on peut définir, pour des projections générales $\pi_{P}$ sur des $i$-plans vectoriels $P$ de $\mathbb{R}^{n}$, le projeté du germe $X_{0}$, c'est l'objet de la proposition 2.3.

Définition 2.1. - Soit $X$ un ensemble sous-analytique de $\mathbb{R}^{n}$ de dimension $d, i \in$ $\{0, \ldots n\}$ et $P \in G(i, n)$. On note $\mathcal{P}_{X}(P)$ la variété polaire de $X$ associée à $P$. Il s'agit de l'ensemble des points critiques de $\pi_{P \mid X^{\text {rég }}}: X^{\text {rég }} \rightarrow P$. C'est-à-dire que lorsque $i \leq d$ :

$$
\mathcal{P}_{X}(P)=\operatorname{adh}\left\{x \in X^{\text {rég }} ; \operatorname{dim}\left(\mathrm{T}_{x} X^{\text {rég }} \cap P^{\perp}\right) \geq d-i+1\right\},
$$

et lorsque $d<i, \mathcal{P}_{X}(P)=\operatorname{adh}(X)$. Nous noterons $\mathcal{D}_{X}(P)$ l'image de $\mathcal{P}_{X}(P)$ par la projection $\pi_{P}$ qui détermine $\mathcal{P}_{X}(P)$ et nous dirons que $\mathcal{D}_{X}(P)$ est l'image polaire de $X$ associée $\grave{a} P$. 
Nous rappelons maintenant le résultat qui stipule que les variétés polaires sont transverses aux directions auxquelles elles sont associées, ce qui nous permettra de définir les germes $\left(\pi_{P}\left(X \cap B_{(0, r)}^{n}\right)\right)_{0}$ et $\left(\mathcal{D}_{X \cap B_{(0, r)}^{n}}(P)\right)_{0}$ indépendamment de $r>0$ (Propositions 2.3 et 2.4).

Proposition 2.2 (Transversalité pour les variétés polaires absolues)

Avec les notations de la définition précédente, il existe un ouvert sous-analytique dense $\mathcal{F}_{X}^{i} \subset$ $G(i, n)$ tel que quel que soit $P \in \mathcal{F}_{X}^{i}$, il existe un voisinage ouvert $\mathcal{U}$ de 0 dans $\mathbb{R}^{n}$ tel que :

$$
\left(\mathcal{U} \cap \mathcal{P}_{X}(P) \cap P^{\perp}\right) \backslash\{0\}=\varnothing .
$$

Démonstration. - On procède par récurrence sur $i$. Lorsque $i \geq d$, on peut poser $: \mathcal{F}_{X}^{i}=$ $\left\{P \in G(i, n) ; P^{\perp} \cap C_{0} X=\{0\}\right\}$, qui est bien dense (cf. par exemple [11, Lemme 1.45]). Soit $i_{0} \leq d$ et supposons montrée l'existence de $\mathcal{F}_{X}^{j}$ pour $i_{0} \leq j \leq d$. Soit $P \in \mathcal{F}_{X}^{i_{0}}$. Par hypothèse, il existe un voisinage ouvert $\mathcal{U}$ de 0 dans $\mathbb{R}^{n}$ tel que : $\left(\mathcal{U} \cap \mathcal{P}_{X}(P) \cap P^{\perp}\right) \backslash\{0\}=\varnothing$. Soit $\ell$ une droite (générale) de $P$ ne coupant pas $\mathcal{D}_{X}(P) \backslash\{0\}$, dans un voisinage $\mathcal{U}^{\prime}$ de 0 dans $P$. On en déduit que $\mathcal{U} \cap \pi_{P}^{-1}\left(\mathcal{U}^{\prime}\right) \cap\left(\ell \oplus P^{\perp}\right)$ ne coupe pas $\mathcal{P}_{X}(P) \backslash\{0\}$. En notant $P^{\prime}=\left(\ell \oplus P^{\perp}\right)^{\perp}$ et en remarquant que $\mathcal{P}_{X}\left(P^{\prime}\right) \subset \mathcal{P}_{X}(P)$, on obtient :

$$
\left(\mathcal{U} \cap \pi_{P}^{-1}\left(\mathcal{U}^{\prime}\right) \cap \mathcal{P}_{X}\left(P^{\prime}\right) \cap P^{\prime \perp}\right) \backslash\{0\}=\varnothing,
$$

ce qui termine la preuve, puisque $\operatorname{dim}\left(P^{\prime}\right)=i_{0}-1$.

On peut maintenant prouver que l'image d'un germe est encore un germe pour des projections génériques.

Soit $X$ un ensemble sous-analytique borné de $\mathbb{R}^{n}$ dont l'adhérence contient l'origine et soient $i \in\{0, \ldots, n\},\left(X^{j}\right)_{j \in\{0, \cdots, k\}}$ une stratification sous-analytique finie de $\operatorname{adh}(X)$, sans condition de régularité particulière. Rappelons que d'après la proposition 2.2 et avec les notations de celle-ci, quel que soit $P$ dans l'ouvert sous-analytique dense $\bigcap_{j=0}^{k} \mathcal{F}_{X^{j}}^{i}$ de $G(i, n)$, quel que soit $j \in\{0, \cdots, k\}$, il existe $r_{P}>0$ tel que : $\left(B_{\left(0, r_{P}\right)} \cap \mathcal{P}_{X^{j}}(P) \cap P^{\perp}\right) \backslash\{0\}=\varnothing$.

Notation. - Dans la suite on notera $\mathcal{E}_{X}^{i}$ pour $\bigcap_{j=0}^{k} \mathcal{F}_{X^{j}}^{i}$. Cet ensemble est associé à une stratification de $\operatorname{adh}(X)$, que l'on pourra choisir selon la situation. On précisera, quand cela sera nécessaire, la stratification à laquelle $\mathcal{E}_{X}^{i}$ est attaché.

Proposition 2.3 (Projections sans éclatement). - Soient $X$ un ensemble sous-analytique borné de $\mathbb{R}^{n}$ dont l'adhérence contient l'origine et $i \in\{0, \ldots, n\}$. Avec les notations qui précèdent, quel que soit $P$ dans l'ouvert sous-analytique dense $\mathcal{E}_{X}^{i}$ de $G(i, n)$, pour tout $\left.\left.r \in\right] 0, r_{P}\right]$, il existe $s>0$ vérifiant :

$$
B_{(0, s)} \cap \pi_{P}\left(X \cap B_{(0, r)}\right)=B_{(0, s)} \cap \pi_{P}\left(X \cap B_{\left(0, r_{P}\right)}\right) .
$$

Autrement dit, une projection générique de $X_{0}$ sur un i-plan de $G(i, n)$ définit bien un germe dans $P_{0}$.

REMARQue. - La preuve qui suit montre en réalité que dès que $\mathcal{U}$ est un voisinage de 0 dans $\mathbb{R}^{n}$ tel que pour tout $j \in\{0, \cdots, k\},\left(\mathcal{U} \cap P^{\perp} \cap \mathcal{P}_{X_{j}}(P)\right) \backslash\{0\}=\varnothing$, le germe $\pi_{P}(\mathcal{U} \cap X)$ est bien défini. 
Démonstration. - Soient $i \in\{0, \ldots, n\}$ et $P \in G(i, n)$. S'il existe $r \in] 0, r_{P}[$ tel que quel que soit $s>0, B_{(0, s)} \cap \pi_{P}\left(X \cap B_{\left(0, r_{P}\right)}\right) \neq B_{(0, s)} \cap \pi_{P}\left(X \cap B_{(0, r)}\right)$, on obtient une suite de points $\left(x_{\ell}^{r}\right)_{\ell \in \mathbb{N}}$ dans $X$ telle que, si $y_{\ell}^{r}=\pi_{p}\left(x_{\ell}^{r}\right), r<\left\|x_{\ell}^{r}\right\|<r_{P}, \lim _{\ell \rightarrow \infty} y_{\ell}^{r}=0$, et $\pi_{P}^{-1}\left(\left\{y_{\ell}^{r}\right\}\right) \cap B_{(0, r)}^{n}=\varnothing$. Quitte à extraire une sous-suite de $\left(x_{\ell}^{r}\right)_{\ell \in \mathbb{N}}$, on peut supposer que $\left(x_{\ell}^{r}\right)_{\ell \in \mathbb{N}}$ converge vers $x_{r} \in\left(P^{\perp} \cap \operatorname{adh}(X) \cap B_{\left(0, r_{P}\right)}^{n}\right) \backslash \operatorname{int}\left(B_{(0, r)}\right)$. Il existe alors un indice $j \in\{0, \ldots, k\}$ pour lequel $X^{j}$ contient $x_{r}$. Maintenant, si $P$ est dans $\mathcal{E}_{X^{j}}^{i}$, pour tout $x \in X^{j} \cap P^{\perp} \cap B_{\left(0, r_{P}\right)}^{n}, \mathrm{~T}_{x} X^{j}$ est transverse à $P^{\perp}$. Dans ce cas :

- Soit $i>\operatorname{dim}\left(X^{j}\right)$, et $P^{\perp}$ et $X^{j}$ ne se coupent pas dans $B_{\left(0, r_{P}\right)}$, ce qui contredit l'existence de $x_{r}$.

- Soit $i \leq \operatorname{dim}\left(X^{j}\right)$, et tout un voisinage de $x_{r}$ dans $X^{j}$ se projette sur tout un voisinage de 0 dans $P$. Si $X$ est fermé, $X^{j} \subset \operatorname{adh}(X)=X$, donc tout un voisinage de $x$ dans $X$ se projette sur tout un voisinage de 0 dans $P$, ce qui contredit l'existence de la suite $\left(x_{\ell}^{r}\right)_{\ell \in \mathbb{N}}$.

Si $X$ n'est pas fermé, après avoir prouvé que le projeté du germe de $F^{0}=\operatorname{adh}(X)$ définit bien un germe, pour une projection générique, on applique le même raisonnement à $F^{1}=$ $\operatorname{adh}(\operatorname{adh}(X) \backslash X)$, puis à $F^{2}=\operatorname{adh}\left(F^{1} \backslash(\operatorname{adh}(X) \backslash X)\right)$ etc. Les projetés génériques de chacun des germes de ces fermés définissent bien des germes. Il en est alors de même du germe de $X$.

Proposition 2.4. - Soient $X$ un ensemble sous-analytique de $\mathbb{R}^{n}$ contenant l'origine, $i \in$ $\{0, \ldots, n\}$ et $\mathcal{E}_{X}^{i} \subset G(i, n)$ l'ouvert sous-analytique dense introduit ci-dessus et relatif à une stratification $\left(X^{j}\right)_{j \in\{0, \cdots, k\}}$ de $\operatorname{adh}(X)$. Quels que soient $\left.\left.P \in \mathcal{E}_{X}^{i}, r \in\right] 0, r_{P}\right]$, il existe $s>0$ vérifiant :

$$
\forall j \in\{0, \cdots, k\}, \quad B_{(0, s)}^{i} \cap \mathcal{D}_{X^{j} \cap B_{(0, r)}^{n}}(P)=B_{(0, s)}^{i} \cap \mathcal{D}_{X^{j} \cap B_{\left(0, r_{P}\right)}^{n}}(P),
$$

autrement dit pour des projections générales de $G(i, n)$, les images polaires du germe $X_{0}$ sont bien définies.

Démonstration. - D'après la proposition 2.3, la condition $\left(B_{\left(0, r_{P}\right)} \cap \mathcal{P}_{X^{j}}(P) \cap P^{\perp}\right) \backslash$ $\{0\}=\varnothing$ assure la conclusion de la proposition 2.4.

Proposition 2.5. - Soient $X$ un ensemble sous-analytique fermé de $\mathbb{R}^{n}$ contenant l'origine, $i \in\{0, \ldots, n\}$ et $P \in \mathcal{E}_{X}^{i}$, où $\mathcal{E}_{X}^{i}$ est relatif à une stratification (a)-régulière $\left(X^{j}\right)_{j \in\{0, \cdots, k\}}$ de $X$. Il existe alors $r_{P}^{\prime}>0$, tel que quel que soit $\left.\left.r \in\right] 0, r_{P}^{\prime}\right]$, quel que soit $j \in\{0, \cdots, k\}$ :

$$
0 \notin \mathcal{D}_{L_{X^{j}}^{r}}(P)
$$

où $L_{X^{j}}^{r}=S_{(0, r)}^{n-1} \cap X^{j}$ est le link de $X^{j}$ dans $S_{(0, r)}^{n-1}$.

Démonstration. - Soit $\rho>0$ tel que $S_{\left(0, \rho^{\prime}\right)}$ soit transverse aux strates de $\left(X^{j}\right)_{j \in\{0, \cdots, k\}}$, dès que $0<\rho^{\prime}<\rho$. Avec les notations précédentes, soit $\left.r \in\right] 0, \min \left(\rho, r_{P}\right)[$. Commençons par remarquer que quelle que soit la strate $X^{j}$, celle-ci est transverse à $\pi_{P}^{-1}\left(\pi_{P}(x)\right)=$ $\pi_{P}(x)+P^{\perp}$ en $x$, pour $x$ dans $S(0, r)$, pourvu que $\pi_{P}(x)$ soit suffisamment proche de 0 . Ceci résulte de la $(a)$-régularité de la stratification $\left(X^{j}\right)_{j \in\{0, \cdots, k\}}$, du fait que $X$ est fermé et du fait que $P^{\perp}$ ne coupe pas $\mathcal{P}_{X^{j}}(P)$ dans $S(0, r)$. Raisonnons par l'absurde pour prouver la proposition 2.5: si $0 \in \operatorname{adh}\left(\mathcal{D}_{L^{r} X^{j}}(P)\right)$, il existe une suite $\left(x_{\ell}^{r}\right)_{\ell \in \mathbb{N}}$ de $S_{(0, r)} \cap X^{j}$ telle que 
$\mathrm{T}_{x_{\ell}^{r}} L_{X^{j}}^{r}=\mathrm{T}_{x_{\ell}^{r}} S_{(0, r)} \cap \mathrm{T}_{x_{\ell}^{r}} X^{j}$ et $P^{\perp}$ soient non transverses et $\lim _{\ell \rightarrow \infty} \pi_{P}\left(x_{\ell}^{r}\right)=0$. Comme $\pi_{P}\left(x_{\ell}^{r}\right)+P^{\perp}$ est transverse en $x_{\ell}^{r}$ à $X^{j}$, on en déduit que : $\mathrm{T}_{x_{\ell}^{r}} X^{j} \cap P^{\perp} \subset \mathrm{T}_{x_{\ell}^{r}} S_{(0, r)}$. Une sous-suite de $\left(x_{\ell}^{r}\right)_{\ell \in \mathbb{N}}$ converge vers un point $x^{r}$ de $S_{(0, r)} \cap X$, qui est dans une strate $X^{m}$ et à nouveau par la condition (a), on a : $\mathrm{T}_{x^{r}} X^{m} \cap P^{\perp} \subset \mathrm{T}_{x^{r}} S_{\left(0,\left\|x^{r}\right\|\right)}$. En faisant maintenant varier $r$, on en déduit une suite non constante $\left(x_{p}\right)_{p \in \mathbb{N}}$ contenue dans une strate $X^{m}$, de limite 0 et telle que :

$$
\mathrm{T}_{x_{p}} X^{m} \cap P^{\perp} \subset \mathrm{T}_{x_{p}} S_{\left(0,\left\|x_{p}\right\|\right)} .
$$

Mais comme $P^{\perp}$ et $X^{m}$ sont transverses dans $B_{\left(0, r_{P}\right)}$, on a :

$$
\mathrm{T}_{x_{p}}\left(X^{m} \cap P^{\perp}\right)=\mathrm{T}_{x_{p}} X^{m} \cap P^{\perp} \subset \mathrm{T}_{x_{p}} S_{\left(0,\left\|x_{p}\right\|\right)} .
$$

Or $X^{m} \cap P^{\perp}$ est un sous-analytique de dimension $\neq 0$ dont l'adhérence contient 0 ; l'inclusion $\mathrm{T}_{x_{p}}\left(X^{m} \cap P^{\perp}\right) \subset \mathrm{T}_{x_{p}} S_{\left(0,\left\|x_{p}\right\|\right)}$ est donc en contradiction avec le lemme de Whitney.

REMARQUe. - La preuve montre que l'on peut prendre $r_{P}^{\prime}$ de sorte qu'en tout point $x$ de $B\left(0, r_{P}^{\prime}\right), \mathrm{T}_{x}\left(X^{m} \cap P^{\perp}\right)$ et la direction $x$ ne sont pas orthogonaux, quelle que soit la strate $X^{m}$.

Nous rassemblons maintenant en un théorème les diverses propositions établies, et nous introduisons comme dans [11] le discriminant local $\Delta_{X_{0}}(P)$, les profils polaires locaux $\mathcal{K}_{\ell}^{P}$, et les caractéristiques $\chi_{\ell}^{P}$ du germe $X_{0}$, associés à une projection générale $\pi_{P}$, pour $P \in$ $G(i, n)$ et $i \in\{0, \ldots, n\}$. L'introduction de ces objets et la formule de représentation intégrale pour les courbures de Lipschitz-Killing locales $\Lambda_{i}^{\text {loc }}\left(X_{0}\right)$ que nous allons démontrer (Théorème 3.1) nous permet de ramener l'étude du comportement des courbures $\Lambda_{i}^{\text {loc }}\left(X_{0}\right)$ à la géométrie des discriminants $\Delta_{X_{0}}\left(P^{i}\right), \ldots, \Delta_{X_{0}}\left(P^{d}\right)$, (avec $P^{i}, \ldots, P^{d}$ généraux dans respectivement $G(i, n), \ldots, G(d, n)$ ) qui est bien comprise le long de strates de Verdier (cf. Théorème 4.10).

THÉORÈme 2.6. - Soit $X$ un ensemble sous-analytique fermé et borné de $\mathbb{R}^{n}$ de dimension d contenant l'origine et $\left(X^{j}\right)_{j \in\{0, \ldots, k\}}$ une stratification de Whitney de $X$. Soit $i \in\{0, \ldots, n\}$.

Avec les notations qui précèdent la proposition 2.3, quel que soit $P$ dans l'ouvert sousanalytique dense $\mathcal{E}_{X}^{i}=\bigcap_{j=1}^{k} \mathcal{F}_{X^{j}}^{i}$ de $G(i, n)$ :

(i) Quel que soit $r>0$ suffisamment petit, quel que soit $j \in\{0, \ldots, k\}$, le germe $\left[\mathcal{D}_{X^{j} \cap B_{(0, r)}^{n}}(P)\right]_{0}$ est indépendant de $r$, on note $\Delta_{X_{0}}(P)$ le germe $\left[\bigcup_{j=0}^{k} \mathcal{D}_{X^{j} \cap B_{(0, r)}^{n}}(P)\right]_{0}$ et on l'appelle le discriminant local de $X_{0}$ associé à $P$.

(ii) Le germe $\left[\pi_{P}\left(X \cap B_{(0, r)}^{n}\right)\right]_{0} \backslash \Delta_{X_{0}}(P)$ est bien défini pour $r$ suffisamment petit. Il s'agit du germe d'un ouvert sous-analytique de $P$. Les germes de ses composantes connexes sont notés $\mathcal{K}_{1}^{P}, \ldots, \mathcal{K}_{n_{P}}^{P}$ et sont appelés les profils polaires locaux (à l'origine) de $X_{0}$ associés $\grave{a} P$.

(iii) Soit $\rho>0$ suffisamment petit pour que $S_{\left(0, \rho^{\prime}\right)}^{n-1}$ soit transverse à chaque $X^{j}$, lorsque $\rho^{\prime} \in$ ] $0, \rho[$. Notons alors, pour $r \in] 0, \rho\left[\right.$ et pour $j \in\{0, \ldots, k\}, L_{X^{j}}^{r}=X^{j} \cap S_{(0, r)}^{n-1}$, puis $\mathcal{D}_{L^{r}}(P)=\bigcup_{j=0}^{k} \pi_{P}\left(\mathcal{P}_{L_{X j}^{r}}(P)\right)$ le discriminant du link $L^{r}=X \cap S_{(0, r)}^{n-1}$.

Le complémentaire de $\mathcal{D}_{L^{r}}(P) \cup \bigcup_{j=0}^{k} \mathcal{D}_{X^{j} \cap B_{(0, r)}^{n}}(P)$ dans $\pi_{P}\left(X \cap B_{(0, r)}^{n}\right)$ est un ouvert sous-analytique dense. On note chacune de ses composantes connexes $\mathrm{K}_{1}^{P, r}, \ldots, \mathrm{K}_{N_{P}}^{P, r}$. Pour $r$ suffisamment petit, les germes des ouverts $\mathrm{K}_{j}^{P, r}$ qui adhèrent 
à 0 sont les profils polaires locaux $\mathcal{K}_{1}^{P}, \ldots, \mathcal{K}_{n_{P}}^{P}$ de $X_{0}$. De plus à chaque $\mathcal{K}_{j}^{P}$ on peut associer un entier $\chi_{j}^{P} \in \mathbb{Z}$ égal à $\chi\left(\pi_{P}^{-1}(y) \cap X \cap B_{(0, r)}^{n}\right)$, cet entier ne dépendant ni de $r>0$, qui est supposé suffisamment petit, ni de $y \in \mathrm{K}_{j}^{P, r}$, pourvu que $\|y\| \ll r$.

Démonstration. - (i) résulte de la proposition 2.4 et (ii) résulte de la proposition 2.3.

Prouvons (iii) : par la proposition 2.5 les images polaires des $L_{X^{j}}^{r}$ associées à $P$ n'adhèrent pas à l'origine, pour $r$ petit, de sorte que les $\mathrm{K}_{j}^{P, r}$ qui adhèrent à l'origine n'ont pas dans leur bord, au voisinage de l'origine, de points communs avec les images polaires des $L_{X^{j}}^{r}$; au voisinage de l'origine le bord de $\mathrm{K}_{j}^{P, r}$ est obtenu comme la réunion des images polaires des $X_{j}$. Les germes de tels $\mathrm{K}_{j}^{P, r}$ donnent donc les profils polaires locaux $\mathcal{K}_{1}^{P}, \ldots, \mathcal{K}_{n_{P}}^{P}$ de $X_{0}$.

Enfin montrons que les entiers relatifs $\chi_{j}^{P, r}=\chi\left(\pi_{P}^{-1}(y) \cap X \cap B_{(0, r)}^{n}\right)$ ne dépendent ni de $y \in \mathrm{K}_{j}^{P, r}$, ni de $r$, pourvu que ce dernier soit choisi petit, et que la partition $\left(X^{0}, \ldots, X^{k}\right)$ soit une stratification de Whitney. On commence par remarquer que, pour $r$ fixé, quels que soient $y$ et $z$ dans $\mathrm{K}_{j}^{P, r}$, les fibres $\pi_{P}^{-1}(y) \cap X \cap B_{(0, r)}^{n}$ et $\pi_{P}^{-1}(z) \cap X \cap B_{(0, r)}^{n}$ sont homéomorphes. Il s'agit une fois de plus du premier lemme d'isotopie de Thom-Mather; le morphisme sous-analytique propre $\pi_{P}: X \cap B_{(0, r)}^{n} \rightarrow P \cap B_{(0, r)}^{n}$ étant stratifié par $\left(X^{j}\right)_{j \in\{0, \ldots, k\}}$ et $\left(\mathrm{K}_{j}^{P, r}\right)_{j \in\left\{1, \ldots, N_{P}\right\}}$.

On montre ensuite qu'à $P$ fixé dans $\mathcal{E}_{X}^{i}$, pour $r$ suffisamment proche de 0 , l'entier $\chi_{j}^{P, r}$ associé à $\mathrm{K}_{j}^{P, r}$, où $j \in\left\{1, \ldots, n_{P}\right\}\left(\mathrm{K}_{j}^{P, r}\right.$ adhère à l'origine), est indépendant de $r$. Considérons pour cela $E=\left\{(y, r) ; y \in \operatorname{adh}\left(\mathrm{K}_{j}^{P, r}\right), r \in[0, \epsilon]\right\}(\epsilon>0$ suffisamment petit), $F=$ $\left\{(x, r) ; \pi_{P}(x) \in \operatorname{adh}\left(\mathrm{K}_{j}^{P, r}\right), x \in X \cap B_{(0, r)}^{n}, r \in[0, \epsilon]\right\}$, et $p: F \rightarrow E$ définie par $p(x, r)=$ $\left(\pi_{P}(x), r\right)$. Il existe une stratification $\Sigma^{\prime}$ de $F$, une stratification finie $\Sigma$ de $E$, compatible avec $E^{\prime}=\left\{(y, r) ; y \in \mathrm{K}_{j}^{P, r}, r \in[0, \epsilon]\right\}$, qui stratifient $p$. Soit $\sigma$ une strate de $\Sigma$ qui soit contenue dans $E^{\prime}$ et qui adhère au voisinage de l'origine à tout un segment $\left[0, \epsilon^{\prime}\right] \subset[0, \epsilon]$. Si $r, r^{\prime} \leq \epsilon^{\prime}$, et si $(y, r)$ et $\left(z, r^{\prime}\right)$ sont suffisamment proches de $(0, r)$ et $\left(0, r^{\prime}\right)$ respectivement dans $\left(\mathrm{K}_{j}^{P, r} \times\{r\}\right) \cap \sigma$ et $\left(\mathrm{K}_{j}^{P, r^{\prime}} \times\{r\}\right) \cap \sigma$, les fibres $p^{-1}(y, r)$ et $p^{-1}\left(z, r^{\prime}\right)$ étant homéomorphes et de caractéristique d'Euler-Poincaré respectivement $\chi_{j}^{P, r}$ et $\chi_{j}^{P, r^{\prime}}$, on en déduit l'indépendance de $\chi_{j}^{P, r}$ relativement à $r<\epsilon^{\prime}$.

Remarque. - Dans le théorème 2.6.(iii), on peut choisir $r<r_{P}^{\prime}\left(r_{P}^{\prime}\right.$ donné par la proposition 2.5) et $y$ dans $P$ de sorte que $\|y\|<\delta$, où $\delta>0$ minore la distance de $\mathcal{D}_{L^{r}}(P)$ à 0 .

L'introduction des profils polaires locaux $\left(\mathcal{K}_{j}^{P}\right)_{j \in\left\{1, \ldots, n_{P}\right\}, P \in \mathcal{E}_{X}^{i}}$ et des caractéristiques $\left(\chi_{j}^{P}\right)_{j \in\left\{1, \ldots, n_{P}\right\}, P \in \mathcal{E}_{X}^{i}}$, qui leur sont affectées nous permet de définir des invariants polaires : les moyennes sur $G(i, n)$ des densités des profils polaires locaux de $X_{0}$ associés à $P$, affectées des caractéristiques locales $\chi_{j}^{P}$.

Définition 2.7. - Avec les notations du théorème 2.6, nous définissons, pour $i \in$ $\{0, \ldots, n\}$, des invariants polaires $\sigma_{i}\left(X_{0}\right)$ par :

$$
\sigma_{i}\left(X_{0}\right)=\int_{P \in \mathcal{E}_{X}^{i}} \sum_{j=1}^{n_{P}} \chi_{j}^{P} \cdot \Theta_{i}\left(\mathcal{K}_{j}^{P}\right) d \gamma_{i, n}(P) .
$$

On note $\sigma_{*}\left(X_{0}\right)$ la suite $\left(\sigma_{i}\left(X_{0}\right)\right)_{i \in\{0, \cdots, n\}}$. 
Notons maintenant $\mathcal{C}(X)$ le groupe des fonctions constructibles sur $X$, c'est-à-dire les fonctions du type : $\varphi=\sum_{j=1}^{N} n_{j} \cdot 1_{K^{j}}$, pour $n_{j} \in \mathbb{Z}, K^{j}$ des sous-analytiques de $X$, et pour $Z \subset X$ et $y \in Y, f_{*}\left(1_{Z}\right)(y)=\chi\left(f^{-1}(y) \cap Z\right)$. Si on considère le foncteur suivant de la catégorie des ensembles sous-analytiques compacts à la catégorie des groupes :

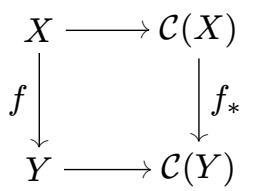

en vertu du théorème 2.6 , pour $\pi_{P}$ une projection générale dans $G(i, n)$, ce diagramme admet l'équivalent local :

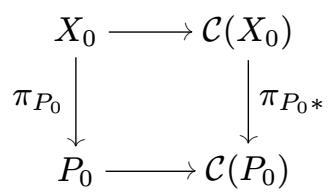

où pour $Z_{0} \subset X_{0}$ et $y \in P, \pi_{P_{0} *}\left(1_{Z_{0}}\right)(y)=\chi\left(\pi_{P}^{-1}(y) \cap Z \cap B(0, r)\right), r$ étant suffisamment petit, et $0<\|y\| \ll r$. Si l'on note ensuite $\operatorname{par} \theta(\varphi)$ l'intégrale relativement à la densité locale en 0 d'un germe $\varphi: P_{0} \rightarrow \mathbb{Z}$ de fonction constructible, c'est-à-dire : $\theta(\varphi)=\sum_{j=1}^{N} n_{j} \cdot \Theta\left(K_{0}^{j}\right)$ lorsque $\varphi=\sum_{j=1}^{N} n_{j} \cdot 1_{K_{0}^{j}}$, pour des germes d'ensembles sous-analytiques $K_{0}^{j} \subset P_{0}$, on obtient la formule : $\sigma_{i}\left(X_{0}\right)=\int_{P \in G(i, n)} \theta\left(\pi_{P_{0} *}\left(1_{X_{0}}\right)\right) d P$.

La figure de la page suivante compare, pour $X_{0}$ un germe de surface de $\mathbb{R}^{3}$ et pour une projection $\pi_{P}$ particulière sur un 2-plan de $\mathbb{R}^{3}$, ce qui est respectivement pris en compte dans le calcul de $\sigma_{2}\left(X_{0}\right)$ et $\Lambda_{2}^{\text {loc }}\left(X_{0}\right)$.

Sur cet exemple, on a :

- $\theta\left(\pi_{P_{0} *}\left(1_{X_{0}}\right)\right)=\chi_{1}^{P} \cdot \Theta_{2}\left(\left(K_{1}^{P}\right)_{0}\right)+\chi_{2}^{P} \cdot \Theta_{2}\left(\left(K_{2}^{P}\right)_{0}\right)+\chi_{4}^{P} \cdot \Theta_{2}\left(\left(K_{4}^{P}\right)_{0}\right)$ et $\sigma_{2}\left(X_{0}\right)=$ $\int_{Q \in G(2,3)} \theta\left(\pi_{Q_{0} *}\left(1_{X_{0}}\right)\right) d Q$. Notons que dans le calcul de $\theta\left(\pi_{P_{0} *}\left(1_{X_{0}}\right)\right)$, pour la projection sur $P$, le domaine $K_{3}^{P}$ (en gris sur le dessin) n'est pas pris en compte, puisque seuls les profils polaires locaux adhérents à l'origine comptent dans la définition de $\sigma_{i}$. Ces profils sont ceux déterminés par $\Delta_{X_{0}}(P)$.

- En revanche dans le calcul de $\Lambda_{2}^{\text {loc }}\left(X_{0}\right)$ le terme $\chi_{3}^{P} \cdot \mathcal{H}^{2}\left(K_{3}^{P}\right)$ est pris en compte, car tous les domaines $K_{j}^{P, r}$ interviennent dans ce calcul, y compris ceux qui ne contiennent pas 0 dans leur adhérence. En notant $v\left(\pi_{P *}\left(1_{X \cap B_{0, r}}\right)\right)=\sum_{j=1}^{4} \chi_{j}^{P} \cdot \mathcal{H}^{2}\left(K_{0}^{j}\right)$, on a : $\Lambda_{2}^{\operatorname{loc}}\left(X_{0}\right)=\lim _{r \rightarrow 0} \frac{1}{\alpha_{2} r^{2}} \int_{Q \in G(2,3)} v\left(\pi_{Q *}\left(1_{X \cap B_{(0, r)}^{n}}\right)\right) d Q$.

Remarques. - Trivialement, lorsque $i=0, \sigma_{i}\left(X_{0}\right)=1=\Lambda_{0}^{\text {loc }}\left(X_{0}\right)$ et lorsque $i=n$, $\sigma_{i}\left(X_{0}\right)=\Theta_{n}\left(X_{0}\right)=\Lambda_{n}^{\text {loc }}\left(X_{0}\right)$. Enfin lorsque $i=d=\operatorname{dim}\left(X_{0}\right), \sigma_{i}\left(X_{0}\right)=\Theta_{d}\left(X_{0}\right)$, c'està-dire $\sigma_{i}\left(X_{0}\right)=\Lambda_{d}^{\text {loc }}\left(X_{0}\right)$. Cette dernière égalité, contrairement aux deux autres n'est pas immédiate (cf. [11]) :

ThÉORÈme 2.8 (Formule de Cauchy-Crofton locale, [11, Théorèmes 1.10 et 1.16])

Soit $X$ un sous-ensemble sous-analytique de $\mathbb{R}^{n}$ de dimension d et soient $\mathcal{G} \subset G(d, n)$ un sous-ensemble sous-analytique de $G(d, n)$ sur lequel agit transitivement un sous-groupe $G$ de $\mathcal{O}_{n}(\mathbb{R})$ et $\mu_{d, n}$ une mesure $G$-invariante sur $\mathcal{G}$, tels que : 


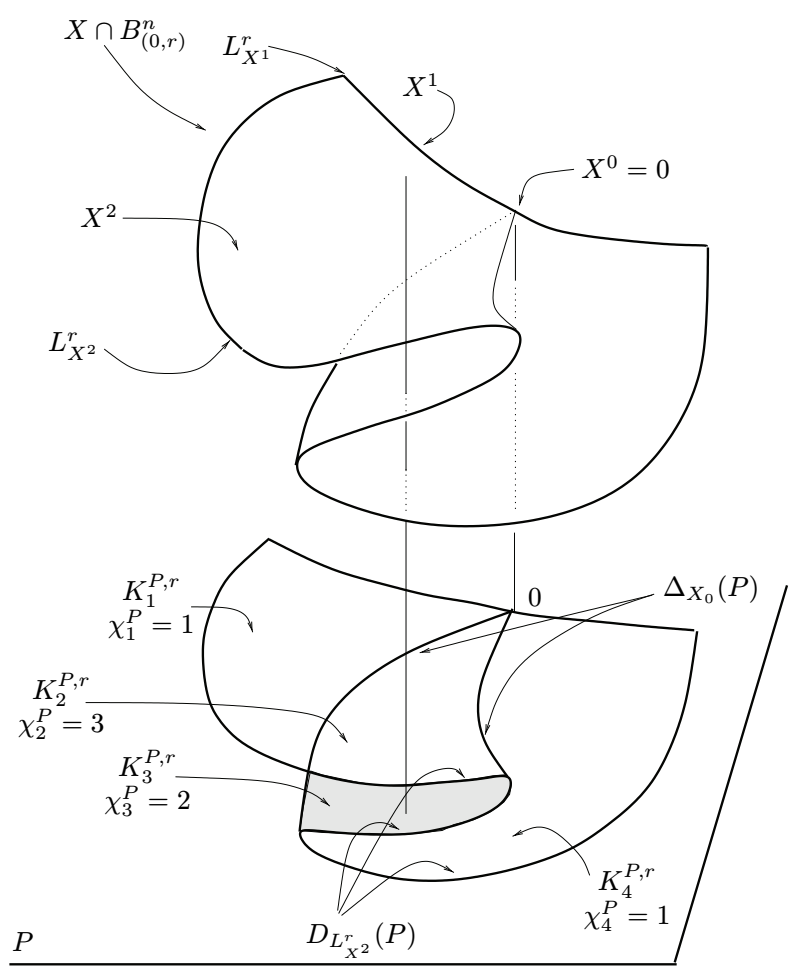

- les espaces tangents à $\mathcal{C}_{0} X_{0}$ soient dans $\mathcal{G}$;

- il existe $P^{0} \in \mathcal{G}$ dont le fixateur $G_{P^{0}}$ agit transitivement sur le d-espace vectoriel sousjacent à $P^{0}$ et $\mu_{d, n}(\mathcal{G})=\mu_{d, n}\left(\mathcal{G} \cap \mathcal{E}_{X}^{d}\right)=1$.

L'égalité suivante a alors lieu:

$$
\int_{P \in \mathcal{G}_{\mathcal{E}} \mathcal{E}_{X}^{d}} \sum_{j=1}^{n_{P}} \chi_{j}^{P} \cdot \Theta_{d}\left(\mathcal{K}_{j}^{P}\right) d \mu_{d, n}(P)=\Theta_{d}\left(X_{0}\right) .
$$

Dans le cas où $\mathcal{G}=G(d, n)$ et $G=\mathcal{O}_{n}(\mathbb{R})$, la formule donne $: \sigma_{d}\left(X_{0}\right)=\Lambda_{d}^{\text {loc }}\left(X_{0}\right)$.

Dans le cas où $X$ est analytique complexe, $\mathcal{G}=\widetilde{G}(d / 2, n)$ et $G=U_{n}(\mathbb{C})$, la formule donne: $\Theta_{d}\left(X_{0}\right)=e(X, 0)$.

Remarque 2.9. - Lorsque $\left(X^{j}\right)_{j \in\{0, \cdots, k\}}$ est une stratification de Whitney de $X, 0 \in$ $X^{0}, \sigma_{i}\left(X_{0}\right)=1$, lorsque $i \leq \operatorname{dim}\left(X^{0}\right)$. En effet, soit une direction de projection générale $P^{\perp}\left(P \in \mathcal{E}_{X}^{i}\right)$, transverse à $X^{0}$ en 0 . Comme la stratification $\left(X^{j}\right)_{j \in\{0, \cdots, k\}}$ est $(a)$-régulière, $\pi_{P}$ est transverse aux strates adjacentes à $X^{0}$ en 0 , et d'après la proposition $2.5, \pi_{P}$ est aussi transverse aux strates du link $X \cap S_{(0, r)}^{n}$ induites par $\left(X^{j}\right)_{j \in\{0, \cdots, k\}}$, ceci au-dessus d'un voisinage $P \cap B_{(0, \eta)}^{n}$ de 0 dans $P$, pour $0<\eta \ll r$. Il en résulte que la restriction $\pi_{r}$ de $\pi_{P}$ à $X \cap B_{(0, r)}^{n}$, pour $r$ suffisamment petit est une submersion propre au-dessus de $P \cap B_{(0, \eta)}^{n}$, stratifiée par $\left(X^{j} \cap \operatorname{int}\left(B_{(0, r)}^{n}\right), X^{j} \cap S_{(0, r)}^{n}\right)_{j \in\{0, \cdots, k\}}$ et les projections de ces strates dans $P \cap B_{(0, \eta)}^{n}$. Les fibres de $\pi_{r}$ au-dessus de $P \cap B_{(0, \eta)}^{n}$ sont toutes homéomorphes et puisque 
$\pi_{r}^{-1}(\{0\})$ est contratile, on a bien : $\sigma_{i}\left(X_{0}\right)=1$. En conclusion, si $\left(X^{j}\right)_{j \in\{0, \cdots, k\}}$ est une stratification de Whitney de $X$ et si $d_{0}$ est la dimension de la strate qui contient 0 , on a :

$$
\sigma_{*}\left(X_{0}\right)=\left(1, \cdots, 1, \sigma_{d_{0}+1}\left(X_{0}\right), \cdots, \sigma_{d-1}\left(X_{0}\right), \Lambda_{d}^{\text {loc }}\left(X_{0}\right), 0, \cdots, 0\right) .
$$

Remarque. - Au même titre que les invariants $\Lambda_{i}$, et donc au même titre que les courbures locales $\Lambda_{i}^{\text {loc }}$, les invariants polaires $\sigma_{i}$ sont intrinsèques : si le germe $X_{0} \subset \mathbb{R}_{0}^{n} \subset \mathbb{R}_{0}^{n+1}$, $\sigma_{i, n}\left(X_{0}\right)=\sigma_{i, n+1}\left(X_{0}\right)$, où $\sigma_{i, n}\left(X_{0}\right)$ désigne l'invariant $\sigma_{i}\left(X_{0}\right)$, calculé grâce à $G(i, n)$. Il s'agit à nouveau d'une conséquence de la formule de Cauchy-Crofton pour la densité. Comme c'est le cas pour les invariants $\Lambda_{i}^{\text {loc }}$, il est facile de s'assurer que les invariants $\sigma_{i}$ ne dépendent pas de la dimension de l'espace euclidien dans lequel $X_{0}$ est considéré. De plus, pour $i \in\{0, d, d+1, \cdots, n\}$, les invariants polaires $\sigma_{i}$ sont égaux aux courbures locales $\Lambda_{i}^{\text {loc }}$. Nous allons établir dans la section suivante, au théorème 3.1, que les courbures $\Lambda_{i}^{\text {loc }}$ s'obtiennent en réalité comme des combinaisons linéaires (à coefficients universels) des invariants $\sigma_{j}$; les égalités et les propriétés communes que nous venons d'observer ne sont que des cas spéciaux de ce théorème.

\subsection{Les invariants polaires complexes}

On peut bien sûr définir les invariants polaires $\sigma_{i}\left(X_{0}\right)$ dans le cadre complexe, lorsque $X_{0}$ est un germe d'ensemble analytique complexe à l'origine de $\mathbb{C}^{n}$; pour cela il suffit de ne considérer, dans la définition 2.7, que les $i$-plans complexes de $\mathbb{C}^{n}$ appartenant à l'équivalent complexe $\widetilde{\mathcal{E}_{X}^{i}}$ de l'ensemble $\mathcal{E}_{X}^{i}$ du théorème 2.6. Pour de tels plans $P$ génériques, il existe un seul domaine $\mathcal{K}^{P}$ au-dessus duquel la caractéristique d'Euler-Poincaré typique $\chi^{P}=$ $\chi\left(\pi_{P}^{-1}(y) \cap X \cap B_{(0, r)}^{2 n}\right)$ ne dépend ni de $y$, générique dans $P \backslash\{0\}$ et suffisamment proche de 0 , ni du choix du représentant $X$ de $X_{0}$, c'est-à-dire du choix de $r>0$, lorsque celui-ci est suffisamment petit. De plus $\chi^{P}$ ne dépend pas du choix de $P$, lorsque $P$ est général. Ceci résulte des mêmes arguments que dans le cas réel et du fait que le complémentaire d'un ensemble analytique complexe de codimension plus grande que 1 est connexe. Notons $\widetilde{\sigma_{i}}\left(X_{0}\right)$ l'invariant polaire complexe d'ordre $i$. Comme $\Theta_{2 i}\left(\mathcal{K}^{P}\right)=1$, on obtient :

$$
\widetilde{\sigma_{i}}\left(X_{0}\right)=\chi\left(\pi_{P}^{-1}(y) \cap X \cap B_{(0, r)}^{2 n}\right)
$$

pour $y \in P$ générique suffisamment proche de l'origine et $r>0$ suffisamment petit.

Dans le cas particulier important où $X$ est l'hypersurface $f^{-1}(0)$, donnée par une application analytique $f: \mathbb{C}^{n} \rightarrow \mathbb{C}$ admettant en 0 une singularité isolée, on a pour $y$ générique dans $\left(\mathbb{C}^{i}, 0\right)$ :

$$
\chi\left(\pi_{P}^{-1}(y) \cap X \cap B_{(0, r)}^{2 n}\right)=\chi\left(\pi_{P}^{-1}(0) \cap f^{-1}(\epsilon) \cap B_{(0, r)}^{2 n}\right),
$$

où $\epsilon$ est générique dans $\mathbb{C}$ et suffisamment proche de 0 . Ceci résulte classiquement du premier lemme d'isotopie de Thom-Mather, de la généricité de la transversalité en l'origine, pour des $(n-i)$-plans affines de $\mathbb{C}^{n}$, aux strates d'une stratification de Whitney du germe $X_{0}$ et de la connexité du complémentaire dans $\mathbb{C}^{n}$ d'un ensemble analytique complexe négligeable. Dans ce cas $\left.\chi\left(\pi_{P}^{-1}(0) \cap f^{-1}(\epsilon) \cap B_{(0, r)}^{2 n}\right)\right)$ est la caractéristique d'Euler-Poincaré de la fibre de Milnor de $f$ dans $P^{\perp}$. C'est-à-dire : $1+(-1)^{n-i-1} \mu_{n-i}$, où $\mu_{n-i}$ est le nombre de Milnor 
de la section $(n-i)$-plane de $X_{0}$ (introduit dans [73]). On a donc, dans le cas où $X_{0}$ est le germe d'une hypersurface complexe de $\mathbb{C}^{n}$ :

$$
\widetilde{\sigma}_{i}\left(X_{0}\right)=1+(-1)^{n-i-1} \mu_{n-i} .
$$

Dans [73] il est montré que l'indépendance de la suite $\left(\mu_{0}\left(X_{t}\right), \cdots, \mu_{n}\left(X_{t}\right)\right)$ relativement aux paramètres $t$ (et donc, dans ce cadre, de la suite $\left(\widetilde{\sigma}_{0}\left(X_{t}\right), \cdots, \widetilde{\sigma}_{n}\left(X_{t}\right)\right)$ ), pour une famille analytique $X=\left(X_{t}\right)_{t \in \mathbb{C}} \subset \mathbb{C}^{n+1}$ de germes d'hypersurfaces analytiques de $\mathbb{C}^{n}$ ayant 0 pour singularité isolée implique la condition de Whitney, au voisinage de 0 dans $\mathbb{C}$, pour le couple $(X \backslash \mathbb{C}, \mathbb{C})$. Et d'après [5], l'implication réciproque est vraie.

En toute généralité ( $X$ analytique complexe dans $\mathbb{C}^{n}$ de dimension quelconque et non plus seulement une hypersurface) les invariants complexes $\widetilde{\sigma}_{i}\left(X_{0}\right)$ ont été considérés pour la première fois par M. Kashiwara dans [37] (où les boules sont ouvertes et non fermées comme c'est le cas ici) : un invariant $E_{X_{0}}^{0}$ y est défini par récurrence sur la dimension de $X_{0}$ à l'aide de $\widetilde{\sigma}_{i}$. L'étude de cet invariant est reprise par A. Dubson ([Du1,2]) puis dans [8] où les auteurs en donnent une version multidimensionnelle $E_{X_{0}}^{q}$. Leur définition est la suivante :

$$
E_{X_{0}}^{k}=\sum_{X^{j_{0}} \subset \bar{X}^{j} \backslash X^{j}, \operatorname{dim}\left(X^{j}\right)<\operatorname{dim}\left(X_{0}\right)} E_{\bar{X}^{j}}^{k} \cdot \widetilde{\sigma}_{k+\operatorname{dim}\left(X^{j}\right)+1}\left(X_{0}\right),
$$

où $\left(X^{j}\right)$ est une stratification de Whitney de $X_{0}$, et $X^{j_{0}}$ la strate contenant 0 . Les auteurs remarquent ensuite (cf. aussi [Du1,2]) que :

$$
E_{X_{0}}^{k}=E u_{X_{0}}
$$

où $E u_{X_{0}}$ est l'obstruction d'Euler locale de $X$ en 0, introduite par R. MacPherson dans [53], et :

$$
(-1)^{k}\left(E_{X_{0}}^{\operatorname{dim}\left(X_{0}\right)-k-1}-E_{X_{0}}^{\operatorname{dim}\left(X_{0}\right)-k}\right)=e\left(\mathcal{P}^{k}\left(X_{0}\right), 0\right),
$$

où $e\left(\mathcal{P}^{k}\left(X_{0}\right), 0\right)$ est la multiplicité en 0 de la variété polaire $\mathcal{P}^{k}\left(X_{0}\right)$ de codimension $k$ de $X_{0}$ en 0 (voir aussi [56], [76], [77], [78], [17]). Il est annoncé sans preuve dans [16, Proposition 1], [17, Théorème II.2.7, p. 30] et [8], que les invariants $\widetilde{\sigma}_{i}\left(X_{y}\right)$ sont constants lorsque $y$ varie dans une strate d'une stratification de Whitney de $X_{0}$. Mais dans [31], [58], [74] il est prouvé que la constance des multiplicités $e\left(\mathcal{P}^{k}\left(X_{y}\right), y\right)$ lorsque $y$ varie dans une strate d'une stratification donnée de $X_{0}$ équivaut à la $(b)$-régularité de cette stratification, ce qui donne une preuve, compte tenu de l'égalité ci-dessus reliant les $e\left(\mathcal{P}^{k}\left(X_{y}\right), y\right)$ et les $\widetilde{\sigma}_{i}\left(X_{y}\right)$, de la constance des $\widetilde{\sigma}_{i}\left(X_{y}\right)$ le long de strates de Whitney.

On rassemble ces résultats dans le théorème suivant, où $e\left(\Delta^{k}\left(X_{y}\right), y\right)$ est la multiplicité en $y$ du discriminant $\Delta^{k}\left(X_{y}\right)$ associé à $\mathcal{P}^{k}\left(X_{y}\right)$.

THÉORÈMe 2.10 ([31], [58], [78], [74]). - Soit $X_{0}$ un germe en 0 d'ensemble analytique complexe de $\mathbb{C}^{n}$ muni d'une stratification $\left(X^{j}\right)$. Les conditions suivantes sont équivalentes :

(i) La stratification $\left(X^{j}\right)$ est de Whitney.

(ii) Les fonctions $y \mapsto e\left(\mathcal{P}^{k}\left(X_{y}\right), y\right)$ en restriction aux strates $X^{j}$ sont constantes.

(iii) Les fonctions $y \mapsto e\left(\Delta^{k}\left(X_{y}\right)\right.$, y) en restriction aux strates $X^{j}$ sont constantes.

(iv) Les fonctions $y \mapsto \widetilde{\sigma}_{i}\left(X_{y}\right)$ en restriction aux strates $X^{j}$ sont constantes. 
Nous donnons une preuve directe de (i) $\Rightarrow$ (iv) au corollaire 4.5, c'est-à-dire sans utiliser (i) $\Rightarrow$ (ii) et le lien entre $e\left(\mathcal{P}^{k}\left(X_{y}\right), y\right)$ et $\sigma_{i}\left(X_{y}\right)$ (la preuve de (i) $\Rightarrow$ (iv) est faite dans [5] dans le cas des hypersurfaces à singularité isolée).

\section{Courbures de Lipschitz-Killing locales et invariants polaires}

L'objet de cette section est de prouver le théorème 3.1 qui relie, via la suite $\sigma_{*}\left(X_{0}\right)$, les courbures $\Lambda_{i}^{\text {loc }}$ à la géométrie des discriminants généraux des projections du germe $X_{0}$ sur des plans de dimension $i, i+1, \ldots, n$. La preuve se fait en deux étapes. Tout d'abord pour les cônes sous-analytiques de sommet l'origine (Section 3.1), ce qui permet, par déformation sur le cône tangent, d'établir le théorème pour les germes d'ensembles sous-analytiques fermés quelconques (Section 3.2).

ThÉorème 3.1. - Pour tout $i \in\{1, \ldots, n\}$, il existe des constantes réelles $m_{i}^{i}, \ldots, m_{i}^{n}$ telles que pour tout germe $X_{0}$ d'ensemble sous-analytique fermé de $\mathbb{R}^{n}$, on ait l'égalité:

$$
\Lambda_{i}^{\mathrm{loc}}\left(X_{0}\right)=\sum_{j=i}^{n} m_{i}^{j} \cdot \sigma_{j}\left(X_{0}\right) .
$$

Autrement dit il existe une matrice triangulaire supérieure $\left(m_{i}^{j}\right)_{1 \leq i \leq n, 1 \leq j \leq n}$ telle que :

$$
\left(\begin{array}{c}
\Lambda_{1}^{\text {loc }} \\
\vdots \\
\Lambda_{n}^{\text {loc }}
\end{array}\right)=\left(\begin{array}{ccccc}
m_{1}^{1} & m_{1}^{2} & \ldots & m_{1}^{n-1} & m_{1}^{n} \\
0 & m_{2}^{2} & \ldots & m_{2}^{n-1} & m_{2}^{n} \\
\vdots & & & & \vdots \\
0 & 0 & \ldots & 0 & m_{n}^{n}
\end{array}\right) \cdot\left(\begin{array}{c}
\sigma_{1} \\
\vdots \\
\sigma_{n}
\end{array}\right)
$$

De plus : $m_{i}^{i}=1, m_{i}^{j}=\frac{\alpha_{j}}{\alpha_{j-i} \cdot \alpha_{i}} C_{j}^{i}-\frac{\alpha_{j-1}}{\alpha_{j-1-i} \cdot \alpha_{i}} C_{j-1}^{i}, \quad$ si $i+1 \leq j \leq n$.

\subsection{Le cas conique}

Soit $X_{0}$ un cône sous-analytique fermé de sommet l'origine dans $\mathbb{R}^{n}$, c'est-à-dire qu'existe $L$ un sous-analytique compact de la sphère unité de $\mathbb{R}^{n}$ tel que $: X_{0}=\mathbb{R}_{+} \cdot L$. On suppose que $\left(X^{j}\right)_{j \in\{0, \cdots, 2 k\}}$ est une stratification de Whitney de $X=X_{0} \cap B_{(0,1)}$ provenant de $L$, c'est-àdire que $\left(X^{j}\right)_{j \in\{k+1, \cdots, 2 k\}}$ est une stratification de Whitney de $L$, et que pour $j \in\{1, \cdots, k\}$, $X^{j}=\mathbb{R}_{+}^{*} \cdot X^{j+k}, X^{0}=\{0\}$. Puisque $X_{0}$ est un cône :

$$
\Lambda_{i}^{\text {loc }}\left(X_{0}\right)=\frac{1}{\alpha_{i}} \Lambda_{i}(X)
$$

On commence par rappeler le calcul fait dans [6], section 5. Pour cela on reprend les notations de [6]. Soient $Y \subset \mathbb{R}^{n}$ un ensemble sous-analytique compact, $\left(Y^{j}\right)_{j \in\{1, \cdots, k\}}$ une stratification de Whitney de $Y$ et $\nu \in \mathbb{R}^{n}$. On note :

$$
\gamma(Y, \nu, x)=1-\chi\left(B(x, \delta) \cap g_{\nu}^{-1}\left(g_{\nu}(x)-\epsilon\right)\right),
$$

pour $0<\epsilon \ll \delta \ll 1, g_{\nu}: Y \rightarrow \mathbb{R}$ où $g_{\nu}(x)=\|\nu-x\|^{2}$ et $x \in Y^{j}$ est un point critique de $g_{\nu \mid Y^{j}}$. On note encore :

$$
\gamma_{t a n}(Y, \nu, x)=1-\chi\left(B(x, \delta) \cap g_{\nu \mid Y^{j}}^{-1}\left(g_{\nu}(x)-\epsilon\right)\right)
$$


pour $0<\epsilon \ll \delta \ll 1$ et $x \in Y^{j}$ un point critique de $g_{\nu \mid Y^{j}}$ et enfin :

$$
\gamma_{\text {nor }}(Y, \nu, x)=1-\chi\left(B(x, \delta) \cap g_{\nu \mid Y \cap S}^{-1}\left(g_{\nu}(x)-\epsilon\right)\right),
$$

pour $0<\epsilon \ll \delta \ll 1$ et $x \in Y^{j}$ un point critique de $g_{\nu \mid Y^{j} \cap S}$, où $S$ est une variété lisse, de dimension $n-\operatorname{dim}\left(Y^{j}\right)$, transverse à $Y^{j}$ en $x$. On a alors ([26]) :

$$
\gamma(Y, \nu, x)=\gamma_{\tan }(Y, \nu, x) \cdot \gamma_{\text {nor }}(Y, \nu, x),
$$

et pour presque tout $\nu \in \mathbb{R}^{n}$ ([6, Lemme 3.5]), en convenant que $\gamma(Y, \nu, x)=0$ lorsque $\gamma(Y, \nu, x)$ n'est pas défini, c'est-à-dire lorsque $x$ est non critique pour $g_{\nu \mid Y^{j}}$ :

$$
\chi(Y)=\sum_{x \in Y} \gamma(Y, \nu, x)
$$

Notons $\pi: \mathcal{N} \rightarrow X^{j}$ le fibré normal d'une strate $X^{j}$ de $X, \mathcal{N}_{1}$ son fibré normal unitaire et pour $r \in \mathbb{R}, \mathcal{N}_{r}=\left\{(t, \nu, x) \in \mathbb{R}_{+} \times \mathcal{N}_{1} \subset \mathbb{R}_{+} \times \mathbb{R}^{n} \times X^{j} ;\|\nu\| \leq r\right\}$; enfin soit $\Psi: \mathcal{N} \rightarrow \mathbb{R}^{n}$ l'application définie par $\Psi((t, \nu, x))=x+t \cdot \nu$.

On pose :

$$
\Lambda\left(X, X^{j}\right)(x, r)=\int_{t \leq r} \int_{\nu \in \pi^{-1}(x) \cap \mathcal{N}_{1}} \gamma_{\mathrm{nor}}(X, \nu, x) \cdot \phi(t, \nu, x) d t d \nu
$$

où $\phi$ est $J a c(\Psi)$. On va calculer :

$$
\sum_{j=0}^{2 k} \int_{x \in X^{j}} \Lambda\left(X, X^{j}\right)(x, r) d x .
$$

Pour cela remarquons que si $d=\operatorname{dim}\left(X^{j}\right)$ :

$$
\phi(t, \nu, x)=\operatorname{det}\left(\begin{array}{ccc}
1 & 0 & 0 \\
* t \cdot I_{n-d-1} & 0 \\
* & * & I_{d}+t \cdot \operatorname{II}(\nu, x)
\end{array}\right),
$$

où $I_{d}$ est la matrice unité d'ordre $d$ et $\mathrm{II}(\nu, x)=\mathrm{II}_{X^{j}}(\nu, x)$ la matrice dans une base orthonormée de $T_{x} X^{j}$ de la seconde forme fondamentale de $X^{j}$ en $x$ suivant la direction normale $\nu$. La forme $\operatorname{II}(\nu, x)$ est définie de la façon suivante : si $\mu(s)$ et $\tau(s)$ sont deux chemins différentiables tracés sur $X^{j}$, tels que $\mu(0)=\tau(0)=x$ et si $\nu(y)$ est un champ de vecteurs normaux à $X^{j}$, tel que $\nu(x)=\nu$ et si d'autre part $x=x\left(u_{1}, \cdots, u_{d}\right)$ sont des coordonnées sur $X^{j}$ telles que $x=x(0, \cdots, 0), \frac{\partial x}{\partial u_{i}}(0)=\mu^{\prime}(0), \frac{\partial x}{\partial u_{j}}(0)=\tau^{\prime}(0)$ :

$$
\mathrm{II}(\nu, x)\left(\mu^{\prime}(0), \tau^{\prime}(0)\right)=\left\langle D \nu_{(x)}\left(\mu^{\prime}(0)\right) \mid \tau^{\prime}(0)\right\rangle=-\left\langle\nu \mid \frac{\partial^{2} x}{\partial u_{i} \partial u_{j}}(0)\right\rangle .
$$

Les propriétés classiques de finitude en géométrie modérée montrent alors que $\Lambda\left(X, X^{j}\right)(x, r)$ est un polynôme en $r$ que l'on écrit :

$$
\Lambda\left(X, X^{j}\right)(x, r)=\sum_{i=n-d}^{n} \lambda_{n-i}\left(X, X^{j}\right)(x) \cdot \alpha_{i} \cdot r^{i} .
$$


En observant que le signe de $\phi(t, \nu, x)$ est $\gamma_{\tan }(X, t . \nu, x)$ (cf. [6, 5.1.4]) et $\gamma_{\text {nor }}(X, \nu, x)=$ $\gamma_{\text {nor }}(X, t . \nu, x)$, on déduit que :

$$
\begin{gathered}
\int_{x \in X^{j}} \Lambda\left(X, X^{j}\right)(x, r) d x=\sum_{i=n-d}^{n} \alpha_{i} \cdot \int_{x \in X^{j}} \lambda_{n-i}\left(X, X^{j}\right)(x) d x \cdot r^{i}= \\
\int_{y \in \mathbb{R}^{n}} \sum_{x \in X^{j},\|x-y\| \leq r} \gamma(X, y, x) d y .
\end{gathered}
$$

Puis en sommant sur toutes les strates et en convenant que $\lambda_{\ell}\left(X, X^{j}\right)=0$ si $\ell>\operatorname{dim}\left(X^{j}\right)$ :

$$
\begin{gathered}
\sum_{j=0}^{2 k} \int_{x \in X^{j}} \Lambda\left(X, X^{j}\right)(x, r) d x=\sum_{i=0}^{n} \alpha_{i}\left(\sum_{j=0}^{2 k} \int_{x \in X^{j}} \lambda_{n-i}\left(X, X^{j}\right)(x) d x\right) \cdot r^{i}= \\
\int_{y \in \mathbb{R}^{n}} \sum_{x \in X,\|x-y\| \leq r} \gamma(X, y, x) d y=\int_{y \in \mathbb{R}^{n}} \chi(X \cap B(y, r)) d y .
\end{gathered}
$$

On conclut de ce calcul que :

$$
\Lambda_{i}^{\mathrm{loc}}\left(X_{0}\right)=\frac{1}{\alpha_{i}} \Lambda_{i}(X)=\frac{1}{\alpha_{i}} \sum_{j=0}^{2 k} \int_{x \in X^{j}} \lambda_{i}\left(X, X^{j}\right)(x) d x .
$$

Dans cette somme seules les strates $X^{j}$ de dimension plus grandes que $i$ interviennent effectivement. De la même façon, en faisant jouer à $L=X \cap S_{(0,1)}^{n-1}$ et à sa stratification $\left(X^{j}\right)_{j \in\{k+1, \cdots, 2 k\}}$ le rôle que viennent de jouer $X$ et $\left(X^{j}\right)_{j \in\{0, \cdots, 2 k\}}$, on obtient :

$$
\Lambda_{i}(L)=\frac{1}{\alpha_{i}} \sum_{j=k+1}^{2 k} \int_{x \in X^{j}} \lambda_{i}\left(L, X^{j}\right)(x) d x
$$

là encore, seules les strates de dimension plus grande que $i$ ont une contribution non nulle.

On montre maintenant que tout comme $\Lambda_{i}(L)$, on peut exprimer $\Lambda_{i}^{\text {loc }}\left(X_{0}\right)$ comme une combinaison linéaire à coefficients universels des seules quantités $\int_{x \in X^{j}} \lambda_{i}\left(L, X^{j}\right)(x) d x$, pour $j \in\{k+1, \cdots, 2 k\}$. Ce qui permet d'exprimer $\Lambda_{i}^{\text {loc }}\left(X_{0}\right)$ comme combinaison linéaire des $\Lambda_{j}(L)$. Ceci est possible grâce au caractère conique de $X$ et montre que les invariants introduits dans [2] sont des combinaisons linéaires des $\Lambda_{i}^{\text {loc }}$ (cf. [2, Remarque 5.4]), dans un premier temps pour les cônes sous-analytiques, puis d'après la section 3.2 qui suit, dans le cas le plus général.

Soient $j \in\{1, \cdots, k\}, X^{j}$ une strate conique de dimension $d$ et $x \in X^{j}$. Alors $\left.\|x\| \in\right] 0,1[$ et $r(x)=x /\|x\| \in X^{j+k}$. Pour $\nu$ un vecteur unitaire normal à $X^{j}$ en $x$, et pour $\theta \in[0, \pi]$, on note $\nu_{\theta}=\sin (\theta) \cdot \nu+\cos (\theta) \cdot r(x)$, un vecteur unitaire normal à $X^{j+k}$ en $r(x)$. Lorsque $\nu$ décrit $\pi^{-1}(\{x\}) \cap \mathcal{N}_{1}$ et $\theta$ décrit $[0, \pi], \nu_{\theta}$ décrit $\pi^{-1}(\{r(x)\}) \cap \mathcal{N}_{1}$. On remarque alors que :

$$
\left.\gamma_{\text {nor }}(X, \nu, x)=\gamma_{\text {nor }}(X, \nu, u \cdot r(x))=\gamma_{\text {nor }}\left(L, \nu_{\theta}, r(x)\right), \forall u \in\right] 0,1[, \forall \theta \in] 0, \pi[.
$$

La première égalité résulte directement de la structure conique de $X$, la seconde s'obtient en faisant jouer le rôle de $S$ à la sphère $S(0,1)$ dans la définition de $\gamma_{\text {nor }}(X, \nu, u \cdot r(x))$.

Puis on remarque que :

$$
\left.\gamma_{\text {nor }}(X, \nu, x)=\gamma_{\text {nor }}\left(X, \nu_{\theta}, r(x)\right), \forall \theta \in\right] 0, \frac{\pi}{2}[
$$


car $\nu_{\theta}$ attaché à $r(x)$ pointe en dehors de $B(0,1)$, pour $\left.\theta \in\right] 0, \frac{\pi}{2}[$, tandis que toujours du fait de la structure conique de $X$ et du fait que $\nu_{\theta}$ attaché à $r(x)$ pointe dans $B(0,1)$, pour $\theta \in] \frac{\pi}{2}, \pi[:$

$$
\left.\gamma_{\text {nor }}\left(X, \nu_{\theta}, r(x)\right)=0, \forall \theta \in\right] \frac{\pi}{2}, \pi[.
$$

Fixons $\theta \in[0, \pi]$ et comparons maintenant $\mathrm{II}_{X^{j}}(\nu, x)$ et $\mathrm{II}_{X^{j+k}}\left(\nu_{\theta}, r(x)\right)$. Suivant la direction $r(x), D \nu_{(x)}$ est nulle du fait de la structure conique de $X^{j}$, d'autre part si $\nu_{\theta}$ est un champ de vecteurs unitaires normaux à $X^{j+k}$, écrit sous la forme :

$$
X^{j} \ni y \mapsto \nu_{\theta}(r(y))=\sin (\theta) . \nu(y)+\cos (\theta) . r(y),
$$

$X^{j} \ni y \mapsto \nu(\|x\| . y)$ est un champ de vecteurs unitaires normaux à $X^{j}$ en $\|x\| . y$, et si $\hat{\mu}$ est un chemin tracé dans $X^{j+k}$ passant par $r(x)$ en 0 et tel que $\hat{\mu}^{\prime}(0) \in T_{(r(x))} X^{j+k}$ est unitaire, $\mu=\|x\| \hat{\mu}$ est un chemin tracé dans $X^{j}$, passant par $x$ en 0 et $\mu^{\prime}(0) \in T_{x} X^{j}$ est de norme $\|x\|$. On a alors, puisque :

$$
\begin{gathered}
\nu_{\theta} \circ \hat{\mu}=\sin (\theta) . \nu \circ \mu+\cos (\theta) . r \circ \mu \\
D \nu_{\theta[r(x)]}\left(\hat{\mu}^{\prime}(0)\right)=\sin (\theta) \cdot D \nu_{(x)}\left(\mu^{\prime}(0)\right)+\cos (\theta) \cdot D r_{(x)}\left(\mu^{\prime}(0)\right) \\
{ }^{t}\left[\hat{\mu}^{\prime}(0)\right] \cdot \mathrm{II}_{X^{j+k}}\left(\nu_{\theta}, r(x)\right)=\sin (\theta) \cdot\|x\| \cdot{ }^{t}\left[\hat{\mu}^{\prime}(0)\right] \cdot \mathrm{II}_{X^{j}}(\nu, x) \\
+\cos (\theta) \cdot\|x\| \cdot{ }^{t}\left[\hat{\mu}^{\prime}(0)\right] \cdot \mathrm{II}_{S_{(0,\|x\|)}^{n-1}}(r(x), x)_{\mid T_{x} X^{j}} \\
\mathrm{II}_{X^{j+k}}\left(\nu_{\theta}, r(x)\right)=\sin (\theta) \cdot\|x\| \cdot \mathrm{II}_{X^{j}}(\nu, x)+\cos (\theta) \cdot I_{d-1} .
\end{gathered}
$$

On note $A$ et $C$ les matrices d'ordre $d-1$ définies par les relations suivantes :

$$
\mathrm{II}_{X^{j}}(\nu, x)=\left(\begin{array}{ll}
0 & 0 \\
0 & A
\end{array}\right), \quad C=\|x\| \cdot A .
$$

On a alors d'une part :

$$
\begin{gathered}
\Lambda\left(X, X^{j}\right)(x, r)=\int_{t \leq r} \int_{\nu \in \pi^{-1}(x) \cap \mathcal{N}_{1}} \gamma_{\text {nor }}(X, \nu, x) \cdot \phi(t, \nu, x) d t d \nu \\
=\int_{t \leq r} \int_{\nu \in \pi^{-1}(x) \cap \mathcal{N}_{1}} \gamma_{\text {nor }}(X, \nu, x) \cdot \operatorname{det}\left(\begin{array}{cccc}
1 & 0 & 0 & 0 \\
* t \cdot I_{n-d-1} & 0 & 0 \\
* & * & 1 & 0 \\
* & * & * I_{d-1}+t \cdot A
\end{array}\right) d t d \nu \\
\text { (1) }=\int_{t \leq r} \int_{\nu \in \pi^{-1}(x) \cap \mathcal{N}_{1}} \gamma_{\text {nor }}(X, \nu, x) \cdot \operatorname{det}\left(\begin{array}{cccc}
1 & 0 & 0 & 0 \\
* t \cdot I_{n-d-1} & 0 & 0 \\
* & * & 1 & 0 \\
* & * & * I_{d-1}+t \cdot \frac{C}{\|x\|}
\end{array}\right) d t d \nu .
\end{gathered}
$$

Et d'autre part, d'après $(a)$ et $(b)$ :

$$
\begin{aligned}
\Lambda\left(X, X^{j+k}\right)(r(x), r)= & \int_{t \leq r} \int_{\nu_{\theta} \in \pi^{-1}(r(x)) \cap \mathcal{N}_{1}} \gamma_{\text {nor }}\left(X, \nu_{\theta}, r(x)\right) \cdot \phi\left(t, \nu_{\theta}, r(x)\right) d t d \nu_{\theta} \\
& =\int_{t \leq r} \int_{u \in \pi^{-1}(x) \cap \mathcal{N}_{1}} \gamma_{\text {nor }}(X, \nu, x)
\end{aligned}
$$


(2) $\quad \int_{\theta \in\left[0, \frac{\pi}{2}\right]} f(\theta) \cdot \operatorname{det}\left(\begin{array}{ccc}1 & 0 & 0 \\ * t \cdot I_{n-d} & 0 \\ * & * & I_{d-1}+t \cdot\left(\sin (\theta) \cdot C+\cos (\theta) \cdot I_{d-1}\right)\end{array}\right) d t d \nu d \theta$,

avec $f(\theta)=\sin ^{n-d-1}(\theta)$. Notons :

$$
\operatorname{det}\left(C+T \cdot I_{d-1}\right)=\sum_{p=0}^{d-1} a_{p} T^{p}, \quad\left(a_{p}=a_{p}(r(x), \nu)\right) .
$$

D'après (1) :

$$
\begin{gathered}
\Lambda\left(X, X^{j}\right)(x, r)=\int_{t \leq r} \int_{\nu \in \pi^{-1}(x) \cap \mathcal{N}_{1}} \gamma_{\text {nor }}(X, \nu, x) \cdot t^{n-d-1} \cdot \operatorname{det}\left(I_{d-1}+\frac{t}{\|x\|} \cdot C\right) d t d \nu \\
\Lambda\left(X, X^{j}\right)(x, r)=\sum_{p=0}^{d-1} \int_{t \leq r} \int_{\nu \in \pi^{-1}(x) \cap \mathcal{N}_{1}} \frac{\gamma_{\text {nor }}(X, \nu, x)}{\|x\|^{d-1-p}} \cdot a_{p} \cdot t^{n-2-p} d t d \nu \\
\Lambda\left(X, X^{j}\right)(x, r)=\sum_{p=0}^{d-1} \frac{r^{n-1-p}}{n-p-1} \cdot \int_{\nu \in \pi^{-1}(x) \cap \mathcal{N}_{1}} a_{p} \frac{\gamma_{\text {nor }}(X, \nu, x)}{\|x\|^{d-1-p}} d \nu .
\end{gathered}
$$

Soit :

$$
\int_{x \in X^{j}} \Lambda\left(X, X^{j}\right)(x, r) d x=\sum_{i=n-d}^{n-1} \Gamma_{n-i-1}(X) \cdot \frac{K_{n, i}}{i} \cdot r^{i}
$$

où

$$
\Gamma_{p}(X)=\int_{r(x) \in X^{j+k}} \int_{\nu \in \pi^{-1}(x) \cap \mathcal{N}_{1}} a_{p} \cdot \gamma_{\text {nor }}(X, \nu, r(x)) d \nu d r(x)
$$

et où $K_{n, i}$ est une constante ne dépendant que de $n$ et $i$ et qui provient de :

$$
\int_{x \in X^{j}} \int_{\nu \in \pi^{-1}(x) \cap \mathcal{N}_{1}} a_{i} \frac{\gamma_{\text {nor }}(X, \nu, x)}{\|x\|^{d+i-n}} d \nu d x=K_{n, i} \cdot \Gamma_{i}(X) .
$$

Notons que l'indépendance de $K_{n, i}$ relativement à $d$ vient du fait que $X^{j}$ est de dimension $d$ et que l'on calcule $\int_{x \in X^{j}} \int_{\nu \in \pi^{-1}(x) \cap \mathcal{N}_{1}} a_{i} \frac{\gamma_{\text {nor }}(X, \nu, x)}{\|x\|^{d+i-n}} d \nu d x$ grâce au changement de variables $\left.X^{j+k} \times\right] 0,1\left[\ni(r(x), t) \mapsto t \cdot r(x) \in X^{j}\right.$. L'égalité (3) ensuite donne :

$\int_{x \in X^{j}} \Lambda\left(X, X^{j}\right)(x, r) d x=\sum_{i=n-d}^{n-1} \Gamma_{n-i-1}(X) \cdot \frac{K_{n, i}}{i} \cdot r^{i}=\sum_{i=n-d}^{n} \int_{x \in X^{j}} \lambda_{n-i}\left(X, X^{j}\right) \cdot \alpha_{i} \cdot r^{i}$,

et donc :

$$
\int_{x \in X^{j}} \lambda_{0}\left(X, X^{j}\right) d x=0
$$

(4) $\int_{x \in X^{j}} \lambda_{p+1}\left(X, X^{j}\right) d x=\Gamma_{p}(X) \cdot \frac{K_{n, n-p-1}}{(n-p-1) \cdot \alpha_{n-p-1}}, p \in\{0, \cdots, d-1\}$.

Maintenant, d'après (2) :

$$
\begin{gathered}
\Lambda\left(X, X^{j+k}\right)(r(x), r)=\int_{t \leq r} \int_{\nu \in \pi^{-1}(x) \cap \mathcal{N}_{1}} \gamma_{\text {nor }}(X, \nu, x) \\
\int_{\theta \in\left[0, \frac{\pi}{2}\right]} f(\theta) \cdot \sin ^{d-1}(\theta) \cdot t^{n-1} \cdot \operatorname{det}\left(C+\left(\frac{1}{t \sin (\theta)}+\frac{\cos (\theta)}{\sin (\theta)}\right) \cdot I_{d-1}\right) d \theta d \nu d t .
\end{gathered}
$$




\section{Posons :}

$\alpha=\alpha(\theta)=1 / \sin (\theta), \quad \beta=\beta(\theta)=\cos (\theta) / \sin (\theta)$ et $g(\theta)=\sin ^{d-1}(\theta) \cdot f(\theta)=\sin ^{n-2}(\theta)$.

On obtient :

$$
\begin{aligned}
\Lambda\left(X, X^{j+k}\right)(r(x), r)= & \int_{t \leq r} \int_{\nu \in \pi^{-1}(x) \cap \mathcal{N}_{1}} \gamma_{\text {nor }}(X, \nu, x) \\
& \times \int_{\theta \in\left[0, \frac{\pi}{2}\right]} g(\theta) \cdot t^{n-1} \cdot \sum_{p=0}^{d-1} a_{p} \cdot\left(\frac{\alpha}{t}+\beta\right)^{p} d \theta d \nu d t \\
\Lambda\left(X, X^{j+k}\right)(r(x), r)= & \int_{t \leq r} \int_{\nu \in \pi^{-1}(x) \cap \mathcal{N}_{1}} \gamma_{\text {nor }}(X, \nu, x) d \nu \\
& \times \sum_{q=0}^{d-1} t^{n-q-1} \int_{\theta \in\left[0, \frac{\pi}{2}\right]} g(\theta) \cdot \alpha^{q} \sum_{p=q}^{d-1} a_{p} \cdot C_{p}^{q} \cdot \beta^{p-q} d \theta d t \\
\text { (1) } \int_{r(x) \in X^{j}} \Lambda\left(X, X^{j+k}\right)(r(x), r) & d r(x)=\sum_{i=n-d+1}^{n} \frac{r^{i}}{i} \sum_{p=n-i}^{d-1} \Gamma_{p}(X) \cdot \delta(i, p),
\end{aligned}
$$

où :

$$
\delta(i, p)=\int_{\theta \in\left[0, \frac{\pi}{2}\right]} g(\theta) \cdot \alpha^{n-i} \cdot C_{p}^{n-i} \cdot \beta^{p-n+i} d \theta .
$$

Notons que les $\delta(i, p)$ sont bien définis, car du fait que nos invariants sont intrinsèques, on peut toujours supposer que $d<n$, quitte à voir $X$ dans $\mathbb{R}^{n+1}$, au lieu de $\mathbb{R}^{n}$. L'égalité (5) donne alors :

$$
\sum_{i=n-d+1}^{n} \int_{r(x) \in X^{j}} \lambda_{n-i}\left(X, X^{j+k}\right) d r(x) \cdot \alpha_{i} \cdot r^{i}=\sum_{i=n-d+1}^{n} \frac{r^{i}}{i} \sum_{p=n-i}^{d-1} \Gamma_{p}(X) \cdot \delta(i, p)
$$

soit :

$$
\int_{r(x) \in X^{j}} \lambda_{n-i}\left(X, X^{j+k}\right) d r(x)=\frac{1}{i \cdot \alpha_{i}} \sum_{p=n-i}^{d-1} \Gamma_{p}(X) \cdot \delta(i, p),
$$

et d'après (4) :

$$
\begin{aligned}
& \int_{r(x) \in X^{j+k}} \lambda_{n-i}\left(X, X^{j+k}\right) d r(x) \\
&=\frac{1}{i \cdot \alpha_{i}} \sum_{p=n-i}^{d-1} \frac{(n-p-1) \cdot \alpha_{n-p-1}}{K_{n, n-p-1}} \cdot \delta(i, p) \int_{x \in X^{j}} \lambda_{p+1}\left(X, X^{j}\right) d x \\
& \times \int_{r(x) \in X^{j+k}} \lambda_{\ell}\left(X, X^{j+k}\right) d r(x) \\
&= \sum_{p=\ell}^{d-1} \frac{(n-p-1) \cdot \alpha_{n-p-1}}{(n-\ell) \cdot \alpha_{n-\ell} \cdot K_{n, n-p-1}} \cdot \delta(n-\ell, p) \int_{x \in X^{j}} \lambda_{p+1}\left(X, X^{j}\right) d x
\end{aligned}
$$

Soit en notant $M_{n}(\ell, p)=\frac{(n-p-1) \cdot \alpha_{n-p-1}}{(n-\ell) \cdot \alpha_{n-\ell} \cdot K_{n, n-p-1}} \cdot \delta(n-\ell, p)$ :

(6) $\int_{r(x) \in X^{j+k}} \lambda_{\ell}\left(X, X^{j+k}\right) d r(x)=\sum_{p=\ell}^{d-1} M_{n}(\ell, p) \cdot \int_{x \in X^{j}} \lambda_{p+1}\left(X, X^{j}\right) d x, \ell \in\{0, \cdots, d-1\}$. 
ou encore si :

$$
\begin{gathered}
Q=\left(Q_{n}(i, j)\right)_{i, j \in\{0, \cdots, d-1\}}=\left(\begin{array}{cccc}
M_{n}(0,0) & M_{n}(0,1) & \cdots & M_{n}(0, d-1) \\
0 & M_{n}(1,1) & \cdots & M_{n}(1, d-1) \\
\vdots & & & \vdots \\
0 & \cdots & 0 & M_{n}(d-1, d-1)
\end{array}\right) \\
\qquad \int_{x \in X^{j}} \lambda_{\ell+1}\left(X, X^{j}\right) d r(x) \\
=\sum_{p=\ell}^{d-1} Q_{n}(\ell, p) \cdot \int_{x \in X^{j+k}} \lambda_{p}\left(X, X^{j+k}\right) d x, \ell \in\{0, \cdots, d-1\} .
\end{gathered}
$$

Enfin, le même calcul montre que :

$$
\int_{r(x) \in X^{j+k}} \lambda_{n-i}\left(L, X^{j+k}\right) d r(x)=\frac{1}{i \cdot \alpha_{i}} \sum_{p=n-i}^{d-1} \Gamma_{p}(X) \cdot \widetilde{\delta}(i, p),
$$

où :

$$
\widetilde{\delta}(i, p)=\int_{\theta \in[0, \pi]} g(\theta) \cdot \alpha^{n-i} \cdot C_{p}^{n-i} \cdot \beta^{p-n+i} d \theta .
$$

Si on définit $\widetilde{M}_{n}(i, j)$ de la même façon que $M_{n}(i, j)$, mais en remplaçant $\delta(i, p) \operatorname{par} \widetilde{\delta}(i, p)$, puis si $\widetilde{Q}$ désigne l'inverse de la matrice triangulaire $\left(\widetilde{M}_{n}(i, j)\right)_{j \geq i}$, on obtient finalement :

$$
\begin{aligned}
\left(\begin{array}{c}
\int_{x \in X^{j}} \lambda_{1}\left(X, X^{j}\right) d x \\
\vdots \\
\int_{x \in X^{j}} \lambda_{d}\left(X, X^{j}\right) d x
\end{array}\right)=Q \cdot\left(\begin{array}{c}
\int_{x \in X^{j+k}} \lambda_{0}\left(X, X^{j+k}\right) d x \\
\vdots \\
\int_{x \in X^{j+k}} \lambda_{d-1}\left(X, X^{j+k}\right) d x
\end{array}\right) \\
=\widetilde{Q} \cdot\left(\begin{array}{c}
\int_{x \in X^{j+k}} \lambda_{0}\left(L, X^{j+k}\right) d x \\
\vdots \\
\int_{x \in X^{j+k}} \lambda_{d-1}\left(L, X^{j+k}\right) d x
\end{array}\right)
\end{aligned}
$$

Notons que la matrice $\widetilde{Q}=\widetilde{Q}\left(X^{j}\right)$, dont l'ordre est la dimension de $X^{j}$, ne dépend de $X^{j}$, ou plutôt de $\operatorname{dim}\left(X^{j}\right)$, que par sa taille; les coefficients de $\widetilde{Q}$ ne dépendent en effet pas de la dimension de $X^{j}$. Pour être plus précis, si pour $j, j^{\prime} \leq k, \operatorname{dim}\left(X^{j}\right) \leq \operatorname{dim}\left(X^{j^{\prime}}\right)$, on a :

$$
\widetilde{Q}\left(X^{j^{\prime}}\right)=\left(\begin{array}{r}
\widetilde{Q}\left(X^{j}\right) * \\
*
\end{array}\right)
$$

De plus comme $\lambda_{i}\left(X, X^{0}\right)=0$, pour $i \neq 0$, on a pour $i \geq 1$ :

$$
\Lambda_{i}^{\mathrm{loc}}\left(X_{0}\right)=\frac{1}{\alpha_{i}} \Lambda_{i}(X)=\frac{1}{\alpha_{i}} \sum_{j=0}^{2 k} \int_{x \in X^{j}} \lambda_{i}\left(X, X^{j}\right) d x=\frac{1}{\alpha_{i}} \sum_{j=1}^{2 k} \int_{x \in X^{j}} \lambda_{i}\left(X, X^{j}\right) d x,
$$

et en notant $\widetilde{Q}_{i}\left(X^{j}\right)$ la $i$-ème ligne de $\widetilde{Q}\left(X^{j}\right)$, on obtient : 


$$
\begin{aligned}
\Lambda_{i}^{\mathrm{loc}}\left(X_{0}\right) & =\frac{1}{\alpha_{i}} \sum_{j=1}^{2 k} \int_{x \in X^{j}} \lambda_{i}\left(X, X^{j}\right) d x \\
& =\frac{1}{\alpha_{i}}\left(\sum_{j=1}^{k} \int_{x \in X^{j}} \lambda_{i}\left(X, X^{j}\right) d x+\sum_{j=1}^{k} \int_{x \in X^{j+k}} \lambda_{i}\left(X, X^{j+k}\right) d x\right) \\
& =\frac{1}{\alpha_{i}} \sum_{j=1}^{k}\left(\widetilde{Q}_{i}\left(X^{j}\right)+\left[Q^{-1}\left(X^{j}\right) \widetilde{Q}\left(X^{j}\right)\right]_{i}\right)\left(\begin{array}{c}
\int_{x \in X^{j+k}} \lambda_{0}\left(L, X^{j+k}\right) d x \\
\vdots \\
\int_{x \in X^{j+k}} \lambda_{\operatorname{dim}\left(X^{j}\right)-1}\left(L, X^{j+k}\right) d x
\end{array}\right)
\end{aligned}
$$

Soit :

$$
\Lambda_{i}^{\mathrm{loc}}\left(X_{0}\right)=\hat{Q}_{i}\left(\begin{array}{c}
\Lambda_{0}(L) \\
\vdots \\
\Lambda_{\ell-1}(L)
\end{array}\right),
$$

avec $\operatorname{dim}(X)=\ell=\operatorname{dim}\left(X^{\hat{j}}\right), \hat{Q}$ la matrice d'ordre $\ell$ dont la $i$-ème ligne $\hat{Q}_{i}$ est $\left[\widetilde{Q}\left(X^{\hat{j}}\right)+\right.$ $\left.Q^{-1}\left(X^{\hat{j}}\right) \widetilde{Q}\left(X^{\hat{j}}\right)\right]_{i}$. Notons que $\hat{Q}$ est triangulaire supérieure et que :

$$
\left(\begin{array}{c}
\Lambda_{1}^{\mathrm{loc}}\left(X_{0}\right) \\
\vdots \\
\Lambda_{\ell}^{\mathrm{loc}}\left(X_{0}\right)
\end{array}\right)=\hat{Q}\left(\begin{array}{c}
\Lambda_{0}(L) \\
\vdots \\
\Lambda_{\ell-1}(L)
\end{array}\right) .
$$

Cette égalité est à comparer avec celle donnée dans [2, Remarque 5.4].

Exprimons maintenant $\sigma_{j}\left(X_{0}\right)$ comme combinaison linéaire à coefficients universels des invariants $\Lambda_{i}(L)$. Par définition :

$$
\sigma_{j}\left(X_{0}\right)=\int_{P \in \mathcal{E}_{X}^{j}} \frac{1}{j \cdot \alpha_{j}} \int_{\ell \in S_{(0,1)}^{P}} \frac{1}{2} \cdot\left(\chi_{-}^{\ell}+\chi_{+}^{\ell}\right) d \ell d \gamma_{j, n}(P),
$$

où $S_{(0,1)}^{P}$ est la sphère unité de $P, \pi_{P, \ell}$ la projection orthogonale de $\ell \oplus P^{\perp}$ sur $\ell$ (on identifie la direction $\ell$ et la droite qu'elle supporte) et $0<\epsilon=\epsilon(X, \ell) \ll 1, \chi_{-}^{\ell}=\chi\left(X \cap \pi_{P, \ell}^{-1}(-\epsilon)\right)$, $\chi_{+}^{\ell}=$ $\chi\left(X \cap \pi_{P, \ell}^{-1}(\epsilon)\right)$. Remarquons que du fait que $X_{0}$ est un cône, $\left.\left.\chi_{-}^{\ell}=\chi\left(L \cap \pi_{P, \ell}^{-1}(]-\infty,-\epsilon\right]\right)\right)$ et $\chi_{+}^{\ell}=\chi\left(L \cap \pi_{P, \ell}^{-1}([\epsilon,+\infty[))\right.$. Mais d'autre part, le premier lemme d'isotopie de Thom-Mather montre que :

$$
\begin{gathered}
\left.\left.\left.\left.\chi\left(L \cap \pi_{P, \ell}^{-1}(]-\infty,-\epsilon\right]\right)\right)=\chi\left(L \cap \pi_{P, \ell}^{-1}(]-\infty, 0\right]\right)\right), \\
\chi\left(L \cap \pi _ { P , \ell } ^ { - 1 } \left([\epsilon,+\infty[))=\chi\left(L \cap \pi_{P, \ell}^{-1}([0,+\infty[)),\right.\right.\right.
\end{gathered}
$$

puisque pour $\epsilon$ suffisamment proche de $0, \pi_{P, \ell}^{-1}(\epsilon)$ est transverse aux strates de $L$ (Proposition 2.5). On obtient alors :

$$
\sigma_{j}\left(X_{0}\right)=\frac{1}{j \cdot \alpha_{j}} \int_{P \in \mathcal{E}_{X}^{j}} \int_{\ell \in S_{(0,1)}^{P}} \frac{1}{2} \cdot\left(\chi\left(L \cap P^{\perp}\right)+\chi\left(L \cap \ell \oplus P^{\perp}\right)\right) d \ell d \gamma_{j, n}(P) .
$$


Il existe par conséquent deux constantes $a(j, n)$ et $a^{\prime}(j, n)$ ne dépendant que de $j$ et $n$ telles que :

$$
\sigma_{j}\left(X_{0}\right)=a(j, n) \int_{P \in \mathcal{E}_{X}^{j}} \chi\left(L \cap P^{\perp}\right) d \gamma_{j, n}(P)+a^{\prime}(j, n) \int_{H \in \mathcal{E}_{X}^{\prime n-j+1}} \chi(L \cap H) d \gamma_{j, n}(P),
$$

avec $\mathcal{E}_{X}^{\prime n-j+1}$ un sous-analytique dense de $G(n-j+1, n)$. En notant $\mathcal{O}\left(S^{n-1}\right)$ le groupe des isométries de $S_{(0,1)}^{n-1}$, il existe un sous-analytique dense $O_{X}^{j}$ dans $\mathcal{O}\left(S^{n-1}\right)$ tel que :

$$
\sigma_{j}\left(X_{0}\right)=\widetilde{a}(j, n) \int_{\sigma \in O_{X}^{j}} \chi(L \cap \sigma \cdot K) d \sigma+\widetilde{a}^{\prime}(j, n) \int_{\sigma \in O_{X}^{j}} \chi(L \cap \sigma \cdot H) d \sigma,
$$

où $K$ est la trace dans $S_{(0,1)}^{n-1}$ d'un $(n-j)$ plan vectoriel fixé et $H$ celle d'un $(n-j+1)$ plan vectoriel fixé. En utilisant $\chi=\Lambda_{0}$, puis le fait que $\Lambda_{0}$ (comme les $\Lambda_{p}$ ) est combinaison linéaire des courbures sphériques relatives $\widetilde{\Lambda}_{i}([2$, Théorème 1.1], ou formule de Gauss-Bonnet sphérique, [2, Théorème 1.2]) introduites dans [2], on obtient en notant $\ell=\operatorname{dim}(X)$ :

$$
\begin{aligned}
\sigma_{j}\left(X_{0}\right)= & \widetilde{a}(j, n) \sum_{i=0}^{\ell-1} \int_{\sigma \in O_{X}^{j}} b(i) \cdot \widetilde{\Lambda}_{i}(L \cap \sigma \cdot K) d \sigma \\
& +\widetilde{a}^{\prime}(j, n) \sum_{i=0}^{\ell-1} \int_{\sigma \in O_{X}^{j}} b(i) \cdot \widetilde{\Lambda}_{i}(L \cap \sigma \cdot H) d \sigma .
\end{aligned}
$$

La formule cinématique ([2, Théorème 4.4]) pour les courbures sphériques relatives donne ensuite :

$$
\sigma_{j}\left(X_{0}\right)=\sum_{i=j-1}^{\ell-1} c(i, j, n) \cdot \widetilde{\Lambda}_{i}(L)
$$

En utilisant encore que $\widetilde{\Lambda}_{i}$ est combinaison linéaire des $\Lambda_{p}$, pour $p \geq i$, on en déduit :

$$
\sigma_{j}\left(X_{0}\right)=\sum_{i=j-1}^{\ell-1} \hat{c}(i, j, n) \cdot \Lambda_{i}(L)
$$

En notant $R$ la matrice triangulaire supérieure dont les coefficients sont les $\hat{c}(i, j, n), j-1 \leq$ $i \leq \ell-1$ :

$$
\left(\begin{array}{c}
\sigma_{1}\left(X_{0}\right) \\
\vdots \\
\sigma_{\ell}\left(X_{0}\right)
\end{array}\right)=R\left(\begin{array}{c}
\Lambda_{0}(L) \\
\vdots \\
\Lambda_{\ell-1}(L)
\end{array}\right)
$$

Les égalités (8) et (9) donnent pour finir le théorème 3.1 pour les cônes sous-analytiques (la régularité des matrices $\hat{Q}$ et $R$ est établie en appendice par un calcul explicite pour $X$ un cône polyédral).

\subsection{Le cas général}

Dans cette section $X_{0}$ est un germe d'ensemble sous-analytique fermé, contenant l'origine. Contrairement à la section précédente, on ne suppose plus ici que $X_{0}$ est un cône. On montre le théorème 3.1 en toute généralité, en déformant $X_{0}$ sur son cône tangent. On se donne $\left(X^{j}\right)$ une stratification de Whitney de $X$. Soit $X$ un représentant de $X_{0}$, notons $X_{r}$ le 
sous-analytique compact $\frac{1}{r} \cdot\left(X \cap B_{(0, r)}\right), L_{r}=\frac{1}{r} \cdot\left(X \cap S_{(0, r)}\right), C_{r} X=\mathbb{R}_{+} \cdot L_{r}$. La famille $\left(C_{r} X\right)_{r \in] 0,1]}$ est la déformation de $X_{0}$ sur son cône tangent $C_{0} X$.

Lemme 3.2. - Avec les notations ci-dessus, on a, pour $P$ dans un ouvert sous-analytique dense de $\bar{G}(n-i, n)$ :

$$
\lim _{r \rightarrow 0} \chi\left(X_{r} \cap P\right)=\lim _{r \rightarrow 0} \chi\left(C_{r} X \cap P\right)
$$

Démonstration. - Commençons par prouver ce lemme pour $P$ un hyperplan de $\mathbb{R}^{n}$, i.e. pour $i=1$. Soit donc $P$ un hyperplan affine de $\bar{G}(n-1, n)$ ne passant pas par 0 , et $E$ le demiespace ne contenant pas 0 qu'il définit. L'application $R: E \rightarrow S_{(0,1)}^{n-1}$, définie par $R(x)=$ $x /\|x\|$ est un homéomorphisme de $P \cap B_{(0,1)}$ sur $S_{(0,1)}^{n-1} \cap E$ qui envoie $C_{r} X \cap P$ sur $L_{r} \cap E$. Il suffit alors de prouver que $\lim _{r \rightarrow 0} \chi\left(X_{r} \cap P\right)=\lim _{r \rightarrow 0} \chi\left(L_{r} \cap E\right)$. Soit $x \in P \cap S_{(0,1)}^{n-1}$. Nous appelons angle entre deux sous-espaces $G$ et $H$ de $\mathbb{R}^{n}$ le maximun des angles des droites de $G$ et de $H$. Il s'agit d'une quantité comprise entre 0 et $\frac{\pi}{2}$ que nous notons $\angle(G, H)$. L'angle entre $P$ et $T_{x} S_{(0,1)}^{n-1}$, est plus petit que $\frac{\pi}{2}-C$, pour $C>0$ une certaine constante, puisque $0 \notin P$. On peut déformer $F_{0}=S_{(0,1)}^{n-1} \cap E$ sur $F_{1}=P \cap B_{(0,1)}$ dans une famille telle que l'angle entre $T_{a} F_{t}$ et $T_{R(a)} F_{0}$ soit majoré par $\frac{\pi}{2}-C$, quel que soit $a \in E \cap B_{(0,1)}$. On note $X_{r}^{j}$ la strate de $X_{r}$ provenant de $X^{j}$ et on suppose que $X^{j}$ n'est pas de dimension $n$. Soit alors $r$ suffisamment petit pour que $: \forall x \in X_{r}, \exists \ell_{x} C_{0} X \cap S_{(0,1)}^{n-1}, \angle\left(\ell_{x}, T_{R(x)} F_{0}\right) \geq \frac{\pi}{2}-C / 3$ et $\angle\left(\ell_{x}, T_{x} X_{r}^{j}\right) \leq C / 3$ (lorsque $x \in X_{r}^{j}$ ). Quelle que soit la strate $X_{r}^{j}$ et $x \in X_{r}^{j}$, quel que soit $t \in[0,1]$, l'angle $\angle\left(T_{x} F_{t}, T_{x} X_{r}^{j}\right)$ vérifie :

$$
\begin{aligned}
\angle\left(T_{x} F_{t}, T_{x} X_{r}^{j}\right) & \geq \angle\left(T_{R(x)} F_{0}, \ell_{x}\right)-\angle\left(T_{R(x)} F_{0}, T_{x} F_{t}\right)-\angle\left(\ell_{x}, T_{x} X_{r}^{j}\right) \\
& \geq\left(\frac{\pi}{2}-C / 3\right)-\left(\frac{\pi}{2}-C\right)-C / 3=C / 3>0 .
\end{aligned}
$$

De sorte que la déformation $\left(F_{t}\right)_{t \in[0,1]}$ est transverse aux strates de Whitney $\left(X_{r}^{j}\right)$ de $X_{r}$. Le premier lemme d'isotopie de Thom-Mather assure alors que $F_{0} \cap X_{r}$ et $F_{1} \cap X_{r}$ sont homéomorphes. Lorsque $P$ n'est pas un hyperplan, on place $P$ dans $\pi_{V}^{-1}\left(\mathbb{R} \cdot \pi_{V}(P)\right)$, où $V$ est le $i$-plan vectoriel de $\mathbb{R}^{n}$ orthogonal à $P$. Comme $\operatorname{dim}(X) \geq i$, le raisonnement précédent s'applique pour presque toutes les droites $\mathbb{R} \cdot \pi_{V}(P)$ de $V$.

Lemme 3.3. - Avec les notations précédentes et celles du théorème 2.6:

$$
\lim _{r \rightarrow 0} \sigma_{j}\left(C_{r} X\right)=\sigma_{j}\left(X_{0}\right)
$$

Démonstration. - Soient $P$ un $j$ plan vectoriel de $\mathbb{R}^{n}$ qui coupe $B_{(0,1)}$ et $y \in P$, par le lemme 3.2, pour $r$ suffisamment petit on a : $\chi\left(C_{r} X \cap\left(y+P^{\perp}\right)\right)=\chi\left(X_{r} \cap\left(y+P^{\perp}\right)\right)$. Par définition des constantes $\chi_{1}^{P}\left(X_{0}\right), \cdots, \chi_{n_{P}}^{P}\left(X_{0}\right)$ et des domaines $\mathcal{K}_{1}^{P}\left(X_{0}\right), \cdots, \mathcal{K}_{n_{P}}^{P}\left(X_{0}\right)$ (Théorème 2.6), on a :

$$
\lim _{r \rightarrow 0} \Theta_{j}\left(\left\{y \in P \cap B_{(0,1)} ; \chi\left(X_{r} \cap\left(y+P^{\perp}\right)\right)=\chi_{k}^{P}\left(X_{0}\right)\right\}\right)=\Theta_{j}\left(\mathcal{K}_{k}^{P}\left(X_{0}\right)\right) .
$$

Comme les caractéristiques d'Euler-Poincaré de toutes les sections planes de $X_{r}$ et de $C_{r} X$ sont uniformément bornées relativement à $r$, le théorème de convergence dominée et le lemme 3.2 donnent : 


$$
\begin{aligned}
\lim _{r \rightarrow 0} \sigma_{j}\left(C_{r} X\right) & =\lim _{r \rightarrow 0} \int_{P \in G(j, n)} \sum_{k=1}^{n_{P}^{r}} \chi_{k}^{P}\left(C_{r} X\right) \cdot \Theta_{j}\left(\mathcal{K}\left(C_{r} X\right)\right) d \gamma_{j, n}(P) \\
& =\int_{P \in G(j, n)} \sum_{k=1}^{n_{P}} \chi_{k}^{P}\left(X_{0}\right) \cdot \Theta_{j}\left(\mathcal{K}\left(X_{0}\right)\right) d \gamma_{j, n}(P)=\sigma_{j}\left(X_{0}\right) .
\end{aligned}
$$

Preuve du théorème 3.1. - Comme ci-dessus on utilise le fait que les familles $\left(C_{r} X\right)_{r \in[0,1]}$ et $\left(X_{r}\right)_{r \in[0,1]}$ sont des sous-analytiques compacts et donc que les caractéristiques d'Euler-Poincaré de toutes les sections planes de $X_{r}$ et de $C_{r} X$ sont uniformément bornées relativement à $r$ pour appliquer le théorème de convergence dominée. On a ainsi, par le lemme 3.2 :

$$
\begin{aligned}
\Lambda_{i}^{\mathrm{loc}}\left(X_{0}\right) & =\frac{1}{\alpha_{i} \cdot \beta(n, i)} \lim _{r \rightarrow 0} \int_{\bar{P} \in \bar{G}(n-i, n)} \chi\left(X_{r} \cap \bar{P}\right) d \bar{\gamma}_{n-i, n}(\bar{P}) \\
& =\frac{1}{\alpha_{i} \cdot \beta(n, i)} \lim _{r \rightarrow 0} \int_{\bar{P} \in \bar{G}(n-i, n)} \chi\left(C_{r} X \cap \bar{P}\right) d \bar{\gamma}_{n-i, n}(\bar{P})=\lim _{r \rightarrow 0} \Lambda_{i}^{\mathrm{loc}}\left(C_{r} X\right) .
\end{aligned}
$$

Mais le théorème 3.1 étant vrai pour les cônes :

$$
\Lambda_{i}^{\mathrm{loc}}\left(X_{0}\right)=\lim _{r \rightarrow 0} \sum_{j=i}^{n} m_{i}^{j} \cdot \sigma_{j}\left(C_{r} X\right) .
$$

Ce qui donne par le lemme 3.3 l'égalité annoncée du théorème 3.1 :

$$
\Lambda_{i}^{\mathrm{loc}}\left(X_{0}\right)=\lim _{r \rightarrow 0} \sum_{j=i}^{n} m_{i}^{j} \cdot \sigma_{j}\left(C_{r} X\right)=\sum_{j=i}^{n} m_{i}^{j} \cdot \sigma_{j}\left(X_{0}\right) .
$$

\section{Conditions de régularité et invariants locaux}

Lorsque $X$ est un ensemble sous-analytique, les fonctions $\sigma_{j}$ et $\Lambda_{j}^{\text {loc }}$ sont des fonctions Log-analytiques (voir par exemple [51], [12]). Nous nous intéressons ici à la régularité de ces fonctions. Nous prouvons dans cette section la continuité des invariants $\sigma_{j}$ le long de strates d'une stratification de Verdier d'un ensemble sous-analytique fermé. Il s'agit de montrer que le long (de la projection) d'une telle strate, les discriminants des projections sur des $i$-plans ont un bon comportement du point de vue de la densité des domaines qu'ils bordent : l'équisécabilité. Dans le cadre analytique complexe, l'équisécabilité des discriminants le long d'une strate est la constance de leur multiplicité locale, condition que l'on sait être équivalente à la $(w)$, la $\left(b^{*}\right)$, la $(b)$-régularité et à la constance des caractéristiques évanescentes (cf. Section 2.2). Nous montrons au passage (Corollaire 4.5) l'implication $(i) \Rightarrow(i v)$ du théorème 2.10, c'est-à-dire la constance des caractéristiques évanescentes le long de strates de Whitney d'un ensemble analytique complexe ([5] dans le cas des familles analytiques d'hypersurfaces à singularité isolée, c'est-à-dire pour les nombres de Milnor des sections planes). Bien qu'annoncée par ailleurs, il s'agit à notre connaissance de la seule preuve dans le cas général de cette implication qui n'utilise pas le calcul qui lie la multiplicité des variétés polaires aux caractéristiques évanescentes. 
Rappelons que si $E$ est une condition de régularité portant sur un couple $(Y, Z)$ de sousvariétés de $\mathbb{R}^{n}$, dans [78] et [80] est définie la $E_{\operatorname{cod}(\ell)}$-régularité $(0 \leq \ell \leq \operatorname{cod}(Y))$ de la façon suivante : le couple $(Y, Z)$ est dit $E_{\operatorname{cod}(\ell)}$-régulier en $y \in Y$, s'il existe un ouvert dense $\mathcal{M}_{y}$ dans $\left\{\Pi \in G(n-\ell, n), \mathrm{T}_{y} Y \subset \Pi\right\}$ pour lequel on ait, quelle que soit la sous-variété $\mathcal{W}$ de $\mathbb{R}^{n}$ telle que $Y \subset \mathcal{W}$ dans un voisinage de $y$, l'implication : $\mathrm{T}_{y} \mathcal{W} \in \mathcal{M}_{y} \Longrightarrow Z$ et $\mathcal{W}$ sont transverses dans un voisinage de $y$ et $(Y, \mathcal{W} \cap Z)$ est $E$-régulier en $y$. Lorsque $(Y, Z)$ est $E_{\operatorname{cod}(\ell)}$-régulier en $y$ pour tout $\ell, 0 \leq \ell \leq \operatorname{cod}(Y)$, on dit que $(Y, Z)$ est $\left(E^{*}\right)$-régulier en $y$. On se reportera par exemple à [57], [59], [60] et [61] pour une étude comparée de la $\left(r^{*}\right)$, de la $\left(b^{*}\right)$ et de la $\left(w^{*}\right)$-régularité. Notons que la condition $\left(b^{*}\right)$ est strictement plus faible que la condition $(w)$, même en algébrique. En effet, d'après [59], pour la catégorie sous-analytique $(w) \Longrightarrow\left(b^{*}\right)$, puis d'une part d'après [59, Corollaire 3.13], si le couple de strates $(Y, Z)$ est (b)-régulier et si $\operatorname{dim}(Y)=1$, ce couple est aussi $\left(b^{*}\right)$-régulier, et d'autre part dans [7] et [79] sont donnés des exemples de couples $(Y, Z)$ qui sont $(b)$-réguliers mais non $(w)$-réguliers et où $\operatorname{dim}(Y)=1$.

Nous utilisons dans cette partie la condition $(*)$ suivante qui porte sur une projection $\pi_{P}$, avec $P \in G(i, n)$ et la stratification $\left(X^{j}\right)_{j \in\{0, \cdots, k\}}$ de $X$ que l'on se donne :

Condition. - La projection $\pi_{P}: \mathbb{R}^{n} \rightarrow P$ est une projection pour laquelle existe un voisinage ouvert $\mathcal{U}\left(=\mathcal{U}_{P}\right)$ de l'origine tel que quelle que soit la strate $X^{j}$ adhérente à $X^{0}$ :

$$
\mathcal{U} \cap \mathcal{P}_{X^{j}}(P) \cap\left(\pi_{P}^{-1}\left(\pi_{P}\left(X^{0}\right)\right) \backslash X^{0}\right)=\varnothing
$$

En nous appuyant sur le théorème 4.10, nous remarquons dans la proposition qui suit que la condition $(*)$ est une condition qui a lieu pour des projections $\pi_{P}$ génériques, dès lors que la stratification $\left(X^{j}\right)_{j \in\{0, \cdots, k\}}$ est $(w)$-régulière.

Proposition 4.1. - Soit X un ensemble sous-analytique fermé de $\mathbb{R}^{n}$, contenant l'origine, muni d'une stratification $(w)$-régulière $\left(X^{j}\right)_{j \in\{0, \cdots, k\}}$ et soit $X^{0}$ la strate contenant 0 . Soit $i$ un entier. Il existe un ensemble sous-analytique dense $\mathcal{G}_{X}^{i}$ dans $G(i, n)$, tel que pour tout $P \in \mathcal{G}_{X}^{i}$, $\pi_{P}: \mathbb{R}^{n} \rightarrow P \in G(i, n)$ est une projection pour laquelle existe un voisinage ouvert $\mathcal{U}\left(=\mathcal{U}_{P}\right)$ de l'origine tel que quelle que soit la strate $X^{j}$ adhérente à $X^{0}$ :

$$
\mathcal{U} \cap \mathcal{P}_{X^{j}}(P) \cap\left(\pi_{P}^{-1}\left(\pi_{P}\left(X^{0}\right)\right) \backslash X^{0}\right)=\varnothing .
$$

Démonstration. - Il s'agit d’une conséquence immédiate du théorème 4.10.(iii).

REMARQUe. - Dans la proposition 4.1, on peut se contenter de supposer que la stratification $\left(X^{j}\right)_{j \in\{0, \cdots, k\}}$ est seulement $\left(a^{*}\right)$-régulière, condition ad hoc pour que $(*)$ ait génériquement lieu. En effet, si $P \in G(i, n)$ est tel que la propriété $(*)$ n'a pas lieu, il existe une strate $X^{j}$ contenant dans son adhérence $X^{0}$, une suite $\left(x_{n}\right)_{n \in \mathbb{N}}$ de limite 0 et contenue dans $P_{X^{j}}(P) \cap\left(\pi_{P}^{-1}\left(X^{0}\right) \backslash X^{0}\right)$. Soit $q=\operatorname{dim}\left(X^{j}\right)$. En chaque point $x_{n}, P^{\perp}$ et $\mathrm{T}_{x_{n}} X^{j}$ ont une intersection excédentaire en dimension $\max (0, q-i+1)$. Supposons $i \in\left[\operatorname{dim}\left(X^{0}\right), \operatorname{dim}(X)\right]$, qui est le seul cas intéressant. Supposons que dans un voisinage de 0 la strate $X^{0}$ soit un sousespace vectoriel de $\mathbb{R}^{n}$ de dimension $d$. Si $P$ est générique, $X^{0} \cap P^{\perp}=\{0\}$ et si la stratification $\left(X^{j}\right)_{0 \in\{0, \cdots, k\}}$ est $\left(a^{*}\right)$-régulière, par définition même il existe un sous-analytique dense 
$\mathcal{W}_{n-i+d}$ de l'ensemble des $(d+n-i)$-plans contenant $X^{0}$ tel que si $X^{0} \oplus P^{\perp}=W \in \mathcal{W}_{n-i+d}$, alors $W$ vérifie :

$$
\pi_{P}^{-1}\left(\pi_{P}\left(X^{0}\right)\right)=W \text { coupe transversalement } X^{j}
$$

et

$$
\left(X^{0}, X^{j} \cap W\right) \text { est un couple de strates }(a) \text {-régulier en } 0 .
$$

On a, d'après (2) : $\operatorname{dim}\left(W \cap X^{j}\right)=q-i+d$ et d'après ce qui précède, $P^{\perp}$ et $\mathrm{T}_{x_{n}}\left(X^{j} \cap\right.$ $W$ ) ont une intersection en dimension au moins $q-i+1$. Comme $P^{\perp}$ est transverse à $X^{0}$, $\lim _{n \rightarrow \infty} \mathrm{T}_{x_{n}}\left(X^{j} \cap W\right)$ ne peut pas contenir l'espace $X^{0}$ qui est de dimension $d$, ce qui contredit (3).

Comme la $\left(b^{*}\right)$-régularité, la $\left(a^{*}\right)$-régularité est une condition strictement plus faible que la $(w)$-régularité et on ne sait pas, par exemple, si en sous-analytique $(b) \Longrightarrow\left(a^{*}\right)$, comme c'est le cas en complexe.

Rappelons maintenant que les caractéristiques $\chi_{j}^{P}(0)$ sont définies de la façon suivante (cf. Théorème 2.6.(iii) pour les notations) : si $X$ est un ensemble sous-analytique fermé de $\mathbb{R}^{n}$ contenant 0 et si $P$ est un $i$-plan vectoriel (général) de $\mathbb{R}^{n}$, il existe un réel non nul $R_{0}=$ $R_{0}(P)$, tel que pour tout $r \leq R_{0}$, les ensembles sous-analytiques ouverts $K_{1}^{P, r}, \cdots, K_{N_{P}}^{P, r}$, qui sont les composantes connexes du complémentaire dans $P$ de $\mathcal{D}_{L_{r}}(P) \cup \bigcup_{j=0}^{k} \mathcal{D}_{X^{j} \cap B_{(0, r)}^{n}}(P)$, ont une réunion dense dans $P$ au voisinage de 0 , et vérifient :

- les germes $\left(K_{j}^{P, r}\right)_{0}$ et $\left(K_{j}^{P, s}\right)_{0}$ coïncident pour $r, s \leq R_{0}$,

- pour tout $y$ suffisamment proche de $\pi_{P}(0)=0$ dans un domaine $K_{j}^{P, r}$, (i.e. $0<\|y\| \ll r$ ), l'entier $\chi_{j}^{P}=\chi\left(\pi_{P}^{-1}(y) \cap X \cap B_{(0, r)}^{n}\right)$ ne dépend pas de $r$ (lorsque $r \leq R_{0}$ ).

Notation 4.2. - On notera $\mathcal{R}_{0}=\mathcal{R}_{0}(P)$ le plus grand rayon $R_{0}$ qui vérifie cette proposition.

Remarque. - D'après les propositions 2.3, 2.4 et le lemme d'isotopie de Thom-Mather, l'existence du rayon $R_{0}(P)$ est garantie dès que $P \in \mathcal{E}_{X}^{i}$ (notation qui précède la proposition 2.3), dès que pour tout $j \in\{0, \cdots, k\}, P^{\perp}$ ne rencontre pas $\mathcal{P}_{X^{j}}(P)$ dans $B\left(0, R_{0}(P)\right)$ et lorsque pour tout $r \leq R_{0}(P), S(0, r)$ est transverse à toutes les strates $X^{j}$ et $0 \notin \mathcal{D}_{L_{r}}(P)$. De sorte que si $R>\mathcal{R}_{0}$, pour tout $\eta>0$, il existe $y_{\eta} \in P, j \in\{0, \cdots, k\}$ et $x_{\eta} \in X$ tels que :

- $0<\left\|y_{\eta}\right\| \leq \eta, \mathcal{R}_{0} \leq \lim _{\eta \rightarrow 0}\left\|x_{\eta}\right\| \leq R, x_{\eta} \in X^{j} \cap \pi_{P}^{-1}\left(y_{\eta}\right)$,

- $\pi_{P}^{-1}\left(y_{\eta}\right) \cap T_{x_{\eta}} X^{j}$ ne soit pas transverse en $x_{\eta}$ à $S_{\left(0,\left\|x_{\eta}\right\|\right)}$.

Nous montrons maintenant dans la proposition 4.3 que la proposition 2.5, qui est locale en un point, admet une version le long d'une strate d'une stratification suffisamment régulière. En particulier : si $Y$ est une strate d'une stratification $\left(b^{*}\right)$-régulière de $X$, si $P$ est générique dans $G(i, n)$, il existe un voisinage $\mathcal{U}$ de 0 dans $Y$, deux réels $0<r \ll R$, tels que pour tout $y \in Y \cap \mathcal{U}$, pour tout $z$ et $z^{\prime}$ dans le même profil polaire $\mathrm{K}_{j}^{P, R}(y)$ avec $\|z-y\|<r$ et $\left\|z^{\prime}-y\right\|<r, \pi_{P}^{-1}(z) \cap X \cap B(y, R)$ et $\pi_{P}^{-1}\left(z^{\prime}\right) \cap X \cap B(y, R)$ sont homéomorphes. 
Proposition 4.3. - Soit $X$ un ensemble sous-analytique fermé de $\mathbb{R}^{n}$ muni d'une stratification (b)-régulière $\left(X^{j}\right)_{j \in\{0, \cdots, k\}}$. Soient $X^{0}$ la strate contenant $0, i \in\{0, \ldots, n\}$ et $\pi_{P}$ : $\mathbb{R}^{n} \rightarrow P \in G(i, n)$ une projection telle qu'existe un voisinage ouvert $\mathcal{U}$ de l'origine pour lequel, quelle que soit la strate $X^{j}$ adhérente à $X^{0}$ :

$$
\mathcal{U} \cap \mathcal{P}_{X^{j}}(P) \cap\left(\pi_{P}^{-1}\left(\pi_{P}\left(X^{0}\right)\right) \backslash X^{0}\right)=\varnothing .
$$

Si de plus les intersections $\pi_{P}^{-1}\left(X^{0}\right) \cap X^{j}$ sont (b)-régulières le long de $X^{0}$, on a :

(i) Le rayon $r_{P}^{\prime}(y)$ de la proposition 2.5 est minoré par un réel $R>0$, indépendant de $y \in X^{0}$ (dans un voisinage de 0 ).

(ii) Quel que soit $j \in\{0, \cdots, k\}$, la distance $\delta(y)$ de $\mathcal{D}_{L_{X^{j}}^{R}(y)}$ à y ne tend pas vers 0 lorsque $y$ tend vers 0 dans $X^{0}$.

Démonstration. - (i) Notons que pour chaque $y \in X^{0}, P \in \mathcal{E}_{X}^{i}(y)$, par la condition $(*)$. Soit $y \in X^{0}$. La preuve de la proposition 2.5 (cf. la remarque qui la suit) montre que $r_{P}^{\prime}(y)$ est réalisé dès que :

- pour $r \leq r_{P}^{\prime}(y)$, les sphères $S(y, r)$ sont transverses aux strates $X^{j}$,

- en tout point $x$ de $B\left(y, r_{P}^{\prime}\right), \mathrm{T}_{x}\left(X^{j} \cap\left(y+P^{\perp}\right)\right)$ et la direction $y-x$ ne sont pas orthogonaux.

Pour le premier point : on peut choisir un réel $R>0$ indépendant de $y$ tel que pour tout $y \in X^{0}, S(y, r)$ et $X^{j}$ sont transverses pour $r \leq R$. Ceci résulte de la condition $(b)$. Le second point résulte de la condition (b) pour le couple $\left(X^{0}, \pi_{P}^{-1}\left(X^{0}\right) \cap X^{j}\right)$.

(ii) On choisit $R$ comme dans (i) et on regarde les links $L_{X^{j}}^{R}(y)$ des strates $X^{j}$ en les points $y$ de $X^{0}$. D'après (i), la distance de leur discriminant à $y$, notée $\delta(y)$ n'est pas nulle. On veut montrer que $\delta(y)$ est minorée par $\delta>0$ indépendamment de $y$. Autrement dit qu'il y existe un voisinage de 0 dans $P$ dans lequel n'entrent pas les discrimants de la famille de links $\left(L_{X^{j}}^{R}(y)\right)_{y \in X^{0}, j \in\{0, \cdots, k\}}$. Si tel n'est pas le cas, on trouve une suite de points $\left(x_{\ell}^{R}\right)_{\ell \in \mathbb{N}}$ avec $x_{\ell}^{R} \in S\left(y_{\ell}, R\right), y_{\ell} \in X_{0}$ telle que $\mathrm{T}_{x_{\ell}^{R}}\left[L_{X^{j}}^{R}\left(y_{\ell}\right)\right]=\mathrm{T}_{x_{\ell}^{R}} S_{\left(y_{\ell}, R\right)} \cap \mathrm{T}_{x_{\ell}^{R}} X^{j}$ et $P^{\perp}$ soient non transverses et $\lim _{\ell \rightarrow \infty} \pi_{P}\left(x_{\ell}^{R}\right)=0$. Comme on l'a remarqué dans la preuve de la proposition 2.5, pour $\pi_{P}\left(x_{\ell}^{R}\right)$ suffisamment proche de $0, \pi_{P}\left(x_{\ell}^{R}\right)+P^{\perp}$ et $X^{j}$ sont transverses en $x_{\ell}^{R}$ (par l'hypothèse $(*)$ et la condition $(a)$ ) et de ce fait on obtient l'inclusion : $\mathrm{T}_{x_{\ell}^{R}} X^{j} \cap P^{\perp} \subset$ $\mathrm{T}_{x_{\ell}^{R}} S\left(y_{\ell}, R\right)$. En faisant $\ell \rightarrow \infty$, on en déduit un point $x^{R} \in S(0, R) \cap X^{m}$, pour une certaine strate $X^{m}$ et par la condition $(a)$ au point $x^{R}$ pour le couple $\left(X^{m}, X^{j}\right)$, l'inclusion $\mathrm{T}_{x_{\ell}^{R}} X^{j} \cap P^{\perp} \subset \mathrm{T}_{x_{\ell}^{R}} S\left(y_{\ell}, R\right)$ donne par passage à la limite : $\mathrm{T}_{x^{R}} X^{m} \cap P^{\perp} \subset \mathrm{T}_{x^{R}} S(0, R)$, ce qui contredit $\delta(0) \neq 0$.

Proposition 4.4. - Soit $X$ un ensemble sous-analytique fermé de $\mathbb{R}^{n}$, contenant l'origine, muni d'une stratification $\left(b^{*}\right)$-régulière $\left(X^{j}\right)_{j \in\{0, \cdots, k\}}$ et soit $X^{0}$ la strate contenant 0 . Soit $\pi_{P}: \mathbb{R}^{n} \rightarrow P \in G(i, n)$ une projection telle qu'existe un voisinage ouvert $\mathcal{U}$ de l'origine pour lequel quelle que soit la strate $X^{j}$ adhérente à $X^{0}$ :

$$
\mathcal{U} \cap \mathcal{P}_{X^{j}}(P) \cap\left(\pi_{P}^{-1}\left(\pi_{P}\left(X^{0}\right)\right) \backslash X^{0}\right)=\varnothing .
$$

(i) La projection $\pi_{P}$ permet de définir les caractéristiques $\chi_{j}^{P}(y)$ (à la façon du théorème 2.6.(iii)), quel que soit $y \in X^{0} \cap \mathcal{U}$. 
(ii) Il existe un ensemble sous-analytique $\mathcal{L}_{X}^{i}$ dense dans $G(i, n)$ tel que pour $P \in \mathcal{L}_{X}^{i}$ existent un voisinage ouvert $\mathcal{U}^{\prime}=\mathcal{U}^{\prime}(P)$ de 0 dans $\mathbb{R}^{n}$ et un réel $\mathcal{R}=\mathcal{R}(P)>0$, tels que pour tout $j \in\{0, \cdots, k\}$ :

- $\bigcup_{y \in \mathcal{U}^{\prime}} B(y, \mathcal{R}) \cap \mathcal{P}_{X^{j}}(P) \cap\left(\pi_{P}^{-1}\left(\pi_{P}\left(X^{0}\right)\right) \backslash X^{0}\right)=\varnothing$,

- pour tout $y \in X^{0} \cap \mathcal{U}^{\prime}, \mathcal{R} \leq \mathcal{R}_{y}(P)$.

En conséquence chaque caractéristique $\chi_{\ell}^{P}(y)$ en y est donnée par $\chi\left(\pi_{P}^{-1}(z) \cap X \cap B(y, \mathcal{R})\right)$, pour $z$ suffisamment proche de $y$ dans $P$.

Pour tout $P$ dans le sous-analytique $\mathcal{L}_{X}^{i} \subset G(i, n)$, pour le réel $\mathcal{R}=\mathcal{R}(P)$ donné en (ii) et avec les notations de la définition 2.1 et du théorème 2.6, il existe un voisinage de 0 dans $X^{0}$, tel que pour tout y dans ce voisinage:

(iii) L'ensemble $\bigcup_{j=0}^{k} \mathcal{D}_{X^{j} \cap B_{(0, \mathcal{R})}}(P)$ est un représentant du discriminant local $\Delta_{X_{y}}(P)$ de $X$ en y et chaque profil polaire local $\mathcal{K}_{\ell}^{P}(y)$ de $X$ en y admet pour représentant un domaine choisi parmi les $K_{1}^{P, \mathcal{R}}(0), \cdots, K_{N_{P}}^{P, \mathcal{R}}(0)$ qui contiennent 0 dans leur adhérence.

(iv) Chaque entier $\chi_{\ell}^{P}(y)$ associé au profil polaire local $\mathcal{K}_{\ell}^{P}(y)$ s'obtient comme $\chi\left(\pi_{P}^{-1}(z) \cap\right.$ $\left.X \cap B_{(0, \mathcal{R})}^{n}\right)$, pour $z$ suffisamment proche de y dans le domaine $K_{m_{\ell}}^{P, \mathcal{R}}(0)$ qui représente $\mathcal{K}_{\ell}^{P}(y)$, et en particulier $\chi_{\ell}^{P}(y)=\chi_{m_{\ell}}^{P}(0)$.

Démonstration. - (i) La stratification $\left(X^{j}\right)_{j \in\{0, \cdots, k\}}$ étant une stratification ( $\left.b^{*}\right)$ régulière, il s'agit aussi d'une stratification $(b)$-régulière. D'après les hypothèses, la projection $\pi_{P}$ est dans l'ouvert $\mathcal{E}_{X}^{i}=\mathcal{E}_{X}^{i}(y)$ défini dans la section 2 et attaché à chaque point $y$ de $X^{0} \cap \mathcal{U}$. On peut alors appliquer le théorème $2.6 ; \pi_{P}$ est bien une projection qui permet de définir les profils polaires locaux $\mathcal{K}_{j}^{P}$ et les caractéristiques $\chi_{j}^{P}$ qui leur correspondent.

(ii) La stratification $\left(X^{j}\right)_{j \in\{0, \cdots, k\}}$ étant par hypothèse une stratification $\left(b^{*}\right)$-régulière, en particulier l'ensemble des $P \in G(i, n)$ vérifiant la condition (*) est un sous-analytique dense dans $G(i, n)$ (cf. la remarque qui suit la proposition 4.1). Si de plus $P$ est choisi de sorte que les couples $\left(X^{0}, \pi_{P}^{-1}\left(X^{0}\right) \cap X^{j}\right)$ soient (b)-réguliers, d'après la proposition 4.3.(i) l'existence de $\mathcal{U}^{\prime}$ et de $\mathcal{R}$ est garantie. Or un tel choix donne encore un ensemble sous-analytique dense de $G(i, n)$, puisque la stratification $\left(X^{j}\right)_{j \in\{0, \cdots, k\}}$ est $\left(b^{*}\right)$-régulière.

(iii) Pour tout $y$ dans un voisinage de 0 dans $X^{0},\left(B(0, \mathcal{R}) \cap \pi_{P}^{-1}(y) \cap \mathcal{P}_{X^{j}}\right) \backslash\{y\}=\varnothing$, puisque $P \in \mathcal{L}_{X}^{i}$. La remarque qui précède la preuve de la proposition 2.3 montre que cette condition suffit pour que $\Delta_{X_{y}^{j}}(P)=\left(\mathcal{D}_{X^{j} \cap B_{(y, \mathcal{R})}}(P)\right)_{y}=\left(\mathcal{D}_{X^{j} \cap B_{(0, \mathcal{R})}}(P)\right)_{y}$. On remarque ensuite que $\Delta_{X_{y}}(P)$ est le bord de la réunion des profils polaires locaux $\mathcal{K}_{\ell}^{P}(y)$.

(iv) Soit $\delta>0$ un réel minorant la distance $\delta(y)$ de $\mathcal{D}_{L_{X^{j}}^{\mathcal{R}}}(y)$ à $y$ (Proposition 4.3.(ii)), pour $j \in\{0, \cdots, k\}$ et pour tout $y$ voisin de 0 dans $X^{0}$. Soit $y$ proche de 0 dans $X^{0}$ (et $\|y\| \ll \min (\delta, \mathcal{R}))$ de sorte que les profils polaires locaux de $y$ admettent pour représentant certains des domaines $K_{1}^{P, \mathcal{R}}(0), \cdots, K_{N_{P}}^{P, \mathcal{R}}(0)$ (d'après le point (iii)) et soit $z \in P$ tel que $\|y-z\| \ll \delta$. Alors d'une part, pour un certain $\ell, \chi\left(\pi_{P}^{-1}(z) \cap B(y, \mathcal{R}) \cap X\right)=\chi_{\ell}^{P}(y)$ et le profil polaire local $\mathcal{K}_{\ell}^{P}(y)$ admet le domaine $K_{m \ell}^{P, \mathcal{R}}(0)$ pour représentant, et d'autre part $\chi\left(\pi_{P}^{-1}(z) \cap B(y, \mathcal{R}) \cap X\right)=\chi\left(\pi_{P}^{-1}(z) \cap B(0, \mathcal{R}) \cap X\right)$, puisque $\|y\| \ll \delta$ et $\|y-z\| \ll \delta$. Enfin $\chi\left(\pi_{P}^{-1}(z) \cap B(0, \mathcal{R}) \cap X\right)=\chi_{m_{\ell}}^{P}(0)$.

On en vient maintenant à l'implication (i) $\Rightarrow$ (iv) du théorème 2.10 que l'on s'est proposé de démontrer. 
Corollaire 4.5. - Soit $X$ un ensemble analytique complexe de $\mathbb{C}^{n}$ muni d'une stratifcation analytique complexe (b)-régulière $\left(X^{j}\right)_{j \in\{0, \cdots, k\}}$. La suite des caractéristiques évanescentes $\left(\widetilde{\sigma}_{i}\left(X_{y}\right)\right)_{i \in\{1, \cdots, n\}}$ est constante lorsque y varie le long des strates de $\left(X^{j}\right)_{j \in\{0, \cdots, k\}}$.

Démonstration. - Voyons $X$ comme un sous-analytique de $\mathbb{R}^{2 n}$ et montrons la constance des caractéristiques évanescentes le long de la strate $X^{0}$ au voisinage de $0 \in X^{0}$. Notons que la stratification $\left(X^{j}\right)_{j \in\{0, \cdots, k\}}$ du sous-analytique $X$ est $\left(b^{*}\right)$-régulière, puisque (b)-régulière et analytique complexe. Soit $i \in\{1, \cdots, n\}$. On remarque que l'ensemble $\mathcal{L}_{X}^{i}$ de la proposition 4.4.(ii) peut être choisi dense dans $\widetilde{G}(i, n)$ aussi bien que dans $G(i, n)$, avec les mêmes arguments. Dans ces conditions, soit $P \in \mathcal{L}_{X}^{i}$ et $\mathcal{K}^{P}(0)$ l'unique profil polaire local de $X$ en 0 (puisque le complémentaire de $\Delta_{X_{0}}(P)$ est connexe). La proposition 4.4.(iv) montre que si $y$ est dans un voisinage de 0 dans $X^{0}, \mathcal{K}^{P}(y)$ et $\mathcal{K}^{P}(0)$ admettent un même représentant $K^{P, r}(0)$ et que : $\widetilde{\sigma}_{i}\left(X_{0}\right)=\widetilde{\sigma}_{i}\left(X_{y}\right)$.

Remarque. - L'implication utile dans la preuve du corollaire 4.5 est $(b) \Longrightarrow\left(b^{*}\right)$. Implication dont on répète qu'on ne sait pas si elle vraie en sous-analytique réel.

Pour $X$ un sous-ensemble de $\mathbb{R}^{n}$ et $Y=\mathbb{R}^{d} \times\{0\}^{n-d}$, on note $\mathcal{D}(X, Y)$ la déformation de $X$ sur son cône normal à $Y$. Il s'agit du sous-ensemble suivant de $\mathbb{R}^{n} \times \mathbb{R}$ :

$$
\mathcal{D}(X, Y)=\operatorname{adh}\left\{(y, \ell, u) \in \mathbb{R}^{d} \times \mathbb{R}^{n-d} \times \mathbb{R}_{+}^{*} ;(y, u \ell) \in X \backslash Y\right\} .
$$

L'application $u: \mathcal{D}(X, Y) \longrightarrow \mathbb{R}$ induite par la deuxième projection est appelée déformation de $X$ au cône normal à $Y$ dans $X$. Cette application est compatible avec l'action de $\mathbb{R}_{+}^{*}$ définie $\operatorname{par}(\lambda,(y, \ell, u)) \longmapsto\left(y, \lambda \ell, \lambda^{-1} u\right)$ pour $\lambda$ dans $\mathbb{R}_{+}^{*}$. Sa fibre en $0 \in \mathbb{R}$ est le cône normal à $Y$ dans $X$, noté $C_{Y} X$ et stable par l'action induite de $\mathbb{R}_{+}^{*}$ décrite $\operatorname{par}(\lambda,(y, \ell)) \longmapsto(y, \lambda \ell)$ pour $\lambda$ dans $\mathbb{R}_{+}^{*}$. Si $Y=\{0\}, C_{Y} X$ est le cône tangent à $X$ en 0 . La première projection de $\mathbb{R}^{n} \times \mathbb{R}$ induit une application $p: C_{Y} X \longrightarrow Y$. Nous notons $\left(C_{Y} X\right)_{y}$ la fibre $p^{-1}(y)$. Une telle fibre est non vide si et seulement si $y \in \operatorname{adh}(X \backslash Y)$. L'application $(y, \ell, u) \longmapsto(y, u \ell, u)$ induit un isomorphisme de $\mathcal{D}(X, Y) \backslash u^{-1}(0) \operatorname{sur} \operatorname{adh}(X \backslash Y) \times \mathbb{R}_{+}^{*}$.

DÉfinition 4.6. - On dit que $X$ est normalement pseudo-plat le long de $Y$ si la projection $p: C_{Y} X \rightarrow Y$ est ouverte (cf. [34]).

La dimension des fibres de $p$, la pseudo-platitude normale, la condition (b) de Whitney et l'équimultiplicité de $X$ le long de $Y$ lorsque $X$ et $Y$ sont analytiques complexes sont liés par la proposition suivante :

Proposition 4.7. - Soit $X$ et $Y$ deux ensembles sous-analytiques de $\mathbb{R}^{n}$, $X$ étant de dimension d et $Y$ lisse de dimension $k$.

(i) Si $Y$ est une strate d'une stratification de Whitney de $\operatorname{adh}(X), \operatorname{adh}(X)$ est normalement pseudo-plat le long de $Y$, et si $y \in Y, C_{\{y\}}\left(X \cap\left(y+Y^{\perp}\right)\right)=\left(C_{Y} X\right)_{y}$. En particulier le long de strates de Whitney: $\operatorname{dim}\left(\left(C_{Y} X\right)_{y}\right) \leq d-k$ (cf. [34], [32], [61]).

(ii) Si $X$ est normalement pseudo-plat le long de $Y$, quel que soit $y \in Y, \operatorname{dim}\left(\left(C_{Y} X\right)_{y}\right) \leq$ $d-k$ (cf. [34], [32], [61], [11, Lemme 2.4]). 
(iii) Soit $y \in Y$. Notons $s=\operatorname{dim}\left(\left(C_{Y} X\right)_{y}\right)$. Il existe un ensemble sous-analytique $\mathcal{Q}_{y}^{q}$ dense dans $G(q, n)$, tel que pour tout $P \in \mathcal{Q}_{y}^{q}$ on ait l'existence d'un voisinage $\mathcal{U}_{y, P}$ de $y$ dans $\mathbb{R}^{n}$ pour lequel :

$$
\left(\operatorname{adh}\left(X \cap \mathcal{U}_{y, P}\right) \backslash Y\right) \cap \pi_{P}^{-1}\left(\pi_{P}(Y)\right)=\varnothing
$$

si et seulement si $s+k \leq q\left(c f\right.$. [11, Lemme 2.3]). Lorsque $\mathcal{Q}_{y}^{d}$ existe, on dit que $X$ est équisécable le long de $Y$ en $y$; cette condition équivaut à $\operatorname{dim}\left(\left(C_{Y} X\right)_{y}\right) \leq d-k$ (cf. [32]).

(iv) Si $X$ et $Y$ sont des ensembles analytiques complexes de $\mathbb{C}^{n}$, la pseudo-platitude normale de $X$ le long de $Y$, l'équimultiplicité de $X$ le long de $Y$, l'équisécabilité de $X$ le long de $Y$ et la condition $\operatorname{dim}\left(\left(C_{Y} X\right)_{y}\right)=d-k$ pour tout $y \in Y$, sont équivalentes (cf. [34], [65], [32]).

Dans [11, Proposition 2.6], il est montré :

Proposition 4.8. - Soient $\mathcal{K}$ et $Y$ deux ensembles sous-analytiques de $\mathbb{R}^{i}, \mathcal{K}$ étant de dimension $i$ et $Y$ lisse. Notons $\operatorname{int}(\mathcal{K})$ l'intérieur de $\mathcal{K}$ et $\operatorname{tr}(\mathcal{K})=\operatorname{adh}(\mathcal{K}) \backslash \operatorname{int}(\mathcal{K})$ la frontière de $\mathcal{K}$. Si pour tout $y \in Y, \operatorname{dim}\left(\left(C_{Y} \operatorname{fr}(\mathcal{K})\right)_{y}\right) \leq i-1-\operatorname{dim}(Y)$, c'est-à-dire si $\operatorname{fr}(\mathcal{K})$ est équisécable le long de $Y$, la fonction : $Y \ni y \mapsto \Theta_{i}\left(\mathcal{K}_{y}\right)$ est continue.

Dans le théorème qui suit, on identifie une fois de plus une strate $Y$ de $\left(X^{j}\right)_{\{0, \cdots, k\}}$ et son projeté sur un plan vectoriel. Ce théorème montre que l'équisécabilité (ou de façon équivalente, d'après la proposition 4.9.(iii), la majoration adéquate de la dimension des cônes normaux le long de $Y$ ) des discriminants généraux en toute dimension le long des strates d'une stratification $\left(b^{*}\right)$-régulière implique la continuité des invariants $\sigma_{*}$ et $\Lambda_{*}^{\text {loc }}$ le long des strates.

ThÉorème 4.9. - Soit $X$ un ensemble sous-analytique fermé de $\mathbb{R}^{n}$ de dimension $d$, muni d'une stratification $\left(b^{*}\right)$-régulière $\left(X^{j}\right)_{j \in\{0, \cdots, k\}}$ dont $Y$ soit une strate. Soient $i \in\{\operatorname{dim}(Y)+$ $1, \cdots, d\}$ et $\mathcal{L}_{X}^{i}$ le sous-analytique dense de $G(i, n)$ de la proposition 4.4.(ii) (relatif à $Y$ ).

(i) Si pour un ensemble sous-analytique dense $\mathcal{D}^{i} \subset \mathcal{L}_{X}^{i}$ de $G(i, n)$, on a :

$$
P \in \mathcal{D}^{i} \Longrightarrow \operatorname{dim}\left(\left(C_{Y} \Delta_{X_{y}}(P)\right)_{y}\right) \leq i-1-\operatorname{dim}(Y), \forall y \in Y,
$$

alors la restriction à $Y$ de l'invariant $\sigma_{i}\left(X_{y}\right)$ est continue en tout point de $Y$.

(ii) Si le sous-analytique dense $\mathcal{D}^{i}$ existe pour tout $i \in\{\operatorname{dim}(Y)+1, \cdots, d\}$, les restrictions à $Y$ des invariants $\sigma_{1}\left(X_{y}\right), \cdots, \sigma_{n}\left(X_{y}\right)$ et $\Lambda_{1}^{\text {loc }}\left(X_{y}\right), \cdots, \Lambda_{n}^{\text {loc }}\left(X_{y}\right)$ sont continues en tout point de $Y$.

Démonstration. - D'après le théorème 3.1 , le point (i) entraine le point (ii). Montrons alors (i). Soient $y \in Y$ et $\left(y_{n}\right)_{n \in \mathbb{N}}$ une suite de $Y$ de limite $y$. Comme la famille $\left(\chi_{j}^{P}\right)_{P \in \mathcal{E}_{X}^{i}, j \in\left\{1, \cdots, n_{P}\right\}}$ est uniformément bornée, il suffit de prouver que pour $P$ général dans $\mathcal{L}_{X}^{i}$ a lieu l'égalité :

$$
\lim _{n \rightarrow \infty} \sum_{j=1}^{n_{P}\left(y_{n}\right)} \chi_{j}^{P}\left(y_{n}\right) \cdot \Theta_{i}\left(\mathcal{K}_{j}^{P}\left(y_{n}\right)\right)=\sum_{j=1}^{n_{P}(y)} \chi_{j}^{P}(y) \cdot \Theta_{i}\left(\mathcal{K}_{j}^{P}(y)\right) .
$$

Soit alors $P \in \mathcal{L}_{X}^{i} \cap \mathcal{D}^{i}$. D'après la proposition 4.4.(iii) et (iv), il existe un voisinage de $y$ dans $Y$, tel que pour tout $y_{n}$ dans ce voisinage, pour tout $j \in\left\{1, \cdots, n_{P}\left(y_{n}\right)\right\}$, il existe 
$\ell_{j} \in\left\{1, \cdots, n_{P}(y)\right\}$ tel que $\chi_{j}^{P}\left(y_{n}\right)=\chi_{\ell_{j}}^{P}(y)$ et $\mathcal{K}_{j}^{P}\left(y_{n}\right)=K_{\ell_{j}}^{P, r}(y)$. Avec la convention $\Theta_{i}\left(K_{j}^{P, r}(y), y_{n}\right)=0$ si $y_{n} \notin \operatorname{adh}\left(K_{j}^{P, r}(y)\right)$, on obtient pour $n$ suffisamment grand :

$$
\sum_{j=1}^{n_{P}\left(y_{n}\right)} \chi_{j}^{P}\left(y_{n}\right) \cdot \Theta_{i}\left(\mathcal{K}_{j}^{P}\left(y_{n}\right)\right)=\sum_{j=1}^{n_{P}(y)} \chi_{j}^{P}(y) \cdot \Theta_{i}\left(K_{j}^{P, r}(y), y_{n}\right) .
$$

Or puisque $\operatorname{fr}\left(\mathrm{K}_{j}^{P, r}(y)\right) \subset \bigcup_{j=1}^{k} \mathcal{D}_{X^{j} \cap B(y, r)}(P)$ et que $\bigcup_{j=1}^{k} \mathcal{D}_{X^{j} \cap B(y, r)}(P)$ est un représentant du germe $\Delta_{X_{y_{n}}}(P)$ par la proposition 4.4.(iii), l'hypothèse assure que la majoration suivante a lieu : $\operatorname{dim}\left(C_{Y}\left(\operatorname{fr}\left(\mathrm{K}_{j}^{P, r}(y)\right)\right)_{y_{n}}\right) \leq i-1-\operatorname{dim}(Y)$, ce qui par la proposition 4.8 donne bien : $\lim _{n \rightarrow \infty} \Theta_{i}\left(\mathbf{K}_{j}^{P, r}(y), y_{n}\right)=\Theta_{i}\left(\mathbf{K}_{j}^{P, r}(y), y\right)=\Theta_{i}\left(\mathcal{K}_{j}^{P, r}(y)\right)$.

Nous allons maintenant montrer que l'hypothèse du théorème 4.9.(ii) a lieu, c'est-à-dire que les sous-analytiques denses $\mathcal{D}^{\operatorname{dim}(Y)+1}, \cdots, \mathcal{D}^{d}$ existent bien, lorsque la stratification $\left(X^{j}\right)_{j \in\{0, \cdots, k\}}$ est $(w)$-régulière, ou encore, d'après la proposition 4.7.(iii), que les discriminants généraux sont équisécables le long de strates de Verdier.

On considère pour cela la situation suivante : $X$ est un ensemble sous-analytique fermé de $\mathbb{R}^{n}$ de dimension $d$, muni d'une stratification $(w)$-régulière $\left(X^{j}\right)_{j \in\{0, \ldots, k\}}$ dont $Y$ est une strate. On s'intéresse aux propriétés locales de la stratification au voisinage d'un point de $Y$. Au voisinage de ce point, l'adhérence de $X \backslash Y$ est $X$. Quitte à faire un changement de coordonnées locales, on peut supposer que ce point est l'origine et que $Y$ est un sous-espace vectoriel de $\mathbb{R}^{n}$. Pour $i \operatorname{de} \operatorname{dim}(Y)+1$ à $d$, et un plan $P$ de dimension $i$ contenant $Y$, on considère la projection orthogonale de noyau $P^{\perp}$ sur $P$. On note $\mathrm{N}$ le sous-espace vectoriel de dimension $n-\operatorname{dim}(Y)$ normal à $Y$ et on identifie $\mathbb{R}^{n}$ avec le produit $Y \times \mathrm{N}$. On note $G(j, N)$ la grassmannienne des $j$-plans vectoriels de $N$.

$\grave{A}$ un morphisme sous-analytique lisse $f: Z \longrightarrow \mathbb{R}$ défini sur un ensemble sousanalytique de dimension $d$ dans $\mathbb{R}^{n}$, on associe le fibré conormal relatif $T_{f}^{*} \mathbb{R}^{n}$, ensemble sous-analytique lisse de dimension $n$ du fibré cotangent $T^{*} \mathbb{R}^{n}$. Pour un morphisme sousanalytique $f: Z \longrightarrow \mathbb{R}$ lisse sur un ouvert partout dense $U$ de $Z$, le conormal relatif $T_{f}^{*} \mathbb{R}^{n}$ est, par définition, l'adhérence de $T_{\left.f\right|_{U}}^{*}$ dans $T^{*} \mathbb{R}^{n}$. Cet ensemble est stable par les homothéties réelles du fibré vectoriel cotangent $T^{*} \mathbb{R}^{n}$. Lorsque $f$ est constante sur $Z$, on note $T_{Z}^{*} \mathbb{R}^{n}$ au lieu de $T_{f}^{*} \mathbb{R}^{n}$. Lorsqu'il n'y a pas d'ambiguïté, on abrège $T_{f}^{*} \mathbb{R}^{n}$ en $T_{f}^{*}$.

ThÉORÈme 4.10. - Soit $X$ un ensemble sous-analytique fermé de $\mathbb{R}^{n}$ de dimension d, muni d'une stratification $(w)$-régulière $\left(X^{j}\right)_{j \in\{0, \ldots, k\}}$ dont $Y$ est une strate. Pour $i$ de $\operatorname{dim}(Y)+1 \grave{a}$ $d$, les trois propriétés suivantes sont vraies :

(i) Il existe un ouvert sous-analytique partout dense $\mathcal{D}^{i}$ dans $G(i-\operatorname{dim}(Y), \mathrm{N})$ tel que, pour $P$ dans $\mathcal{D}^{i}$, le plan $P^{\perp}$ ne rencontre le cône normal $C_{Y}\left(\mathcal{P}_{X}(P)\right)$ qu'à l'origine.

(ii) Il existe un ouvert sous-analytique partout dense $\mathcal{D}^{i}$ dans $G(i-\operatorname{dim}(Y), \mathrm{N})$ et un ouvert sous-analytique partout dense $\mathcal{D}^{n-i+1}$ dans $G(n-i+1, \mathrm{~N})$, tels que, pour $P$ (contenant $Y$ ) dans $\mathcal{D}^{i}$ et $W$ (orthogonal à $Y$ ) dans $\mathcal{D}^{n-i+1}$, le plan $W$ ne rencontre le cône normal $C_{Y}\left(\mathcal{P}_{X}(P)\right)$ qu'à l'origine.

(iii) Il existe un ouvert sous-analytique partout dense $\mathcal{A}^{i}$ dans la variété des drapeaux $F(1, i-$ $\operatorname{dim}(Y), \mathrm{N})$ tel que pour $(L \subset P)$ élément de $\mathcal{A}^{i}, L$ normal à $Y$ dans $P$, le plan $\pi_{P}^{-1}(L)$ ne rencontre le cône normal $C_{Y}\left(\mathcal{P}_{X}(P)\right)$ qu'à l'origine. 
Démonstration. - On considère la déformation $u: \mathcal{D}(X, Y) \longrightarrow \mathbb{R}$ de $X$ au cône normal à $Y$, naturellement plongée dans le produit $T_{Y} \mathbb{R}^{n} \times \mathbb{R}$. Le conormal relatif de cette déformation s'identifie à la déformation $\mathcal{D}\left(T_{X}^{*} \mathbb{R}^{n}, T_{Y}^{*} \mathbb{R}^{n}\right)$ de $T_{X}^{*} \mathbb{R}^{n}$ au cône normal à $T_{Y}^{*} \mathbb{R}^{n}$, ellemême sous espace du produit $T^{*}\left(T_{Y} \mathbb{R}^{n}\right) \times \mathbb{R}$. Nous noterons $\mathfrak{X}$ (resp. T) l'espace total de la déformation de $X$ au cône normal à $Y$ (resp. de la déformation de $T_{X}^{*} \mathbb{R}^{n}$ au cône normal à $T_{Y}^{*} \mathbb{R}^{n}$ ) et sa fibre spéciale $\mathfrak{X}_{0}$ (resp. $\left.\mathfrak{T}_{0}\right)$. La fibre $\mathfrak{T}_{0}$ est un sous-ensemble de $T^{*}\left(T_{Y} \mathbb{R}^{n}\right)$. On désigne par $\pi$ la projection naturelle du fibré normal $T_{Y} \mathbb{R}^{n}$ sur $Y$. Elle induit une projection pr de $T^{*}\left(T_{Y} \mathbb{R}^{n}\right)$ sur le fibré cotangent relatif $T^{*} \pi$. D'autre part, l'isomorphisme hamiltonien identifie le cône normal à $T_{Y}^{*} \mathbb{R}^{n}$ dans $T^{*} \mathbb{R}^{n}$ au fibré cotangent $T^{*}\left(T_{Y}^{*} \mathbb{R}^{n}\right)$. Si on désigne par $\pi^{\prime}$ la projection naturelle du fibré conormal $T_{Y}^{*} \mathbb{R}^{n}$ sur $Y$, elle induit à son tour une projection $\operatorname{pr}^{\prime}$ de $T^{*}\left(T_{Y}^{*} \mathbb{R}^{n}\right)$ sur $T^{*} \pi^{\prime}$. On remarque que $\mathrm{pr}^{\prime}$ s'identifie à pr et $T^{*} \pi^{\prime}$ à $T^{*} \pi$ via l'isomorphisme hamiltonien.

L'énoncé qui suit a été prouvé, lorsque $Y$ est un point par Kashiwara dans [38] dans le cadre analytique complexe.

Lemme 4.11. - L'ensemble $\operatorname{pr}\left(\mathfrak{T}_{0}\right)$ est un sous-ensemble relativement isotrope et homogène du cotangent relatif $T^{*} \pi$.

Preuve du lemme 4.11. - Le résultat se démontre localement en un point lisse de la fibre $\mathfrak{T}_{0}$ de $\mathfrak{T}$ dont la projection dans $T_{Y} \mathbb{R}^{n}$ (resp. $T_{Y}^{*} \mathbb{R}^{n}$ ) n'est pas dans la section nulle de $T_{Y} \mathbb{R}^{n}$ (resp. $T_{Y}^{*} \mathbb{R}^{n}$ ). Grâce au lemme d'aile ([15], [52]), on se ramène au cas où $\mathfrak{T}_{0}$ est de codimension 1 dans $\mathfrak{T}$. Dans ce cas, l'aile est une paramétrisation locale $\varphi$ de $\mathfrak{T}, \varphi: \mathfrak{T}_{0} \times(0, \alpha) \longrightarrow \mathfrak{T}$ telle que $\varphi(s, t)=(\varphi(s, 0), \rho(s, t))$.

On choisit des coordonnées locales $(y, x)$ dans $\mathbb{R}^{n}$ de telle sorte que $Y$ est défini par l'idéal $(x)$. On note $(\eta, \xi)$ les coordonnées correspondantes sur les fibres du fibré cotangent $T^{*} \mathbb{R}^{n}$. Sur $T^{*}\left(T_{Y} \mathbb{R}^{n}\right)$ on en déduit des coordonnées $(y, \ell, \nu, \xi)$ qui s'étendent en des coordonnées $(y, \ell, \nu, \xi, u)$ sur le produit $T^{*}\left(T_{Y} \mathbb{R}^{n}\right) \times \mathbb{R}$. L'application :

$$
(y, \ell, u) \longmapsto(y, u \ell, u)
$$

induit un isomorphisme de $\mathfrak{X} \backslash \mathfrak{X}_{0}$ sur $X \times \mathbb{R}_{+}^{*}$. Elle se relève en une application :

$$
(y, \ell, \nu, \xi, u) \longmapsto(y, u \ell, u \nu, \xi, u)
$$

qui induit un isomorphisme de $\mathfrak{T} \backslash \mathfrak{T}_{0}$ sur $T_{X}^{*} \mathbb{R}^{n} \times \mathbb{R}_{+}^{*}$. Par définition de $T_{X}^{*} \mathbb{R}^{n}$, la forme différentielle $\eta d y+\xi d x$ est nulle en restriction à la partie lisse de $T_{X}^{*} \mathbb{R}^{n}$. On en déduit l'égalité suivante, vraie en restriction aux points lisses de $\mathfrak{T} \backslash \mathfrak{T}_{0}$ :

$$
\frac{\xi}{\|\xi\|\|\ell\|} d \ell=-\frac{\nu}{\|\xi\|\|\ell\|} d y-\frac{\langle\xi \cdot \ell\rangle}{\|\xi\|\|\ell\|} \frac{d u}{u} .
$$

La condition $(w)$ se traduit par le fait que la quantité :

$$
\frac{\|\nu\|}{\|\xi\|\|\ell\|}
$$

est localement bornée. La condition $\left(b^{\prime}\right)$, conséquence de $(w)$, se traduit par le fait que la quantité :

$$
\frac{\langle\xi \cdot \ell\rangle}{\|\xi\|\|\ell\|}
$$


tend vers 0 avec $t$. La forme différentielle $\frac{\langle\xi \cdot \ell\rangle}{\|\xi\|\|\ell\|} \frac{d u}{u}$ s'étend donc en une forme sur $\mathfrak{T}$ au voisinage des points considérés. On en conclut que la forme différentielle $\frac{\xi}{\|\xi\|\|\ell\|} d \ell$ induit une forme différentielle relative au-dessus de $Y$, nulle sur la partie lisse de $\operatorname{pr}\left(\mathfrak{T}_{0}\right)$.

Fin de la preuve du théorème 4.10. - Considérons le fibré cotangent relatif $T^{*} \pi$ et sa projection canonique $p \operatorname{sur} T_{Y} \mathbb{R}^{n}$. L'isomorphisme hamiltonien identifie $T^{*} \pi$ au fibré cotangent relatif $T^{*} \pi^{\prime}$ de la projection $\pi^{\prime}: T_{Y}^{*} \mathbb{R}^{n} \longrightarrow Y$. On a donc également une projection canonique $p^{\prime}$ de $T^{*} \pi$ sur $T_{Y}^{*} \mathbb{R}^{n}$. Un plan $P$ de dimension $i$ contenant $Y$ étant donné dans $\mathbb{R}^{n}$, il induit, grâce à la structure euclidienne de $\mathbb{R}^{n}$, un sous-fibré de $\operatorname{rang} i-\operatorname{dim}(Y)$ de $T_{Y}^{*} \mathbb{R}^{n}$, noté encore $P$. D'autre part, l'orthogonal $P^{\perp}$ induit un sous-fibré de rang $n-i$ de $T_{Y} \mathbb{R}^{n}$, noté encore $P^{\perp}$. Considérons le produit fibré $P^{\perp} \times_{Y} P$. Le lemme 4.11 a les conséquences suivantes : il existe un ouvert $\mathcal{D}^{i}$ partout dense dans la grassmannienne $G(i-\operatorname{dim}(Y), \mathrm{N})$ tel que pour $V$ de dimension $i$ contenant $Y$,

- l'intersection de $P^{\perp} \times_{Y} P$ avec tout sous-ensemble relativement isotrope de $T^{*} \pi$, en particulier avec $\operatorname{pr}\left(\mathfrak{T}_{0}\right)$ est réduite à la section nulle de $T^{*} \pi$;

- la projection $p\left(p^{-1}(P) \cap \operatorname{pr}\left(\mathfrak{T}_{0}\right)\right)$ est le cône normal à la polaire $\mathcal{P}_{X}(P)$.

Nous venons de montrer la première assertion du théorème. Pour terminer la preuve, il suffit de vérifier que les trois assertions sont en fait équivalentes. On considère un espace vectoriel $E$ de dimension $k$ et la variété des drapeaux $\ell \subset H$ formés d'une droite $\ell$ et d'un hyperplan $H$ de $E$. On la note $\mathcal{F}$. Elle est contenue dans le produit $\mathbb{P} \times \mathbb{P}^{\vee}$ du projectif des droites par le projectif des hyperplans de $E$. Si on se donne un drapeau :

$$
\mathbf{D}:\{0\}=V_{0} \subset V_{1} \subset \cdots \subset V_{k-1} \subset V_{k}=E,
$$

les cycles $\sigma_{i}(\mathbf{D}): \ell \subset V_{i} \subset H$ pour $i$ de 1 à $k-1$ engendrent la cohomologie de $\mathcal{F}$ en codimension $k-1$. Il en est de même des cycles :

$$
\varsigma_{i}(\mathbf{D}): \ell \subset V_{i+1}, V_{i} \subset H
$$

pour $i$ de 1 à $k-1$, puisque dans la cohomologie de $\mathcal{F}$ on a $: \varsigma_{i}(\mathbf{D})=\sigma_{i}(\mathbf{D})+\sigma_{i+1}(\mathbf{D})$ pour $i$ de 1 à $k-1$.

Donnons-nous un autre drapeau $\mathbf{D}^{\prime}: W_{1} \subset \cdots \subset W_{k}=E$ de sous-espaces vectoriels de $E$. La variété de drapeaux $\mathcal{F}$ est une sous-variété de codimension 1 dans le produit $\mathbb{P} \times \mathbb{P}^{\vee}$ où la cohomologie en codimension $k-1$ est engendrée par les cycles

$$
\varsigma_{i}\left(\mathbf{D}, \mathbf{D}^{\prime}\right): \ell \subset W_{i+1}, V_{i} \subset H
$$

pour $i$ de 0 à $k-1$. Pour un sous-ensemble $\Sigma$ de $\mathcal{F}$, les quatre propriétés suivantes sont donc équivalentes :

1. $\Sigma$ est de dimension strictement inférieure à $k-1$.

2. Il existe un ouvert partout dense de drapeaux tel que pour tout drapeau $\mathbf{D}$ de cet ouvert et $i$ de 1 à $k-1$, l'intersection de $\Sigma$ avec les $\sigma_{i}(\mathbf{D})$ est vide. 
3. Il existe un ouvert partout dense des couples de drapeaux tel que pour tout couple de drapeaux $\left(\mathbf{D}, \mathbf{D}^{\prime}\right)$ de cet ouvert et $i$ de 0 à $k-1$, l'intersection de $\Sigma$ avec les $\varsigma_{i}\left(\mathbf{D}, \mathbf{D}^{\prime}\right)$ est vide.

4. Il existe un ouvert partout dense de drapeaux tel que pour tout drapeau $\mathbf{D}$ de cet ouvert et $i$ de 1 à $k-1$, l'intersection de $\Sigma$ avec les $\varsigma_{i}(\mathbf{D})$ est vide.

Remarque 4.12. - On note que la condition 4.10.(i) est équivalente à la condition $\left(b^{*}\right)$ le long de $Y$ et à la suivante :

4.10.(iv) : Il existe un ouvert sous-analytique partout dense $\mathcal{G}^{i}$ dans $G(i-\operatorname{dim}(Y), \mathrm{N})$ tel que, pour $P$ dans $\mathcal{G}^{i}, P$ vérifie la condition $(*)$ introduite au début de la section 4.

D'autre part tout sous-ensemble isotrope de $\mathbb{P} \times \mathbb{P}^{\vee}$ étant de dimension strictement inférieure à $k-1$, la conclusion du lemme 4.11 implique chacune des conditions équivalentes 4.10.(i) à 4.10.(iv). Nous ne savons pas si la réciproque est vraie.

\section{Appendice}

\section{Polyèdres et valuations sphériques - Calcul des coefficients $m_{i}^{j}$}

Nous traitons ici le cas où l'ensemble $X_{0}$ (noté $V$ ) est un cône polyédral de $\mathbb{R}^{n}$ de sommet l'origine, de dimension maximale et nous expliquons comment le théorème 3.1 se rattache dans ce contexte à des questions de géométrie convexe sphérique. Le cas conique polyédral permet en outre un calcul aisé des coefficients $m_{i}^{j}$ de la matrice $\mathcal{M}$ du théorème 3.1 .

Par polyèdre de $\mathbb{R}^{n}$ nous entendons une partie de $\mathbb{R}^{n}$ qui est une intersection finie de demiespaces fermés. Un polyèdre de $\mathbb{R}^{n}$ est ainsi une partie convexe de $\mathbb{R}^{n}$. Un cône polyédral de $\mathbb{R}^{n}$ de sommet l'origine est un polyèdre de $\mathbb{R}^{n}$ obtenu comme intersection de demi-espaces fermés contenant tous dans leur bord l'origine. Par polytope de $\mathbb{R}^{n}$ nous entendons un polyèdre compact de $\mathbb{R}^{n}$, c'est-à-dire l'enveloppe convexe d'un nombre fini de points de $\mathbb{R}^{n}$. Enfin un polytope sphérique (de $S^{n-1}$ ) est l'intersection d'un cône polyédral (de $\mathbb{R}^{n}$ ) de sommet l'origine et de la sphère $S^{n-1}$. Nous noterons $\mathcal{K}^{n}$ l'ensemble des convexes compacts de $\mathbb{R}^{n}$ et $\mathcal{K} S^{n-1}$ l'ensemble des convexes compacts de $S^{n-1}$.

Nous introduisons maintenant brièvement la notion de valuations sur les convexes. Pour plus de détails on pourra se reporter à [68, 69] ou [55].

Une application $v: \mathcal{K}^{n} \rightarrow \mathbb{R}$ (resp. $v: \mathcal{K} S^{n-1} \rightarrow \mathbb{R}$ ) est une valuation réelle (resp. une valuation sphérique réelle) si $v(\varnothing)=0$ et si quels que soient $K, L \in \mathcal{K}^{n}$ (resp. $K, L \in \mathcal{K} S^{n-1}$ ) tels que $K \cup L \in \mathcal{K}^{n}$ (resp. $K \cup L \in \mathcal{K} S^{n-1}$ ), on a :

$$
v(K \cup L)=v(K)+v(L)-v(K \cap L)
$$

On dit qu'une valuation $v$ sur $\mathcal{K}^{n}$ (resp. $\mathcal{K} S^{n-1}$ ) est continue si elle est continue pour la métrique de Hausdorff sur $\mathcal{K}^{n}$ (resp. sur $\mathcal{K} S^{n-1}$ ). On dit qu'une valuation $v$ sur $\mathcal{K}^{n}$ (resp. $\mathcal{K} S^{n-1}$ ) est simple si la restriction de $v$ aux convexes de dimension non maximale est nulle. Soit Iso ${ }^{n}$ le groupe des isométries de $\mathbb{R}^{n}$. Ce groupe agit sur $\mathcal{K}^{n}$, de même que le groupe $\mathcal{O}^{n}$ des isométries de la sphère agit $\operatorname{sur} \mathcal{K} S^{n-1}$. On dit qu'une valuation $v \operatorname{sur} \mathcal{K}^{n}\left(\operatorname{resp} . \mathcal{K} S^{n-1}\right.$ ) est invariante (sous l'action de $\mathrm{Iso}^{n}$, resp. sous l'action de $\mathcal{O}^{n}$ ) si quels que soient $K \in \mathcal{K}^{n}$ (resp. $K \in \mathcal{K} S^{n-1}$ ), $g \in$ Iso $^{n}$ (resp. $g \in \mathcal{O}^{n}$ ), on a $: v(g . K)=v(K)$. 
Soient $i, j \in\{0, \cdots, n\}$. Si $C$ est un cône convexe sous-analytique de $\mathbb{R}^{n}$ de sommet l'origine, par $i$-homogénéité de $\Lambda_{i}$ et par convexité, on a :

$$
\begin{aligned}
\Lambda_{i}^{\text {loc }}\left(C_{0}\right) & =\frac{1}{\alpha_{i}} \cdot \Lambda_{i}\left(C \cap B_{(0,1)}^{n}\right) \\
& \left.=\frac{1}{\alpha_{i} \cdot \beta(n, i)} \cdot \bar{\gamma}_{n-i, n}\left(\left\{\bar{P} \in \bar{G}(n-i, n) ; \bar{P} \cap C \cap B_{(0,1)}^{n}\right) \neq \varnothing\right\}\right) \\
\text { et } \sigma_{j}\left(C_{0}\right) & =\int_{P \in G(j, n)} \Theta_{j}\left(\left(\pi_{P}(C)\right)_{0}\right) d \gamma_{j, n}(P) .
\end{aligned}
$$

Notons $\widehat{\Lambda}_{i}^{\text {loc }}$ et $\widehat{\sigma}_{j}$ les valuations sphériques sur $\mathcal{K} S^{n-1}$, auxquelles donnent lieu $\Lambda_{i}^{\text {loc }}$ et $\sigma_{j}$ :

$$
\forall K \in \mathcal{K} S^{n-1}, \quad \widehat{\Lambda_{i}}(K)=\Lambda_{i}^{\text {loc }}\left(\widehat{K}_{0}\right) \text { et } \quad \widehat{\sigma_{j}}(K)=\sigma_{j}\left(\widehat{K}_{0}\right),
$$

où $\widehat{K}$ est le cône dans $\mathbb{R}^{n}$ sur $K$ de sommet l'origine. Ces valuations sont continues et invariantes sous l'action de $\mathcal{O}^{n}$.

En tant qu'objet d'étude systématique l'introduction des valuations vues comme invariants de découpage remonte au troisième problème de Hilbert (voir [4], [63]). Cette étude culmine dans la caractérisation, due à Hadwiger ([28], [39]), des valuations invariantes et continues :

THÉORÈME ([28], [39]). - Si v est une valuation sur $\mathcal{K}^{n}$ continue et invariante sous l'action de Iso $^{n}$, il existe des constantes réelles $\alpha_{0}, \cdots, \alpha_{n}$ telles que $: v=\sum_{i=0}^{n} \alpha_{i} \cdot \Lambda_{i}$.

Une façon équivalente d'énoncer le théorème d'Hadwiger est la suivante :

Si $v$ est une valuation simple sur $\mathcal{K}^{n}$, continue et invariante sous l'action de $\mathrm{Iso}^{n}$, il existe une constante réelle $\alpha$ telle que : $v=\alpha \cdot \Lambda_{n}=\alpha \cdot \mathcal{H}^{n}$.

Un problème apparemment délicat consiste à savoir si le théorème d'Hadwiger admet un équivalent sphérique :

Question ([27, Problème 74], [9, Problème 14.3]). - Si v est une valuation simple sur $\mathcal{K} S^{n-1}$, continue et invariante sous l'action de $\mathcal{O}^{n}$, est-il vrai que $v$ est proportionnelle au volume $\mathcal{H}^{n-1}$ de $S_{(0,1)}^{n-1}$ ?

Une réponse positive à cette question aurait pour corollaire immédiat le théorème 3.1 et inversement la preuve du théorème 3.1 est une réponse positive à la question de la validité de la version sphérique du théorème d'Hadwiger, dans le cas particulier des valuations $\widehat{\sigma}_{*}$ et $\widehat{\Lambda}_{*}$. Notons que l'on dispose d'une réponse positive à cette question, dans le cas où $n \leq 3$ (cf. [55, Théorème 14.4]) et dans le cas où la valuation est de signe constant. Dans ce dernier cas la continuité de la valuation n'est pas requise et la valuation est définie a priori sur les polytopes convexes et non pas nécessairement sur tous les convexes de $S^{n-1}$ :

THÉORÈme A.1 ([66, Théorème 6.2], [67]). - Soit v une valuation simple définie sur les polytopes de $S^{n-1}$, invariante sous l'action de $\mathcal{O}^{n}$ et à valeurs dans $\mathbb{R}_{+}$. Il existe alors $c \in \mathbb{R}_{+}$tel que $v=c \cdot \mathcal{H}_{\mid S^{n-1}}^{n-1}$. 
Venons-en maintenant au calcul des coefficients de la matrice $\mathcal{M}$ du théorème 3.1.

Si $V$ est un polyèdre de $\mathbb{R}^{n}$ de dimension $n$, on dit qu'un hyperplan qui le borde est une facette de $V$. Le vecteur normal à une facette $F$ de $V$ est le vecteur unitaire orthogonal à $F$ situé dans le demi-espace défini par $F$ qui ne contient pas $V$. Pour $i \in\{0, \cdots, n-1\}$, une $i$-face de $V$ est l'intersection avec $V$ de $n-i$ facettes distinctes de $V$. On note $\mathcal{F}_{i}(V)$ l'ensemble des $i$-faces de $V$. Par convention $\mathcal{F}_{n}(V)=\{V\}$. Si $V$ est un polytope sphérique, pour $i \in$ $\{0, \cdots, n-1\}$, une $i$-face de $V$ est définie comme étant l'intersection de $S^{n-1}$ et d'une $(i+1)$ face de $\widehat{V}$. À tout point $x \in V$ on peut associer $F_{x}$, l'unique face de $V$ de dimension minimale contenant $x$. Si $x \in \partial V$ (le bord de $V$ ), on définit $C(x, V)$, le cône conormal à $V$ en $x$, de la façon suivante : $C(x, V)$ est le cône positif de $\mathrm{T}_{x} \mathbb{R}^{n}$ engendré par les vecteurs normaux aux facettes de $V$ contenant $x$. On convient que : $C(x, V)=\{0\}$, lorsque $x \in V \backslash \partial V$.

Remarque. - Si $V$ est un polyèdre de $\mathbb{R}^{n}, x \in V$, et si $F_{x}$ est de dimension $i \in$ $\{0, \cdots, n-1\}, C(x, V)$ est un cône de dimension $n-i$. De plus quel que soit $y \in F_{x}$, $C(x, V)=C(y, V)$. On définit donc le cône conormal de $V$ le long d'une face $F$ de $V$ par: $C(F, V)=C(x, V)$, où $x$ est quelconque dans $F$. On a : $C(V, V)=\{0\}$.

Si $V$ est un polyèdre dégénéré de $\mathbb{R}^{n}$, c'est-à-dire si le sous-espace vectoriel $[V]$ de $\mathbb{R}^{n}$ engendré par $V$ est de dimension $<n$, on note $C_{[V]}(x, V)$ le cône conormal de $V$ en $x$ dans $[V]$, au sens de la définition qui précède, puisque $V$ est de codimension nulle dans $[V]$. Avec cette notation, le cône conormal de $V$ en $x$ dans $\mathbb{R}^{n}$, noté $C_{\mathbb{R}^{n}}(x, V)$ est défini par $C_{[V]}(x, V) \times$ $[V]^{\perp}$. Le cône conormal est ainsi relatif à l'espace dans lequel il est obtenu, mais nous donnons une définition intrinsèque attachée au cône conormal : l'angle extérieur.

Définition A.2. - Soient $V$ un polyèdre $\mathbb{R}^{n}$ et $F \in \mathcal{F}_{i}(V)$. On définit $\gamma(F, V)$, l'angle extérieur de $V$ en $F$ par:

$$
\gamma(F, V)=\frac{1}{\alpha_{n-i}}\left(\mathcal{H}^{n-i}\left(C(F, V) \cap B_{(0,1)}^{n}\right)\right)=\Theta_{n-i}\left(C(F, V)_{0}\right) .
$$

Par convention $\gamma(V, V)=1$ (ce qui, compte tenu de $C(V, V)=\{0\}$, signifie que l'on opte pour la convention $\left.\Theta_{0}(\{0\})=1\right)$.

Remarque. - Comme la densité d'un produit est le produit des densités des facteurs (dans les dimensions adéquates), lorsque $V$ est un polyèdre dégénéré de $\mathbb{R}^{n}$, l'égalité $C_{\mathbb{R}^{n}}(x, V)=C_{[V]}(x, V) \times[V]^{\perp}$ montre que l'angle extérieur de $V$ en l'une de ses faces ne dépend pas de l'espace ambiant dans lequel on le calcule.

La formule cinématique principale donne l'expression de $\Lambda_{i}$ à l'aide des angles extérieurs attachés aux $i$-faces :

THÉORÈME (formule cinématique principale [69, 4.5.2], [68, 7.2])

Soit $V$ un polytope de $\mathbb{R}^{n} ;$ pour tout $i \in\{0, \cdots, n\}$, on $a$ :

$$
\Lambda_{i}(V)=\sum_{F \in \mathcal{F}_{i}} \gamma(F, V) \cdot \mathcal{H}^{i}(F)
$$


Le théorème d'Hadwiger implique alors que toute valuation $v$ sur $\mathcal{K}^{n}$, continue et invariante sous l'action de Iso ${ }^{n}$, vérifie :

(*) $\quad \exists \alpha_{1}, \cdots, \alpha_{n} \in \mathbb{R}, \quad \forall V$ polytope de $\mathbb{R}^{n}, \quad v(V)=\sum_{i=0}^{n} \alpha_{i} \sum_{F \in \mathcal{F}_{i}} \gamma(F, V) \cdot \mathcal{H}^{i}(F)$

Bien que nous ne sachions pas si l'équivalent sphérique du théorème d'Hadwiger est vrai, nous allons montrer, pour prouver le théorème 3.1 sur les cônes polyédraux, que les valuations $\widehat{\Lambda}_{i}$ et $\widehat{\sigma}_{j}$ sur les polytopes de $S^{n-1}$ sont des combinaisons linéaires des équivalents sphériques des $\gamma(F, V) \cdot \mathcal{H}^{i}(F)$.

Soient $V$ un polytope de $S^{n-1}$ et $\widehat{V}$ le cône polyédral de sommet l'origine de $\mathbb{R}^{n}$ qui lui est associé. Si $F$ est une $k$-face de $V$, on note $\widehat{F}$ la $(k+1)$-face de $\widehat{V}$ qui lui est associée. Nous allons prouver qu'existent des constantes $a_{0}, \cdots, a_{n-1}, b_{0}, \cdots, b_{n-1}$ indépendantes de $V$ telles que :

$(* *)$

$$
\widehat{\Lambda}_{i}(V)=\sum_{k=0}^{n-1} a_{k} \sum_{F \in \mathcal{F}_{k}(V)} \gamma(\widehat{F}, \widehat{V}) \cdot \mathcal{H}^{k}(F) \text { et } \widehat{\sigma}_{j}(V)=\sum_{k=0}^{n-1} b_{k} \sum_{F \in \mathcal{F}_{k}(V)} \gamma(\widehat{F}, \widehat{V}) \cdot \mathcal{H}^{k}(F) .
$$

En prouvant $(* *)$ nous allons ainsi prouver l'équivalent sphérique de $(*)$ pour les valuations sphériques particulières que sont $\widehat{\Lambda}_{i}$ et $\widehat{\sigma}_{j}, i, j \in\{0, \cdots, n\}$, c'est-à-dire répondre positivement au problème 15.5 de [55] pour les valuations $\widehat{\Lambda}_{i}$. Remarquons que dans les égalités $(* *)$, $\mathcal{H}^{k}(F)=\mathcal{H}^{k}\left(S^{k}\right) \cdot \Theta_{k+1}\left(\widehat{F}_{0}\right)$.

Lemme A.3. - Soit $V$ un cône polyédral de $\mathbb{R}^{n}$ de sommet l'origine. On a :

$$
\sum_{k=0}^{n} \sum_{F \in \mathcal{F}_{k}(V)} \gamma(F, V) \cdot \Theta_{k}\left(F_{0}\right)=1
$$

Démonstration. - Soit $F \in \mathcal{F}_{k}(V)$; le cône $F+C(F, V)$ est de dimension $n$ et sa densité en l'origine est $\Theta_{n}\left(\left(F+C(F, V)_{0}\right)\right)=\Theta_{k}\left(F_{0}\right) \cdot \Theta_{n-k}\left((C(F, V))_{0}\right)=\Theta_{k}\left(F_{0}\right) \cdot \gamma(F, V)$. Or comme $V$ est convexe, $\bigcup_{k=0}^{n} \bigcup_{F \in \mathcal{F}_{k}(V)} F+C(F, V)=\mathbb{R}^{n}$ et comme, lorsque $F \neq G$, $(F+C(F, V)) \cap(G+C(G, V))$ est un cône de dimension $<n$, on en déduit que :

$$
\Theta_{n}\left(\left(\bigcup_{k=0}^{n} \bigcup_{F \in \mathcal{F}_{k}(V)} F+C(F, V)\right)_{0}\right)=\sum_{k=0}^{n} \sum_{F \in \mathcal{F}_{k}(V)} \Theta_{n}\left((F+C(F, V))_{0}\right)=1
$$

ThÉorème A.4. - Soit $j \in\{0, \cdots, n\}$ et soit $V$ un cône polyédral de $\mathbb{R}^{n}$ de sommet l'origine. On a alors :

$$
\sigma_{j}\left(V_{0}\right)=\sum_{k=j}^{n} \sum_{F \in \mathcal{F}_{k}(V)} \gamma(F, V) \cdot \Theta_{k}\left(F_{0}\right)
$$


Démonstration. - Par définition $\sigma_{j}\left(V_{0}\right)=\int_{P \in G(j, n)} \Theta_{j}\left(\left(\pi_{P}(V)\right)_{0}\right) d \gamma_{j, n}(P)$. Par le lemme A.3 on obtient :

$$
\sigma_{j}\left(V_{0}\right)=1-\int_{P \in G(j, n)} \sum_{k=0}^{j-1} \sum_{G \in \mathcal{F}_{k}\left(\pi_{P}(V)\right)} \gamma\left(G, \pi_{P}(V)\right) \cdot \Theta_{k}\left(G_{0}\right) d \gamma_{j, n}(P) .
$$

Soient $P \in G(j, n)$ générique et $k \in\{0, \cdots, j-1\}$. Pour toute $k$-face $F$ de $V, \pi_{P}(F)$ est une $k$-face de $\pi_{P}(V)$ si et seulement si $P \cap C(F, V) \neq\{0\}$ et dans ce cas :

$$
C\left(\pi_{P}(F), \pi_{P}(V)\right)=P \cap C(F, V) .
$$

Réciproquement à toute $k$-face $G$ de $\pi_{P}(V)$ on peut associer une unique $k$-face $F$ de $V$ telle que $\pi_{P}(F)=G$. On peut donc écrire :

$$
\sigma_{j}\left(V_{0}\right)=1-\int_{P \in G(j, n)} \sum_{k=0}^{j-1} \sum_{F \in \mathcal{F}_{k}(V)} \Theta_{j-k}\left((C(F, V) \cap P)_{0}\right) \cdot \Theta_{k}\left(\left(\pi_{P}(F)\right)_{0}\right) d \gamma_{j, n}(P) .
$$

Soit alors $[F]$ l'espace vectoriel engendré par $F$. On définit une application $v$ sur les polytopes sphériques de $[F]$ de la façon suivante. Pour tout polytope sphérique $W$ de $[F]$ :

$$
v(W)=\int_{P \in G(j, n)} \Theta_{j-k}\left((C(F, V) \cap P)_{0}\right) \cdot \Theta_{k}\left(\left(\pi_{P}(\widehat{W})\right)_{0}\right) d \gamma_{j, n}(P) .
$$

L'application $v$ est une valuation simple sur les polytopes sphériques de $[F]$, invariante sous l'action des rotations de $[F]$. Comme de plus $v$ est positive, par le théorème A.1, $v$ est proportionnelle au volume $\mathcal{H}^{k-1}$ sur la sphère unité de $[F]$. Il existe $c \in \mathbb{R}_{+}$, tel que pour tout polytope sphérique $W$ de $[F], v(W)=c \cdot \mathcal{H}^{k-1}(W)$. En égalant $W$ à la sphère unité de $[F]$, on obtient, puisqu'alors $\Theta_{k}\left(\left(\pi_{P}(\widehat{W})\right)_{0}\right)=1$ :

$$
\int_{P \in G(j, n)} \Theta_{j-k}\left((C(F, V) \cap P)_{0}\right) d \gamma_{j, n}(P)=c \cdot \mathcal{H}^{k-1}\left(S_{(0,1)}^{k-1}\right) .
$$

On en déduit que :

$$
\begin{aligned}
v\left(F \cap S^{n-1}\right) & =\frac{\mathcal{H}^{k-1}\left(F \cap S^{n-1}\right)}{\mathcal{H}^{k-1}\left(S_{(0,1)}^{k-1}\right)} \cdot \int_{P \in G(j, n)} \Theta_{j-k}\left((C(F, V) \cap P)_{0}\right) d \gamma_{j, n}(P) \\
& =\Theta_{k}(F) \cdot \int_{P \in G(j, n)} \Theta_{j-k}\left((C(F, V) \cap P)_{0}\right) d \gamma_{j, n}(P) .
\end{aligned}
$$

Maintenant l'application $u$ définie sur les polytopes sphériques $W$ de $[F]^{\perp}$ par :

$$
u(W)=\int_{P \in G(j, n)} \Theta_{j-k}\left((\widehat{W} \cap P)_{0}\right) d \gamma_{j, n}(P)
$$

est une valuation simple, positive, invariante sous l'action des rotations de $[F]^{\perp}$. À nouveau, par le théorème A.1, $u$ est proportionnelle au volume sur la sphère unité de $[F]^{\perp}$ (on peut aussi invoquer la formule de Cauchy-Crofton sphérique, cf. [20, 3.2.48]), ce qui donne :

$$
v(F)=\Theta_{k}(F) \cdot \Theta_{n-k}\left((C(F, V))_{0}\right) .
$$


On a ainsi prouvé l'égalité :

$\sigma_{j}\left(V_{0}\right)=1-\sum_{k=0}^{j-1} \sum_{F \in \mathcal{F}_{k}(V)} \Theta_{j-k}\left((C(F, V))_{0}\right) \cdot \Theta_{k}\left(F_{0}\right)=1-\sum_{k=0}^{j-1} \sum_{F \in \mathcal{F}_{k}(V)} \gamma(F, V) \cdot \Theta_{k}\left(F_{0}\right)$.

Le théorème $\mathrm{A} .4$ résulte de cette égalité et du lemme A.3.

Nous exprimons maintenant à leur tour les invariants $\Lambda_{i}^{\text {loc }}$, dans le cas polyédral, comme combinaisons linéaires des produits des densités des $k$-faces par leur angle extérieur.

ThÉORÈme A.5. - Quels que soient $i \in\{0, \cdots, n\}$ et le cône polyédral $V$ de $\mathbb{R}^{n}$ de sommet l'origine :

$$
\Lambda_{i}^{\mathrm{loc}}\left(V_{0}\right)=\sum_{k=i}^{n} a_{i}^{k} \sum_{F \in \mathcal{F}_{k}(V)} \gamma(F, V) \cdot \Theta_{k}\left(F_{0}\right)
$$

où quel que soit $k \in\{i, \cdots, n\}, a_{i}^{k}=\frac{\alpha_{k}}{\alpha_{k-i} \cdot \alpha_{i}} C_{k}^{i}$.

Démonstration. - On a déjà remarqué que $\Lambda_{i}^{\mathrm{loc}}\left(V_{0}\right)=\frac{1}{\alpha_{i}} \cdot \Lambda_{i}\left(V \cap B_{(0,1)}^{n}\right)$. Calculons le volume du voisinage tubulaire de rayon $r$ autour de $V \cap B_{(0,1)}^{n}$ afin d'obtenir $\Lambda_{i}^{\operatorname{loc}}\left(V_{0}\right)$ :

$$
\mathcal{H}^{n}\left(\mathcal{T}_{r}\left(V \cap B_{(0,1)}^{n}\right)\right)=\sum_{i=0}^{n} \alpha_{i} \cdot \Lambda_{i}^{\mathrm{loc}}\left(V_{0}\right) \cdot \alpha_{n-i} \cdot r^{n-i}
$$

Rappelons que $C(x, V)$ désigne le cône conormal de $V$ en $x$. Si $x \in S_{(0,1)}^{n} \cap V$ et $F\left(=F_{x}\right)$ est la face de $V$ contenant $x$, nous définissons le cône conormal de $V \cap B_{(0,1)}^{n}$ en $x$ par $\mathbb{R}_{+}$. $x+C(F, V)$ et nous le notons aussi $C(x, V)$. Avec cette notation :

$$
\mathcal{T}_{r}\left(V \cap B_{(0,1)}^{n}\right)=\coprod_{x \in V \cap B_{(0,1)}^{n}} x+\left[C(x, V) \cap B_{(0, r)}^{n}\right] .
$$

Soit $F \in \mathcal{F}_{0}(V) \cup \cdots \cup \mathcal{F}_{n}(V), x \in F \cap S_{(0,1)}^{n}$ et $y \in C(F, V) \cap S_{(0,1)}^{n}$; on note (pour $r \leq 1$ ) :

$$
\mathcal{A}_{x, y}=[0,1] \cdot x+\left(([0,1] \cdot y+[0,1] \cdot x) \cap B_{(0, r)}^{n}\right) \text {. }
$$

On a alors :

$$
\mathcal{T}_{r}\left(V \cap B_{(0,1)}^{n}\right)=\coprod_{F \in \mathcal{F}_{0}(V) \cup \cdots \cup \mathcal{F}_{n}(V)} \coprod_{(x, y) \in\left(F \cap S_{(0,1)}^{n}\right) \times\left(C(F, V) \cap S_{(0,1)}^{n}\right)} \mathcal{A}_{x, y} .
$$

Si $F$ est une face de $V$ de dimension $k$, le théorème de changement de variables donne l'existence d'une constante $c_{k, n}(r)$ indépendante de $F$, telle que :

$$
\mathcal{H}^{n}\left(\coprod_{(x, y) \in\left(F \cap S_{(0,1)}^{n}\right) \times\left(C(F, V) \cap S_{(0,1)}^{n}\right)} \mathcal{A}_{x, y}\right)=c_{k, n}(r) \cdot \gamma(F, V) \cdot \Theta_{k}\left(F_{0}\right) .
$$

Si $F=\mathbb{R}^{k} \times\{0\}^{n-k}$, comme $\mathcal{H}^{n}\left(\coprod_{(x, y) \in\left(F \cap S_{(0,1)}^{n}\right) \times\left(C(F, V) \cap S_{(0,1)}^{n}\right)} \mathcal{A}_{x, y}\right)=\mathcal{H}^{n}\left(\mathcal{T}_{r}\left(B_{(0,1)}^{k}\right)\right)$, on déduit de (3) que :

$$
c_{k, n}(r)=\mathcal{H}^{n}\left(\mathcal{T}_{r}\left(B_{(0,1)}^{k}\right)\right)=\sum_{j=0}^{n} \Lambda_{j}\left(B_{(0,1)}^{k}\right) \cdot \alpha_{n-j} \cdot r^{n-j}=\sum_{j=0}^{k} \Lambda_{j}\left(B_{(0,1)}^{k}\right) \cdot \alpha_{n-j} \cdot r^{n-j} .
$$


Or les $\Lambda_{j}$ ne dépendent pas de la dimension de l'espace euclidien dans lequel on les calcule; en voyant $\mathcal{B}_{(0,1)}^{k}$ dans $\mathbb{R}^{k}$ au lieu de $\mathbb{R}^{n}$, on obtient :

$$
\alpha_{k} \cdot(1+r)^{k}=\mathcal{H}^{k}\left(B_{(0, r+1)}\right)=\mathcal{H}^{k}\left(\mathcal{T}_{r}\left(B_{(0,1)}^{k}\right)\right)=\sum_{j=0}^{k} \Lambda_{j}\left(B_{(0,1)}^{k}\right) \cdot \alpha_{k-j} \cdot r^{k-j}
$$

Cette dernière égalité donne, pour tout $j \in\{0, \cdots, k\}$ :

$$
\Lambda_{j}\left(B_{(0,1)}^{k}\right)=\frac{\alpha_{k}}{\alpha_{k-j}} \cdot C_{k}^{j}
$$

et donc :

$$
c_{k, n}(r)=\sum_{j=0}^{k} \frac{\alpha_{k} \cdot \alpha_{n-j}}{\alpha_{k-j}} \cdot C_{k}^{j} \cdot r^{n-j} .
$$

Les égalités (1), (2), (3) et (4) donnent enfin :

$$
\begin{aligned}
\mathcal{H}^{n}\left(\mathcal{T}_{r}\left(V \cap B_{(0,1)}^{n}\right)\right) & =\sum_{k=0}^{n}\left(\sum_{j=0}^{k} \frac{\alpha_{k} \cdot \alpha_{n-j}}{\alpha_{k-j}} \cdot C_{k}^{j} \cdot r^{n-j}\right) \sum_{F \in \mathcal{F}_{k}} \gamma(F, V) \cdot \Theta_{k}\left(F_{0}\right) \\
& =\sum_{i=0}^{n}\left(\sum_{k=i}^{n} \frac{\alpha_{k}}{\alpha_{k-i} \cdot \alpha_{i}} C_{k}^{i} \sum_{F \in \mathcal{F}_{k}} \gamma(F, V) \cdot \Theta_{k}\left(F_{0}\right)\right) \alpha_{n-i} \cdot \alpha_{i} \cdot r^{n-i}
\end{aligned}
$$

c'est-à-dire :

$$
\Lambda_{i}^{\mathrm{loc}}\left(V_{0}\right)=\sum_{k=i}^{n} \frac{\alpha_{k}}{\alpha_{k-i} \cdot \alpha_{i}} C_{k}^{i} \sum_{F \in \mathcal{F}_{k}} \gamma(F, V) \cdot \Theta_{k}\left(F_{0}\right)
$$

Des théorèmes A.4 et $\mathrm{A} .5$ on déduit immédiatement les valeurs des coefficients $m_{i}^{j} \mathrm{du}$ théorème 3.1 :

Calcul des coefficients $m_{i}^{j}$ du théorème 3.1. - Soit $V$ un cône polyédral de $\mathbb{R}^{n}$ de sommet l'origine et soit $k \in\{0, \cdots, n\}$. Le théorème A.4 donne :

$$
\begin{gathered}
\sum_{F \in \mathcal{F}_{k}} \gamma(F, V) \cdot \Theta_{k}\left(F_{0}\right)=\sigma_{k}\left(V_{0}\right)-\sigma_{k+1}\left(V_{0}\right), \text { si } k \neq n \\
\text { et } \sum_{F \in \mathcal{F}_{n}} \gamma(F, V) \cdot \Theta_{n}\left(F_{0}\right)=\sigma_{n}(V)=\Theta_{n}\left(V_{0}\right) .
\end{gathered}
$$

Par le théorème A.5, on en déduit :

$$
\left(\begin{array}{c}
\Lambda_{1}^{\mathrm{loc}}(V) \\
\vdots \\
\Lambda_{n}^{\operatorname{loc}}(V)
\end{array}\right)=\left(\begin{array}{ccccc}
m_{1}^{1} & m_{1}^{2} & \ldots & m_{1}^{n-1} & m_{1}^{n} \\
0 & m_{2}^{2} & \ldots & m_{2}^{n-1} & m_{2}^{n} \\
\vdots & & & & \vdots \\
0 & 0 & \ldots & 0 & m_{n}^{n}
\end{array}\right) \cdot\left(\begin{array}{c}
\sigma_{1}(V) \\
\vdots \\
\sigma_{n}(V)
\end{array}\right)
$$

avec :

$$
m_{i}^{i}=a_{i}^{i}=1, m_{i}^{j}=a_{i}^{j}-a_{i}^{j-1}=\frac{\alpha_{j}}{\alpha_{j-i} \cdot \alpha_{i}} C_{j}^{i}-\frac{\alpha_{j-1}}{\alpha_{j-1-i} \cdot \alpha_{i}} C_{j-1}^{i}, \quad \text { si } i+1 \leq j \leq n
$$




\section{Remerciements}

Le premier auteur remercie entre autres A. Bernig, L. Bröker et N. Dutertre pour les nombreuses discussions que nous avons eues lors des rencontres organisées dans le cadre du réseau européen RAAG. Cet article a bénéficié de séjours à l'Institut Max-Planck de Bonn et à l'Institut Weizmann.

\section{RÉFÉRENCES}

[1] A. Bernig, L. Bröcker, Lipschitz-Killing invariants, Math. Nachr. 245 (2002), 5-25.

[2] A. Bernig, L. Bröcker, Courbures intrinsèques dans les catégories analyticogéométriques, Ann. Inst. Fourier (Grenoble) 53 (2003), 1897-1924.

[3] W. Blaschke, Vorlesungen über Integralgeometrie, $3^{\mathrm{e}}$ éd., Deutscher Verlag der Wissenschaften, 1955.

[4] V. G. Boltianskil̆, Hilbert's third problem, John Wiley \& Sons, 1978.

[5] J. BRiançon, J.-P. SPEder, Les conditions de Whitney impliquent $\left(\mu_{*}\right)$ constant, Ann. Inst. Fourier (Grenoble) 26 (1976), 153-163.

[6] L. Bröcker, M. Kuppe, Integral geometry of tame sets, Geom. Dedicata 82 (2000), 285-323.

[7] H. Brodersen, D. Trotman, Whitney (b)-regularity is weaker than Kuo's ratio test for real algebraic stratifications, Math. Scand. 45 (1979), 27-34.

[8] J.-L. Brylinski, A. S. Dubson, M. Kashiwara, Formule de l'indice pour modules holonomes et obstruction d'Euler locale, C. R. Acad. Sci. Paris Sér. I Math. 293 (1981), 573-576.

[9] J. Cheeger, W. Müller, R. Schrader, Kinematic and tube formulas for piecewise linear spaces, Indiana Univ. Math. J. 35 (1986), 737-754.

[10] G. Comte, Formule de Cauchy-Crofton pour la densité des ensembles sousanalytiques, C. R. Acad. Sci. Paris Sér. I Math. 328 (1999), 505-508.

[11] G. Cомте, Équisingularité réelle : nombres de Lelong et images polaires, Ann. Sci. École Norm. Sup. 33 (2000), 757-788.

[12] G. Comte, J.-M. Lion, J.-P. Rolin, Nature log-analytique du volume des sousanalytiques, Illinois J. Math. 44 (2000), 884-888.

[13] R. N. Draper, Intersection theory in analytic geometry, Math. Ann. 180 (1969), 175204.

[14] L. V. D. DRIES, Tame topology and o-minimal structures, London Mathematical Society Lecture Note Series 248, Cambridge University Press, 1998.

$4^{\mathrm{e}}$ SÉRIE - TOME $41-2008-\mathrm{N}^{\mathrm{o}} 2$ 
[15] L. V. D. Dries, C. Miller, Geometric categories and o-minimal structures, Duke Math. J. 84 (1996), 497-540.

[16] A. S. Dubson, Classes caractéristiques des variétés singulières, C. R. Acad. Sci. Paris Sér. $A-B 287$ (1978), A237-A240.

[17] A. S. Dubson, Calcul des invariants numériques des singularités et des applications, Thèse, Unversität Bonn, 1981.

[18] H. Federer, The $(\varphi, k)$ rectifiable subsets of $n$-space, Trans. Amer. Soc. 62 (1947), 114 192.

[19] H. Federer, Curvature measures, Trans. Amer. Math. Soc. 93 (1959), 418-491.

[20] H. Federer, Geometric measure theory, Die Grund. Math. Wiss., Band 153, Springer New York Inc., New York, 1969.

[21] J. H. G. Fu, Tubular neighborhoods in Euclidean spaces, Duke Math. J. 52 (1985), 1025-1046.

[22] J. H. G. Fu, Curvature measures and generalized Morse theory, J. Differential Geom. 30 (1989), 619-642.

[23] J. H. G. Fu, Kinematic formulas in integral geometry, Indiana Univ. Math. J. 39 (1990), 1115-1154.

[24] J. H. G. Fu, Curvature of singular spaces via the normal cycle, in Differential geometry: geometry in mathematical physics and related topics (Los Angeles, CA, 1990), Proc. Sympos. Pure Math. 54, Amer. Math. Soc., 1993, 211-221.

[25] J. H. G. Fu, Curvature measures of subanalytic sets, Amer. J. Math. 116 (1994), 819880.

[26] M. Goresky, R. MacPherson, Stratified Morse theory, Ergebnisse der Mathematik und ihrer Grenzgebiete (3) 14, Springer, 1988.

[27] P. M. Gruber, R. Schneider, Problems in geometric convexity, in Contributions to geometry (Proc. Geom. Sympos., Siegen, 1978), Birkhäuser, 1979, 255-278.

[28] H. Hadwiger, Vorlesungen über Inhalt, Oberfläche und Isoperimetrie, Springer, 1957.

[29] R. M. HARDT, Stratification of real analytic mappings and images, Invent. Math. 28 (1975), 193-208.

[30] R. M. Hardt, Semi-algebraic local-triviality in semi-algebraic mappings, Amer. $J$. Math. 102 (1980), 291-302.

[31] J.-P. Henry, M. Merle, Limites de normales, conditions de Whitney et éclatement d'Hironaka, in Singularities, Part 1 (Arcata, Calif., 1981), Proc. Sympos. Pure Math. 40, Amer. Math. Soc., 1983, 575-584.

[32] J.-P. Henry, M. Merle, Conditions de régularité et éclatements, Ann. Inst. Fourier (Grenoble) 37 (1987), 159-190.

[33] J.-P. Henry, M. Merle, C. Sabbah, Sur la condition de Thom stricte pour un morphisme analytique complexe, Ann. Sci. École Norm. Sup. 17 (1984), 227-268.

[34] H. Hironaka, Normal cones in analytic Whitney stratifications, Publ. Math. I.H.E.S. 36 (1969), 127-138. 
[35] H. Hironaka, Subanalytic sets, in Number theory, algebraic geometry and commutative algebra, in honor of Yasuo Akizuki, Kinokuniya, 1973, 453-493.

[36] H. Hironaka, Stratification and flatness, in Real and complex singularities (Proc. Ninth Nordic Summer School/NAVF Sympos. Math., Oslo, 1976), Sijthoff and Noordhoff, 1977, 199-265.

[37] M. Kashiwara, Index theorem for a maximally overdetermined system of linear differential equations, Proc. Japan Acad. 49 (1973), 803-804.

[38] M. Kashiwara, $B$-functions and holonomic systems. Rationality of roots of $B$-functions, Invent. Math. 38 (1976/77), 33-53.

[39] D. A. Klain, A short proof of Hadwiger's characterization theorem, Mathematika 42 (1995), 329-339.

[40] J. F. Knight, A. Pillay, C. Steinhorn, Definable sets in ordered structures II, Trans. Amer. Math. Soc. 295 (1986), 593-605.

[41] T.-C. Kuo, The ratio test for analytic Whitney stratifications, in Proceedings of Liverpool Singularities-Symposium, I (1969/70), Lecture Notes in Math., Vol. 192, Springer, 1971, 141-149.

[42] M. Kuppe, Integralgeometrie Whitney-stratifizierbarer Mengen, Thèse, Universität Münster, 1999.

[43] K. Kurdyka, J.-B. Poly, G. Raby, Moyennes des fonctions sous-analytiques, densité, cône tangent et tranches, in Real analytic and algebraic geometry (Trento, 1988), Lecture Notes in Math. 1420, Springer, 1990, 170-177.

[44] K. Kurdyka, G. Raby, Densité des ensembles sous-analytiques, Ann. Inst. Fourier (Grenoble) 39 (1989), 753-771.

[45] J. Lafontaine, Mesures de courbure des variétés lisses et des polyèdres (d'après Cheeger-Müller et Schröder), Séminaire Bourbaki, Vol. 1985/86, Exp. n 664, Astérisque 145-146 (1987), 241-256.

[46] R. Langevin, Introduction to integral geometry, $21^{\circ}$ Colóquio Brasileiro de Matemática, Instituto de Matemática Pura e Aplicada (IMPA), Rio de Janeiro, 1997.

[47] R. LANGevin, La petite musique de la géométrie intégrale, in La recherche de la vérité, Écrit. Math., ACL-Éd. Kangourou, Paris, 1999, 117-143.

[48] R. Langevin, T. Shifrin, Polar varieties and integral geometry, Amer. J. Math. 104 (1982), 553-605.

[49] P. Lelong, Intégration sur un ensemble analytique complexe, Bull. Soc. Math. France 85 (1957), 239-262.

[50] J.-M. Lion, Densité des ensembles semi-pfaffiens, Ann. Fac. Sci. Toulouse Math. 7 (1998), 87-92.

[51] J.-M. Lion, J.-P. Rolin, Intégration des fonctions sous-analytiques et volumes des sous-ensembles sous-analytiques, Ann. Inst. Fourier (Grenoble) 48 (1998), 755-767.

[52] T. L. LoI, Verdier and strict Thom stratifications in o-minimal structures, Illinois $J$. Math. 42 (1998), 347-356.

$4^{\mathrm{e}}$ SÉRIE - TOME $41-2008-\mathrm{N}^{\mathrm{o}} 2$ 
[53] R. MacPherson, Chern classes for singular algebraic varieties, Ann. of Math. 100 (1974), 423-432.

[54] J. Mather, Notes on topological stability, Harvard University, 1970.

[55] P. McMullen, R. Schneider, Valuations on convex bodies, in Convexity and its applications, Birkhäuser, 1983, 170-247.

[56] M. Merle, Variétés polaires, stratifications de Whitney et classes de Chern des espaces analytiques complexes (d'après Lê-Teissier), Séminaire Bourbaki, Vol. 1982/83, Exp. $\mathrm{n}^{\circ}$ 600, Astérisque 105 (1983), 65-78.

[57] V. Navarro Aznar, Conditions de Whitney et sections planes, Invent. Math. 61 (1980), 199-225.

[58] V. Navarro Aznar, Stratifications régulières et variétés polaires locales, manuscrit, 1981.

[59] V. Navarro Aznar, D. Trotman, Whitney regularity and generic wings, Ann. Inst. Fourier (Grenoble) 31 (1981), 87-111.

[60] P. Orro, Conditions de régularité, espaces tangents et fonctions de Morse, Thèse, Université d'Orsay, 1984.

[61] P. Orro, D. Trotman, On the regular stratifications and conormal structure of subanalytic sets, Bull. London Math. Soc. 18 (1986), 185-191.

[62] A. Pillay, C. Steinhorn, Definable sets in ordered structures. I, Trans. Amer. Math. Soc. 295 (1986), 565-592.

[63] C. H. SAH, Hilbert's third problem : scissors congruence, Research Notes in Math. 33, Pitman (Advanced Publishing Program), 1979.

[64] L. A. Santaló, Integral geometry and geometric probability, Encyclopedia of Mathematics and its Applications, Vol. 1, Addison-Wesley, 1976.

[65] W. Schicknoff, Whitneysche Tangentenkegel, Multiplizitätsverhalten, NormalPseudoflachheit und Äquisingularitätstheorie für Ramissche Räume, Schr. Math. Inst. Univ. Münster Heft 12 (1977).

[66] R. Schneider, Curvature measures of convex bodies, Ann. Mat. Pura Appl. 116(1978), 101-134.

[67] R. Schneider, A uniqueness theorem for finitely additive invariant measures on a compact homogeneous space, Rend. Circ. Mat. Palermo 30 (1981), 341-344.

[68] R. Schneider, Integral geometry - Measure theoretic approach and stochastic applications, Advanced course on integral geometry, CRM, 1984.

[69] R. SchneIder, Convex bodies : the Brunn-Minkowski theory, Encyclopedia of Mathematics and its Applications 44, Cambridge University Press, 1993.

[70] M. Shiota, Geometry of subanalytic and semialgebraic sets, Progress in Mathematics 150, Birkhäuser, 1997.

[71] J. SteIner, Über parallele Flächen, Monatsber. Preuß. Akad. Wissen. Berlin (1840), 114-118, Ges. Werke, vol. 2 (1882), Reimer, Berlin, 171-176. 
[72] J. Steiner, Von dem Krümmungsschwerpunkte ebener Curven, J. reine angew. Mathematik 21 (1840), 33-63, Ges. Werke, vol. 2 (1882), Reimer, Berlin, 99-159.

[73] B. Teissier, Cycles évanescents, sections planes et conditions de Whitney, in Singularités à Cargèse (Rencontre Singularités Géom. Anal., Inst. Études Sci., Cargèse, 1972), Astérisque, 7-8, Soc. Math. France, 1973, 285-362.

[74] B. Teissier, Variétés polaires II. Multiplicités polaires, sections planes, et conditions de Whitney, in Algebraic geometry (La Rábida, 1981), Lecture Notes in Math. 961, Springer, 1982, 314-491.

[75] R. Thом, Ensembles et morphismes stratifiés, Bull. Amer. Math. Soc. 75 (1969), 240284.

[76] L. D. Tráng, B. Teissier, Variétés polaires locales et classes de Chern des variétés singulières, Ann. of Math. 114 (1981), 457-491.

[77] L. D. TrÁng, B. Teissier, Errata : "Local polar varieties and Chern classes of singular varieties", Ann. of Math. 115 (1982), 668.

[78] L. D. TrÁng, B. Teissier, Cycles evanescents, sections planes et conditions de Whitney II, in Singularities, Part 2 (Arcata, Calif., 1981), Proc. Sympos. Pure Math. 40, Amer. Math. Soc., 1983, 65-103.

[79] D. Trotman, Counterexamples in stratification theory : two discordant horns, in Real and complex singularities (Proc. Ninth Nordic Summer School/NAVF Sympos. Math., Oslo, 1976), Sijthoff and Noordhoff, 1977, 679-686.

[80] D. Trotman, Comparing regularity conditions on stratifications, in Singularities, Part 2 (Arcata, Calif., 1981), Proc. Sympos. Pure Math. 40, Amer. Math. Soc., 1983, 575-586.

[81] G. Valette, Détermination et stabilité du type métrique des singularités, Thèse, Université de Provence, 2003.

[82] G. Valette, Volume, density and Whitney conditions, à paraître dans Ann. Pol. Math. .

[83] J.-L. Verdier, Stratifications de Whitney et théorème de Bertini-Sard, Invent. Math. 36 (1976), 295-312.

[84] H. Weyl, On the Volume of Tubes, Amer. J. Math. 61 (1939), 461-472.

[85] O. Zariski, Studies in equisingularity. I. Equivalent singularities of plane algebroid curves, Amer. J. Math. 87 (1965), 507-536.

[86] O. ZARISKi, Studies in equisingularity II. Equisingularity in codimension 1 (and characteristic zero), Amer. J. Math. 87 (1965), 972-1006.

[87] O. ZARISKI, Studies in equisingularity III. Saturation of local rings and equisingularity, Amer. J. Math. 90 (1968), 961-1023.

[88] O. Zariski, Some open questions in the theory of singularities, Bull. Amer. Math. Soc. 77 (1971), 481-491.

[89] O. Zariski, On equimultiple subvarieties of algebroid hypersurfaces, Proc. Nat. Acad. Sci. U.S.A. 72 (1975), 1425-1426, Correction : Proc. Nat. Acad. Sci. U.S.A. 72 (1975), 3260.

$4^{\mathrm{e}}$ SÉRIE - TOME $41-2008-\mathrm{N}^{\mathrm{o}} 2$ 
[90] O. ZARISKI, Foundations of a general theory of equisingularity on $r$-dimensional algebroid and algebraic varieties, of embedding dimension $r+1$, Amer. J. Math 101 (1979), 453-514.

(Manuscrit reçu le 29 juin 2007 ; accepté, après révision, le 21 janvier 2008.)

\author{
Georges Comte \\ Laboratoire A. Dieudonné, UMR CNRS 6621 \\ Université de Nice, Sophia-Antipolis \\ 28 avenue de Valrose \\ 06108 Nice Cedex 2, France \\ E-mail: Georges.Comte@unice.fr \\ Philippe Graftieaux \\ Laboratoire A. Dieudonné, UMR CNRS 6621 \\ Université de Nice, Sophia-Antipolis \\ 28 avenue de Valrose \\ 06108 Nice Cedex 2, France \\ E-mail: Philippe.Graftieaux@unice.fr \\ Michel Merle \\ Laboratoire A. Dieudonné, UMR CNRS 6621 \\ Université de Nice, Sophia-Antipolis \\ 28 avenue de Valrose \\ 06108 Nice Cedex 2, France \\ E-mail: Michel.Merle@unice.fr
}

